\title{
SUBSURFACE STRUCTURE \& MORPHOLOGY: EPITAXIAL METAL FILM GROWTH STUDIED BY IN SITU X-RAY SCATTERING
}

A Dissertation presented to

the Faculty of the Graduate School

at the University of Missouri

In Partial Fulfillment

of the Requirements for the Degree

Doctor of Philosophy

by

SHAWN T. HAYDEN

Dr. Paul Miceli, Dissertation Supervisor

December 2014 
The undersigned, appointed by the Dean of the Graduate School, have examined the dissertation entitled:

\section{SUBSURFACE STRUCTURE \& MORPHOLOGY: EPITAXIAL METAL FILM GROWTH STUDIED \\ BY IN SITU X-RAY SCATTERING}

presented by Shawn T. Hayden, a candidate for the degree of Doctor of Philosophy and hereby certify that, in their opinion, it is worthy of acceptance.

\begin{tabular}{c}
\hline Dr. Paul Miceli \\
\hline Dr. Suchi Guha \\
\hline Dr. Sashi Satpathy \\
\hline Dr. Gavin King \\
\hline Dr. Robert Winholtz
\end{tabular}

Dr. Linda Godwin 


\section{ACKNOWLEDGMENTS}

I want to thank and acknowledge my wife, Dora, and my children, Crystal, Katie, Shawn Jr., and James. Without your support and love, none of this would be possible.

I wish to express my gratitude to my advisor, Dr. Paul F. Miceli, for his support, guidance throughout this project, and encouragement to keep me on course.

Thank you to the entire Physics and Astronomy Department. I have truly enjoyed

being a part of this department: instructing, TA'ing, and most importantly, having the opportunity to do experimental research. 


\section{TABLE OF CONTENTS}

ACKNOWLEDGMENTS .................. ii

LIST OF TABLES ..................... vi

LIST OF FIGURES .................... vii

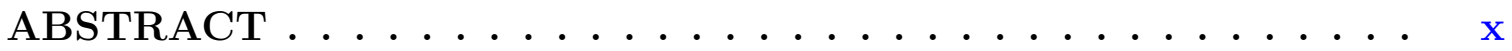

\section{CHAPTER}

1 Introduction ........................ 1

2 Background I: Basic Concepts . . . . . . . . . . . . 6

2.1 Overview ............................... 6

2.2 Thin film growth mechanisms . . . . . . . . . . . . 6

2.2.1 Shadowing: oblique deposition angle . . . . . . . . . . . 8

2.3 Growth modes: surface morphology . . . . . . . . . . . . . . 8

2.3.1 Layer-by-Layer growth . . . . . . . . . . . . . . . . 8

2.3.2 Multilayer growth . . . . . . . . . . . . . . . . 9

2.3.3 Step-flow growth . . . . . . . . . . . . . . . 10

2.4 Point defects . . . . . . . . . . . . . . . . . . . . 10

2.5 Vacancies and downward funneling . . . . . . . . . . . . . . 11

2.6 Film strain . . . . . . . . . . . . . . . . . . . . . 12

3 Background II: Prior Work . . . . . . . . . . . . . . 14

3.1 Vacancies: noble metal homoepitaxy thin film strain . . . . . . . . . . 14 
3.2 Surface morphology: $\mathrm{Cu} / \mathrm{Cu}(100)$ molecular dynamic simulations . . 17

3.3 Vacancy clusters: $\mathrm{Ag} / \mathrm{Ag}(100) \mathrm{X}$-ray diffuse scattering . . . . . . . 19

3.4 Missing material below exposed surface: Glancing Angle Deposition

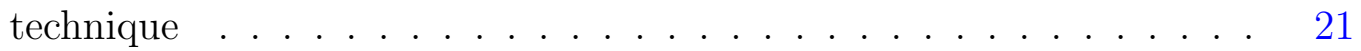

3.5 Conclusion . . . . . . . . . . . . . . . . . . 22

4 Deposition Angle-Dependent Metal Film Growth . . . . . . . . . 24

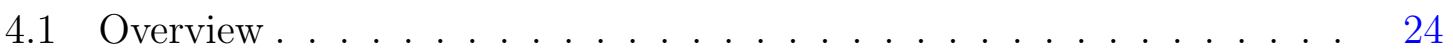

$4.2 \mathrm{X}$-ray reflectivity . . . . . . . . . . . . . 26

4.3 Lower interfacial density of $\mathrm{Ag} / \mathrm{Si}(111) 7 \mathrm{x} 7 \ldots \ldots . . . \ldots 28$

4.3.1 Ag/Si experimental details . . . . . . . . . . . . . . 28

4.3.2 X-ray reflectivity results: Overview . . . . . . . . . . . 30

4.3.3 Scattering Length Density Model . . . . . . . . . . . . . . . . 32

4.3.4 $\Theta_{D}=30^{\circ} \ldots \ldots \ldots \ldots . \ldots \ldots$

4.3.5 $\Theta_{D}=80^{\circ} \ldots \ldots \ldots \ldots$. . . . . . . . . . . . . 39

4.3.6 Exposed surface fraction model: comparison of X-ray reflectivity and AFM measurements . . . . . . . . . . . . 43

4.3.7 X-ray reflectivity and AFM Quantitative Comparison . . . . . 44

4.3 .8 Discussion . . . . . . . . . . . . . . . 46

4.3 .9 Conclusion . . . . . . . . . . . . . . . . . 47

$4.4 \mathrm{Ag}(001)$ homoepitaxial growth . . . . . . . . . . . . . . . 49

4.4.1 Ag/Ag experimental details .............. . . 51

4.4.2 X-ray reflectivity results: Overview . . . . . . . . . . 52

4.4.3 X-ray reflectivity embellished model . . . . . . . . . . . 57 
4.4.4 Incoherent Shadowed Substrate Model . . . . . . . . . . . . . 61

4.4.5 Comparison: incoherent shadowed substrate model with MD simulations ...................... . . 80

4.4.6 Discussion and Conclusion . . . . . . . . . . . . . . . 84

4.5 Conclusions .......................... 85

4.6 Acknowledgements . . . . . . . . . . . . . . . . . 87

A X-ray Reflectivity Homo-Epitaxy Modeling . . . . . . . . 88

A.1 Overview. . . . . . . . . . . . . . . . . 88

A.2 Reflectivity Calculations for 3 layers: $\mathrm{L} 0=\mathrm{sub}, \mathrm{L} 1=$ transition, $\mathrm{L} 2=$ top Overview . . . . . . . . . . . . . . . . . . . 89

A.2.1 Reflectivity Calculations . . . . . . . . . . . . . 90

A.2.2 Averaging . . . . . . . . . . . . . . . . . . 91

A.2.3 $V_{2}^{\prime \prime} \ldots \ldots \ldots \ldots \ldots . \ldots \ldots \ldots$

A.2.4 Conclusion . . . . . . . . . . . . . . . . . . 95

A.3 Abrupt Transistion Fits . . . . . . . . . . . . . . . . . . . . . 96

A.3.1 Conclusion . . . . . . . . . . . . . . . . 96

\section{APPENDIX}

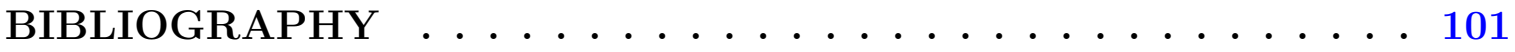

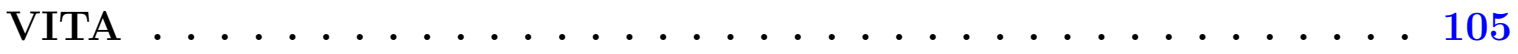




\section{LIST OF TABLES}

Table $\quad$ Page

$4.1 \mathrm{Ag} / \mathrm{Si}(111) 7 \times 7$ best fit reflectivity parameter table $\ldots \ldots \ldots . \ldots 37$

4.2 Structure, Strain, and Disorder Fitting Parameters _ . . . . . . . 81 


\section{LIST OF FIGURES}

Figure

Page

1.1 STM and simulation $\ldots \ldots \ldots \ldots \ldots \ldots \ldots \ldots$

2.1 Thin film growth mechanisms $\ldots \ldots \ldots \ldots \ldots \ldots$

2.2 Shadowing at oblique deposition angle $\ldots \ldots \ldots \ldots$

2.3 Point defects: vacancy defect . . . . . . . . . . . . . . . 11

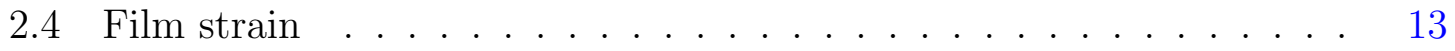

3.1 Noble metal thin film reflectivity $\ldots \ldots \ldots \ldots \ldots$

3.2 Noble metal vacancy mobility . . . . . . . . . . . . 16

3.3 Amar deposition angle dependent morphology evolution . . . . . . . 18

3.4 X-ray diffuse $\mathrm{Ag} / \mathrm{Ag}(001) \ldots \ldots \ldots \ldots \ldots$

4.1 X-ray reflectivity illustration . . . . . . . . . . . . . 27

4.2 X-ray reflectivity measured properties illustrated $\ldots \ldots \ldots 28$

$4.3 \mathrm{Ag} / \mathrm{Si}(111) 7 \times 7 \mathrm{X}$-ray reflectivity measurements $\ldots \ldots \ldots . \ldots 31$

$4.4 \mathrm{Ag} / \mathrm{Si}(111) 7 \times 7$ SLD profile model . . . . . . . . . . . . 34

$4.5 \mathrm{Ag} / \mathrm{Si}(111) 7 \times 7 \rho_{b}(z)$ model with missing material $\ldots \ldots \ldots . \ldots 34$

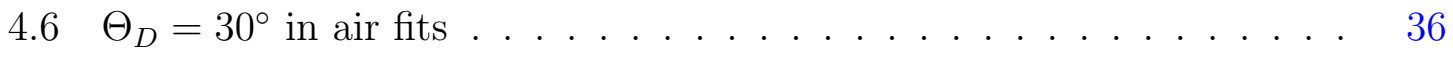




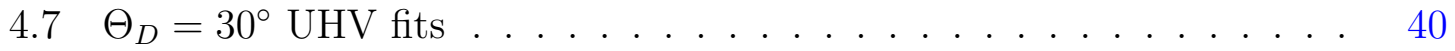

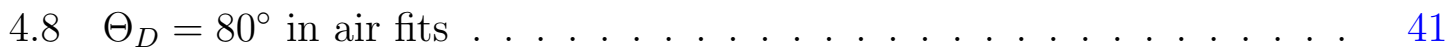

4.9 AFM measurements for sample 80A . . . . . . . . . . . . 42

4.10 Exposed surface fraction schematic $\ldots \ldots \ldots \ldots$

4.11 X-ray reflectivity and AFM Quantitative comparison . . . . . . . 45

4.12 Deposition angle dependent $\rho_{b}$ comparison $\ldots \ldots \ldots \ldots$

4.13 Growth possibilities . . . . . . . . . . . . . . . . . 49

4.14 XRR $\mathrm{Ag} / \mathrm{Ag}(100)$ deposition angle "Pearl Necklace" . . . . . . . . . 50

4.15 APS 6ID-C UHV in situ surface chamber . . . . . . . . . . . 53

4.16 Anti-Bragg intensity vs coverage . . . . . . . . . . 54

4.17 Anti-Bragg intensity vs deposition angle exp. fit . . . . . . 55

4.18 Incoherent scattering from clean surface $\ldots \ldots \ldots \ldots$

4.19 Pyramidal model . . . . . . . . . . . . . . . . . 57

4.20 Embellished model . . . . . . . . . . . . . . . . . 58

4.21 Embellished model fit for $\Theta_{D}=60^{\circ}$ 24ML data $\ldots \ldots \ldots . . . .59$

4.22 Embellished model parameters for 24ML . . . . . . . . . . 60

4.23 Density modeled Profile . . . . . . . . . . . . . . . . . 64

4.24 Strained Lattice Spacing modeled Profile . . . . . . . . . . . . . 64

4.25 Disorder Modeled Profile . . . . . . . . . . . . . . . . . 65

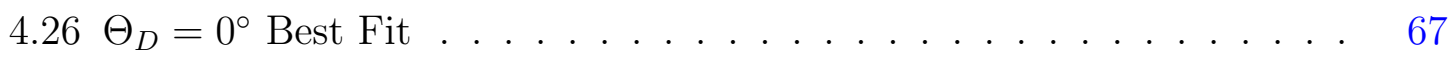

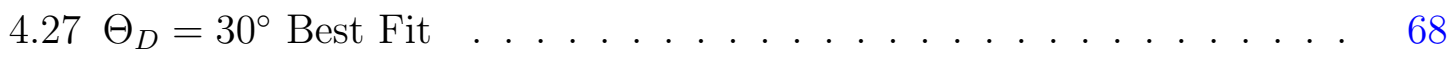

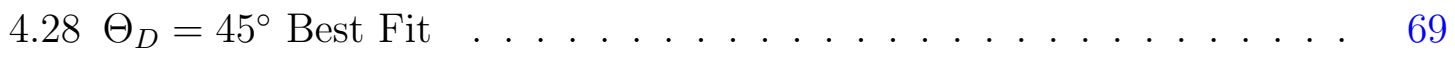

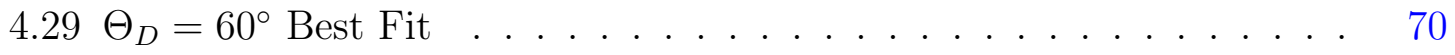

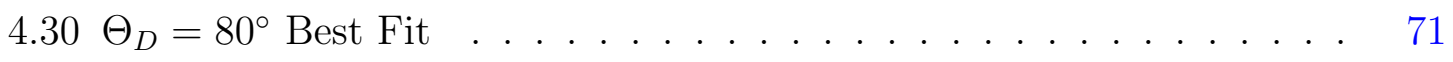


4.31 Structure, Strain, and Disorder Fitting Distributions . . . . . . . 73

$4.32 f_{h}$, clean surface incoherent scattering . . . . . . . . . . . . 74

4.33 Modeled film coverage versus experimental calibrated coverage as a function of deposition angle . . . . . . . . . . . . . . 75

4.34 Modeled film exposed surface roughness . . . . . . . . . . . . 76

4.35 Disorder $\alpha$ linear parameter . . . . . . . . . . . . . . . . 77

$4.36 \delta d_{1}$ vs deposition angle . . . . . . . . . . . . . . . . 78

$4.37 N_{D 1}$ vs deposition angle . . . . . . . . . . . . . . . 79

4.38 Average strain vs coverage . . . . . . . . . . . . . 80

4.39 Surface roughness width Amar comparison . . . . . . . . . . 83

4.40 Average strain Amar comparison . . . . . . . . . . . . . . . . 83

A.1 3 Layer Model . . . . . . . . . . . . . . . . . . . . . . . . 97

A.2 Fits $\Theta_{D}=0^{\circ} 6 \mathrm{ML} \ldots \ldots \ldots \ldots$. . . . . . . . . . . . . 98

A.3 Fits $\Theta_{D}=0^{\circ} 12 \mathrm{ML} \ldots \ldots \ldots \ldots 9$

A.4 Fits $\Theta_{D}=0^{\circ} 24 \mathrm{ML} \ldots \ldots \ldots \ldots$. . . . . . . . . . . 100 


\begin{abstract}
The existence of missing material (vacancies) below the exposed surface was deduced from deposition-angle-dependent experimental evidence.

Due to a lack of experimental evidence, theoretical film growth models have largely neglected the incorporation of vacancies. In fact, downward funneling is a key construct introduced to remove vacancies, therefore, the growth mechanisms for vacancies are not well understood. Vacancies can even explain the anomalous change in physical film properties, such as conductivity[1] and film strain[2].

There are two schools of thought concerning how film strain is incorporated during film growth: 1) structural surface morphology[3] and 2) vacancy clusters[4]. Left out of the discussion is the possible interpretation that there is an interplay between surface morphology and vacancies. In the case of islands growth, surface morphology dominates, and in the smooth film growth case, vacancies could dominate. Is it surface morphology or is it vacancies that are responsible for the measured film strain? This is a complicated question that does not have a direct answer. In order for this question to be addressed, the buried interface has to be characterized.

It will be shown, through the deposition-angle-dependent characterization of the $\mathrm{Ag} / \mathrm{Si}(111) 7 \mathrm{x} 7$ system, using $\mathrm{X}$-ray reflectivity, which is sensitive to the buried interface, that missing material at the interface exists. The characterization of the $\mathrm{Ag} / \mathrm{Ag}(001)$ deposition-angle-dependent "Pearl Necklace" data, using X-ray reflectivity, shows a constant strain below the exposed surface irrespective of the growth conditions: the deposition angle, the coverage, or the surface roughness. This constant strain below the surface, in spite of the growth conditions, is attributed to the buried missing material trapped during film growth.
\end{abstract}




\section{Chapter 1}

\section{Introduction}

For the advancement of basic science, it is critical to understand, predict, and control the fundamental thin film growth processes and mechanisms. Secondly, in commercial applications, it is important to be able to control the growth of thin films, so one can design, adjust, and tune the desired physical properties.

When an atom is missing from its atomic location, it is considered to be a vacancy defect. If there is a large number of missing atoms conglomerated together, then all the missing material together is called a void or a vacancy cluster defect. This missing material can affect the physical properties of the film such as conductivity[1] and film strain[2], which can impact the growth behavior of thin films. Improving the current understanding of thin film growth by studying and modeling basic systems that show interesting growth phenomena is the focus of my research. Of particular interest is missing material buried below the film's exposed surface $[2,4]$ which can come in the form of vacancies, vacancy clusters, overhangs, or voids that are possibly connected to the surface. It will shown that there is significant evidence for missing material below 
the exposed surface that has to be considered to properly in order to understand thin film growth mechanisms.

Elusive subsurface structure (vacancies), beneath the exposed surface of low temperature metal homoepitaxially grown films, has been uncovered experimentally using $\mathrm{X}$-ray diffuse scattering[4]. X-rays are a non-invasive tool which penetrate below the surface and have been useful in uncovering these vacancies. There is a wealth of research in the area of thin film epitaxial growth. Yet the lack of evidence for missing material below the exposed surface has thwarted a proper understanding of the physical growth mechanisms leading to the incorporation of vacancies. Conventional surface probes, such as STM, just look at the surface, while the theory just relies on the information provided by the experimentalist, as shown in Figure 1.1. As a consequence, most theoretical growth models have intentionally removed voids, overhangs, or vacancies through constructs such as downward funneling[5]. Therefore, both theory and experiments have overlooked the significance of large vacancy clusters during film growth.

In a simple noble metal system that was believed to have been well understood, an important growth mechanism responsible for vacancy formation has been overlooked. The $\mathrm{Ag} / \mathrm{Ag}(001)$ low temperature $\mathrm{X}$-ray diffuse scattering measurements showed large vacancy clusters[4] in low concentrations. Now, since missing material has been seen and measured experimentally, it can be used to provide an insight into the interplay of associated atomic-scale growth kinetics, and it should not be intentionally modeled out through the use of geometric constructs like downward funneling. 


\section{STM}
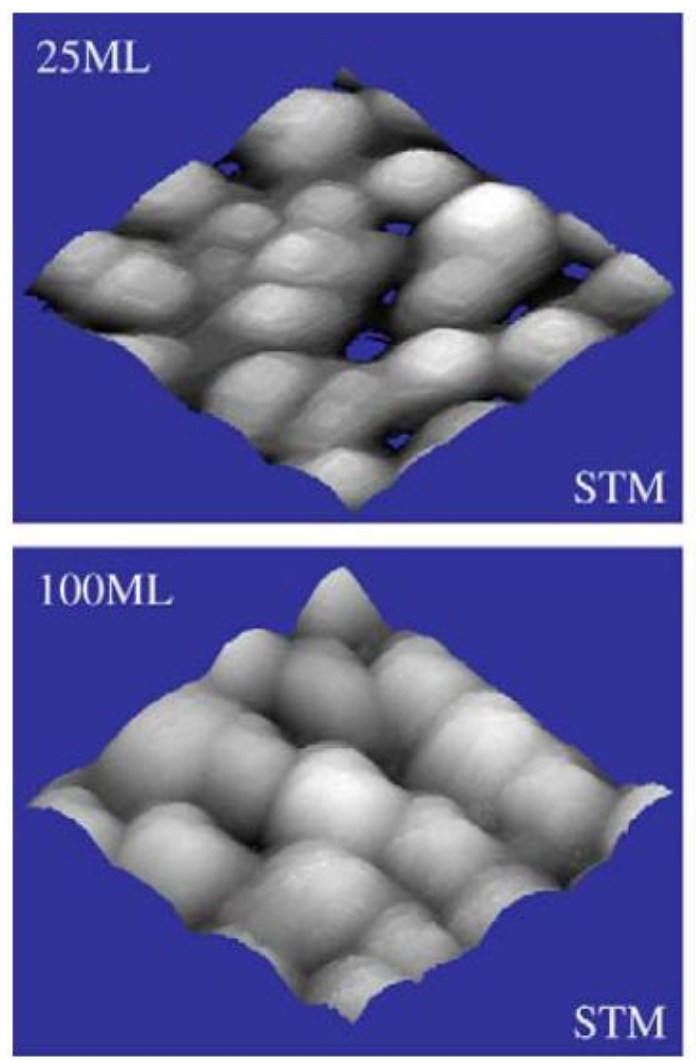

\section{Simulation}
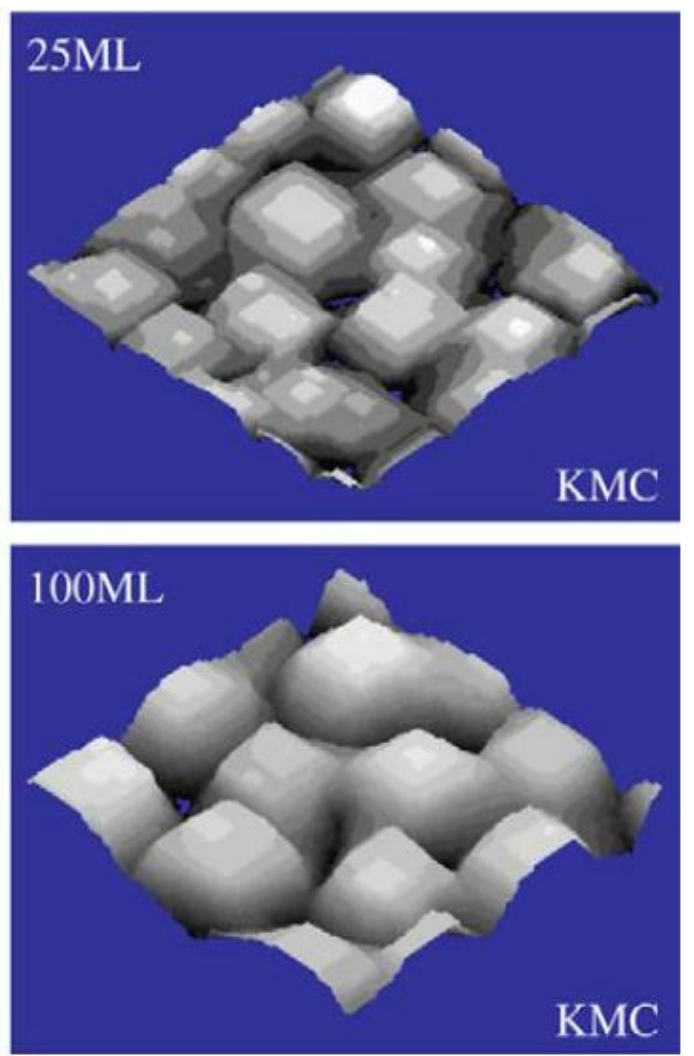

Figure 1.1: Shows on the left a conventional STM surface probe image and on the right are theoretical simulations which used the experimental results given. From Ref. [6].

The physical properties of the film, such as conductivity[1] and film strain[2], can be affected by vacancies. Thin film growth is also a vital component in the fabrication of modern day semiconductor devices as controlling the growth environment allows for the tuning of the film's physical properties to meet functional requirements. Therefore, predicting growth behavior through the understanding of growth kinetics is important for the advancement of modern technology. In addition to the practical applications, the characterization and understanding of thin film growth mechanisms 
is an important subject of basic condensed matter physics research.

Vacancy incorporation has been credited for the thin film surface-normal compressive strain observed in $\mathrm{X}$-ray reflectivity studies on several different morphological systems[2]. There exist two opposing schools of thought when it comes to understanding how this film strain is incorporated during film growth: 1) structural surface morphology[3], and 2) vacancy clusters[4]. Amar's group performed deposition angle dependent molecular dynamics simulations for thin film $\mathrm{Cu} / \mathrm{Cu}(001)$ which attributes the thin-film strain fields to nano-surface structures[3]. However, no large vacancy clusters were observed in these simulations. In contrast, X-ray diffuse measurements[4] on $\mathrm{Ag} / \mathrm{Ag} 100 \mathrm{ML}$ have shown large vacancy clusters buried below the exposed surface which give rise to the strain seen in the film.

Is it surface morphology or is it the vacancies that are responsible for the measured film strain? This is a complicated question with no direct answer at this time. In order to address this question, the buried missing material will be characterized using $\mathrm{X}$-ray reflectivity, for a series of films grown at varying deposition angles and coverages.

Additional experimental evidence for the existence of missing material below the exposed surface for varying deposition angles will be shown. Deposition angle dependent X-ray reflectivity is able to measure a region that is sensitive to this missing material. The characterization of the $\mathrm{Ag} / \mathrm{Si}(111) 7 \mathrm{x} 7$ system using $\mathrm{X}$-ray reflectivity along with AFM confirms missing material. The characterization of missing material in the $\mathrm{Ag} / \mathrm{Ag}(001)$ using $\mathrm{X}$-ray reflectivity as the primary investigation tool, is still in progress.

Chapter 2 provides the necessary background information on epitaxial growth, growth modes, point defects, vacancies and downward funneling, followed by film 
strain in order to understand the subsequent chapters. Chapter 3 motivates the continued study of missing material at the buried film/substrate interface. It provides a historical perspective on research related to the strains caused by vacancies in homoepitaxy. It will be shown that the experimental and theoretical research has not thoroughly addressed the issue, and has left more questions than answers. This perspective provides the necessary ingredients in which the research can be carried out to address the question of strains caused by vacancies. Chapter 4 is the culmination of the work done on the $\mathrm{Ag} / \mathrm{Ag}(001)$ and $\mathrm{Ag} / \mathrm{Si}(111) 7 \times 7$ systems which characterizes the differently grown films and shows evidence for missing material below the exposed surface. Chapter 5 is the conclusion and an overview of my proposed future work. 


\section{Chapter 2}

\section{Background I: Basic Concepts}

\section{$2.1 \quad$ Overview}

This chapter presents background information on epitaxial growth, growth modes, point defects, vacancies and downward funneling, followed by film strain which would be useful to understand the subsequent chapters.

\subsection{Thin film growth mechanisms}

The main atomic processes (Figure 2.1) occurring during epitaxial growth[6] include: a) deposition, b) nucleation on a hollow adsorption site, c) diffusion on a flat terrace, d) aggregation (attachment to an existing island), e) edge diffusion, f) step-ledge diffusion and g) downward funneling. Kinetic active diffusion of atoms is dictated by the substrate temperature. The competition between these events dictates the 


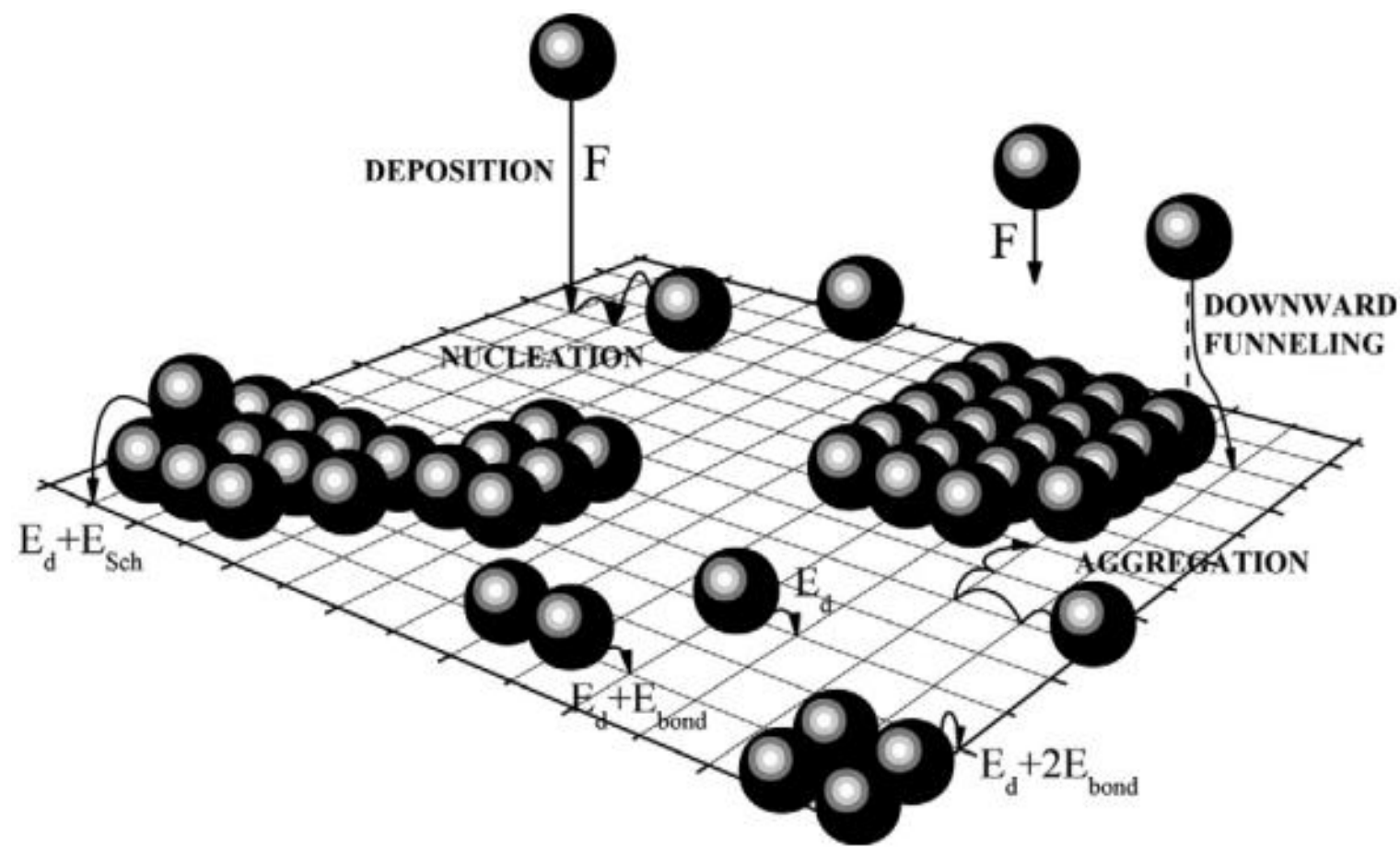

Figure 2.1: Schematic illustrating epitaxial growth mechanisms. From Ref. [6]

resulting surface morphology.

Downward funneling is a geometric smoothing effect in which atoms deposited on step-ledges go to lower adsorption sites[5]. Ehrlich-Schwoebel step-ledge diffusion $[7,8]$ is the additional diffusion barrier encountered by an atom when crossing an atomic ledge from a higher terrace level to a lower terrace level. 


\subsubsection{Shadowing: oblique deposition angle}

Shadowing is a geometrical growth effect in which the depositing atoms hits the side of the growing film nano-structures thus creating a "shadow" where the atoms can not be deposited, preventing valleys from filling in, as illustrated in Figure 2.2. Atomic shadowing can be enhanced by increasing the deposition angle $\left(\Theta_{D}\right)$, measured with respect to the sample normal. These enhancements can lead to rough surfaces and large columnar structure growth $[9,10]$.

\subsection{Growth modes: surface morphology}

There are several characteristic growth modes for homoepitaxial growth: layer-bylayer (LBL), multilayer, and step-flow. Film growth involves the competition between nucleation and diffusion processes for deposited atoms. This competition dictates and influences the development of subsequent film morphology and properties.

\subsubsection{Layer-by-Layer growth}

The nucleation and growth of two-dimensional islands is referred to as layer-by-layer growth (Frank-van der Merwe). A single layer completes before the next layer starts to grow yielding a smooth film. This is a case where the step-ledge barrier is low

compared to the kinetic active diffusion of the deposited atom. $\mathrm{Ag} / \mathrm{Ag}(001)$ is one example of a system that exhibits LBL growth[11]. 


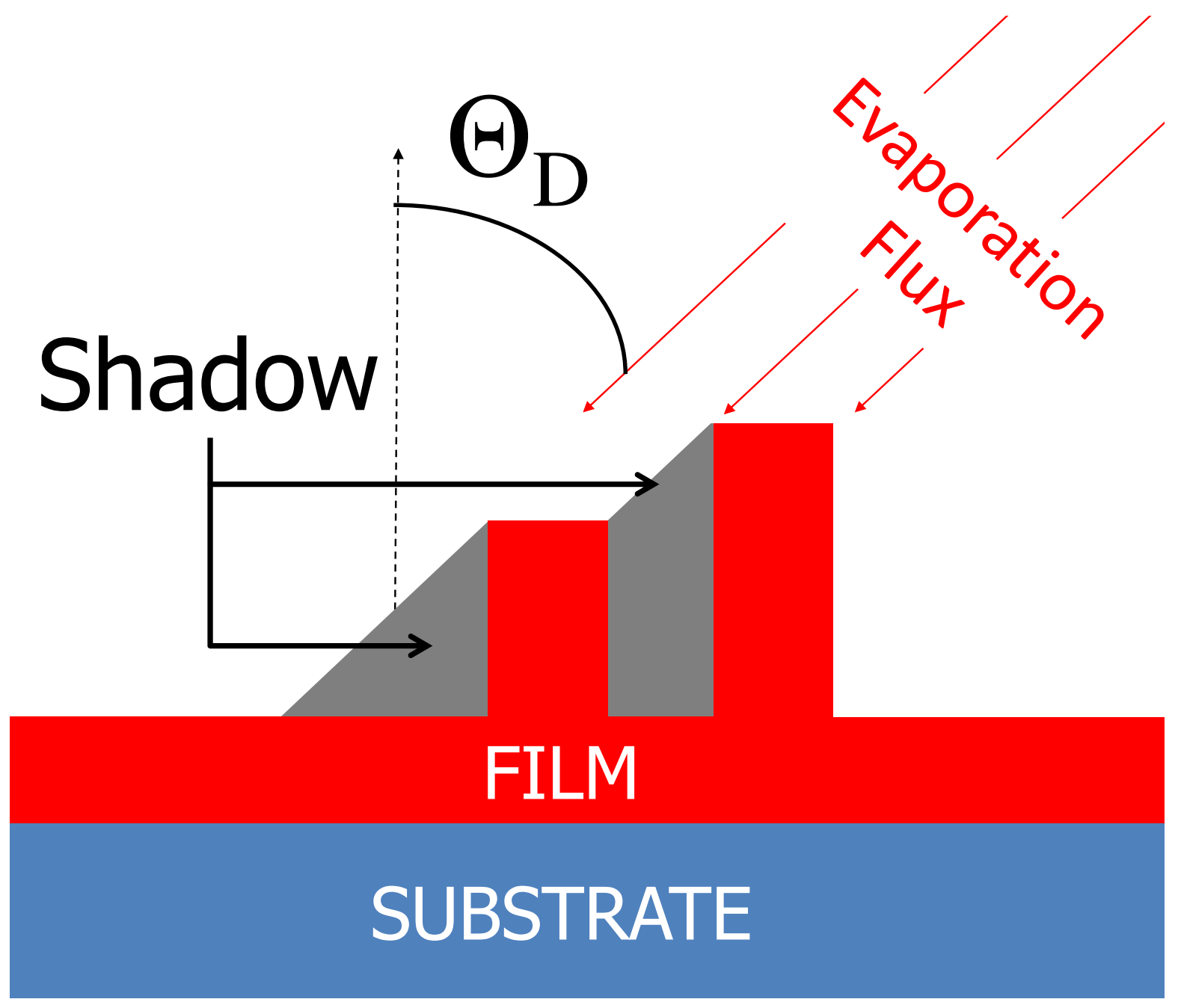

Figure 2.2: Schematic illustrating the geometrical deposition growth of shadowing.

\subsubsection{Multilayer growth}

In multilayer growth (VolmerWeber), three-dimensional clusters (islands) grow on the substrate surface. There is no continuous layer-by-layer growth involved. Also the step-ledge barrier is too high for atoms to go down to the next layer. $\mathrm{Ag} / \mathrm{Ag}(111)$ exhibits multilayer growth[11]. 


\subsubsection{Step-flow growth}

Step-flow growth process occurs when the average terrace width is much smaller than the island separation. It is more likely for deposited atoms to diffuse and aggregate on pre-existing steps which is called step propagation or step-flow. The growing surface is viewed as steps traveling across the surface. These features are common for metal homoepitaxy and have been observed for $\mathrm{Cu}(001)[6]$.

\subsection{Point defects}

It is seldom that a film is perfectly ordered. It is useful to think about the film being constructed in terms of a series of regular stacked planes of atoms. There are four basic mechanisms for introducing a point defect into the film, as illustrated in Figure 2.3 .

- Substitutional impurities occur when an atom type, that is supposed to be there, is replaced with a different type.

- Vacancies occur when an atom is missing from its lattice site.

- Dislocations are one-dimensional defects caused by holes that are not large enough to be considered a vacancy.

- Interstitial impurities are described when an atom forces its way into a hole in between lattice site 


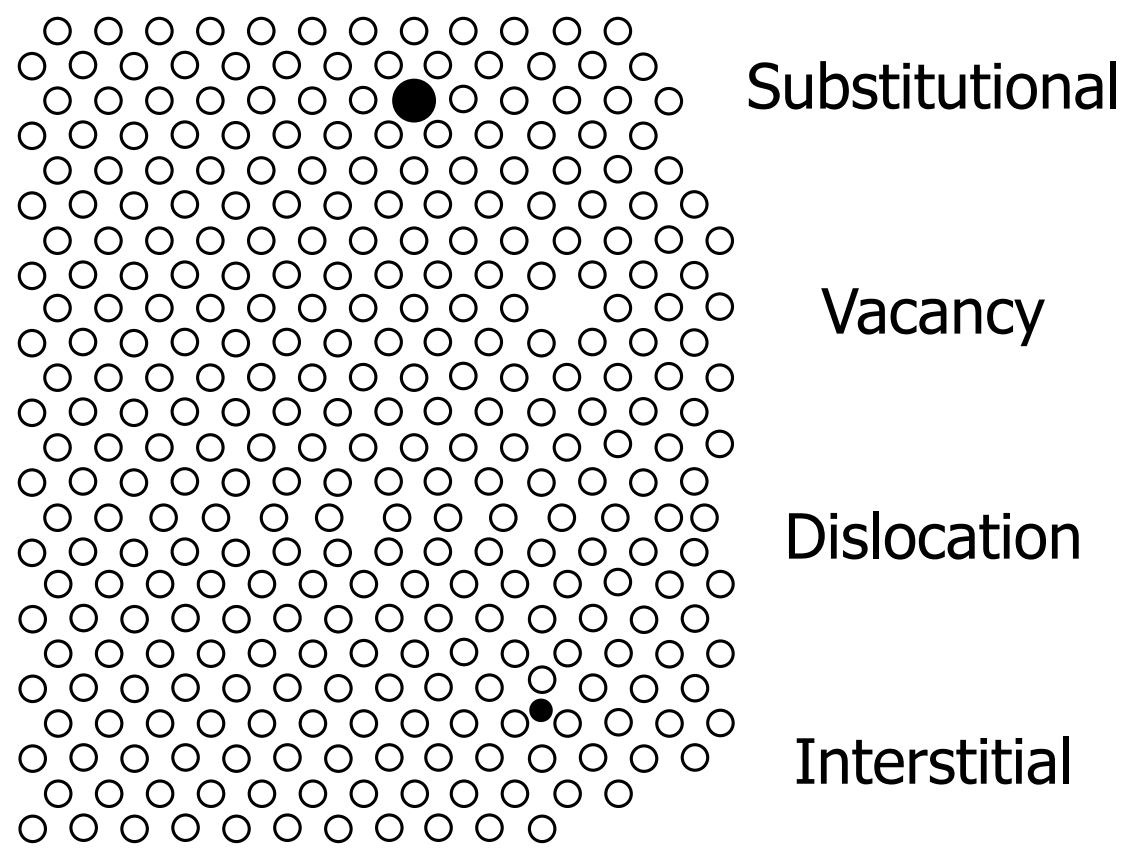

Figure 2.3: Schematic illustrating the different types of geometrical defects.

\subsection{Vacancies and downward funneling}

Due to a lack of experimental evidence, film growth theoretical models have largely neglected the incorporation of vacancies. In fact, downward funneling is a key construct introduced to remove vacancies.

The competition between downward funneling and step-ledge diffusion has been effective in successfully describing quantitatively the surface morphology evolution during homoepitaxial crystal growth[6]. Also, demonstrated by restricting downward funneling, was the reentrant rough growth at low temperatures for $\operatorname{Ag}(001)[12]$. Even though this did enable the formation of vacancies, restricted downward funneling, in the higher temperature range, would lead to surfaces that are much rougher than those observed experimentally and, would therefore be inconsistent with known kinetics. Furthermore, vacancy clusters have been observed at these higher temperatures and 
are quite large as described in Section 3.3. These facts bring to light that the growth mechanisms for vacancy and vacancy cluster formation are not well understood. It is likely that a complex physical process is responsible for vacancy growth, since the downward funneling mechanism is a geometrical construction.

\subsection{Film strain}

Surface-normal film strain is defined as the difference between the film lattice spacing $\left(d_{\text {film }}\right)$ and the substrate lattice spacing $\left(d_{\text {substrate }}\right),\left(d_{\text {film }}-d_{\text {substrate }}\right) / d_{\text {substrate }}$, as illustrated in Figure 2.4. Film strain can occur from:

- multi-layer surface morphology allowing the relaxation of latterly exposed atoms

- lattice parameter mismatch at buried interface normally seen in heteroepitaxy

- vacancy incorporation which would lead to a lattice compression $\left(d_{f i l m}<d_{\text {substrate }}\right)$.

Lattice parameter mismatch can be ruled out for homoepitaxy systems because of the absence of a true buried interface. 


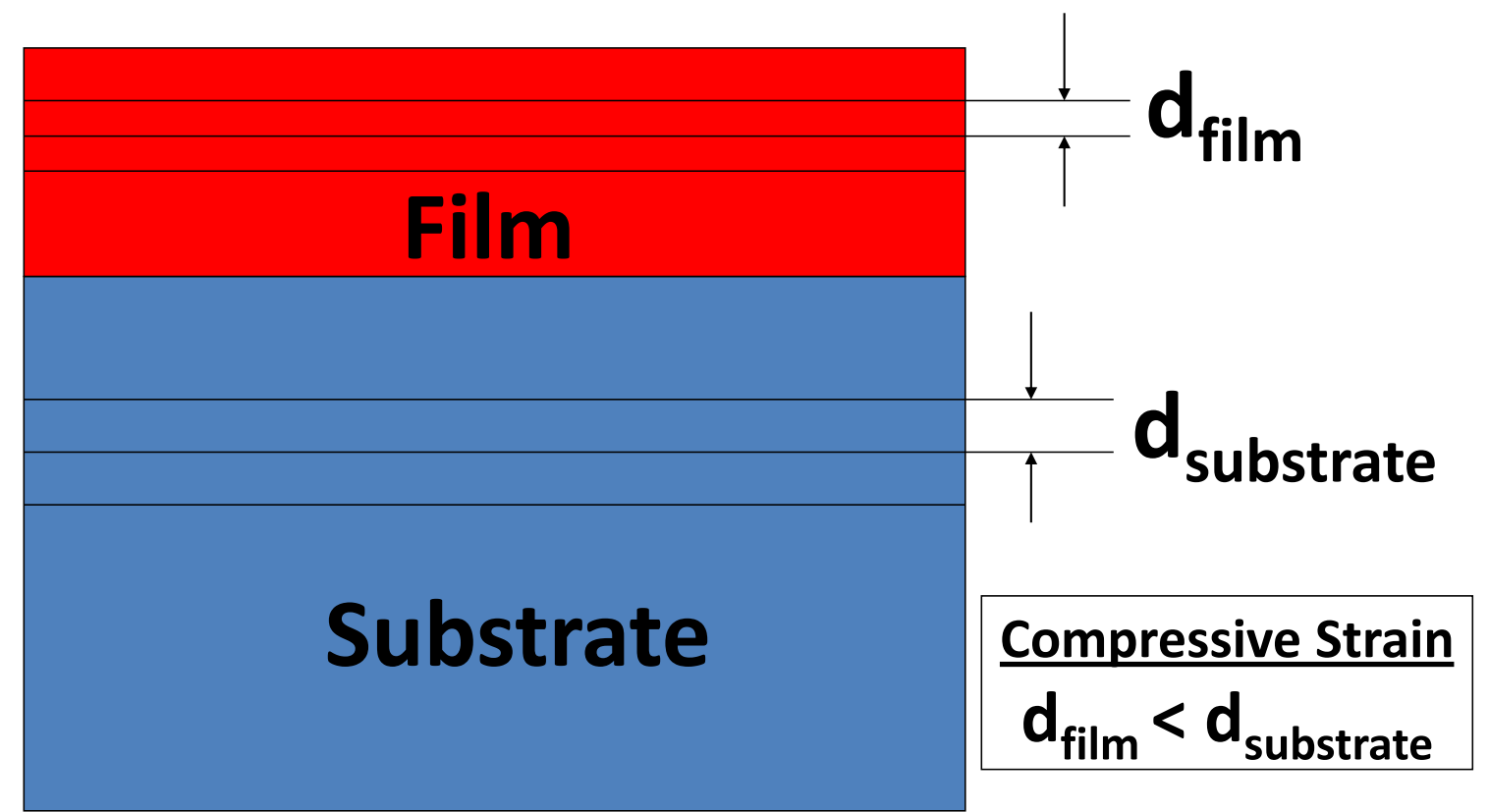

Figure 2.4: Schematic illustrating the strain of a thin film on top of a substrate. The average film lattice parameter is smaller than the average lattice parameter for the bulk. 


\section{Chapter 3}

\section{Background II: Prior Work}

\subsection{Vacancies: noble metal homoepitaxy thin film strain}

Vacancy incorporation has been credited for the thin film surface-normal compressive strain observed in X-ray reflectivity studies[2, 11]. Three different homoepitaxially noble metal systems, all grown at $T \sim 100 \mathrm{~K}, \mathrm{Ag}(111), \mathrm{Cu}(100)$, and $\mathrm{Ag}(100)$, spanned a range of growth modes from multilayer to layer-by-layer surface morphology growth, respectively. A $1 \%$ compressive strain for all three growth systems was observed from the oscillations shown in Figure 3.1. Since the surface morphologies were so drastically different and yet, they all yielded the same strain, it was inferred that vacancies were the only reasonable cause for the strain. Upon annealing the $\mathrm{Cu}$ sample, as shown in Figure 3.2, the observed strain started going to zero at the same temperature as vacancy mobility in irradiated $\mathrm{Cu}[13]$. Thus providing further evidence for vacancies. 


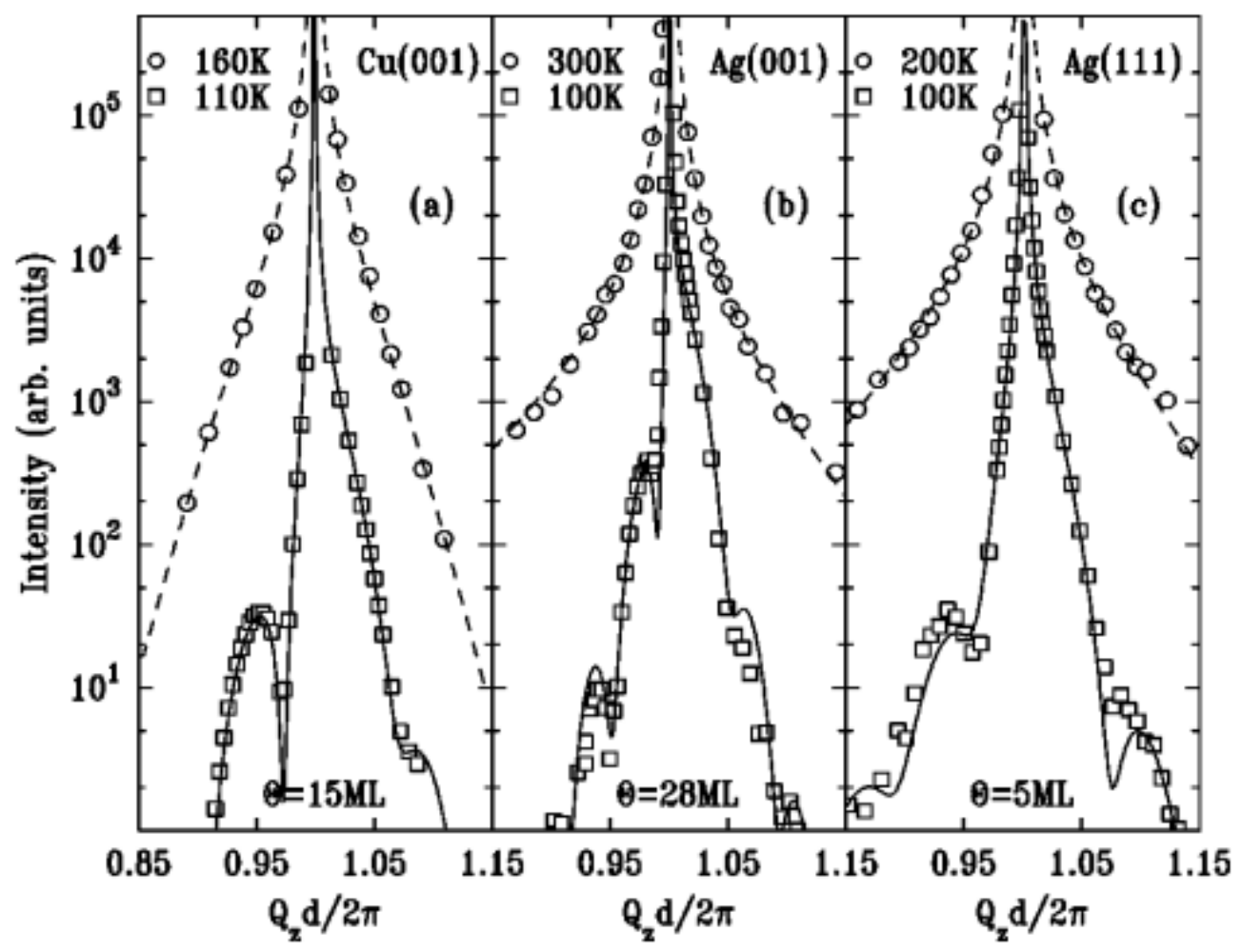

Figure 3.1: X-ray reflectivity for three noble metal systems: left $\mathrm{Cu}(100)$, middle $\operatorname{Ag}(100)$, and right $\operatorname{Ag}(111)$. The thin film oscillations were caused by a compressive strain. From Ref. [2].

All three systems exhibited different types of morphological structural growth. The $\operatorname{Ag}(111)$ system showed multilayer structural growth, the $\operatorname{Ag}(100)$ system exhibited layer-by-layer growth, and the $\mathrm{Cu}$ system morphology was somewhere in between the two Ag systems. Missing from these studies is the strain and morphology dependence as a function of deposition angle growth. At the time of these studies, the deposition angle dependence was not considered.

Island growth (multilayer) should not play a role in the $\mathrm{Ag}(100)$ growth, therefore vacancies could be inferred for the homogeneous compressive strain. Left out was the possible interpretation that there is an interplay between surface morphology 


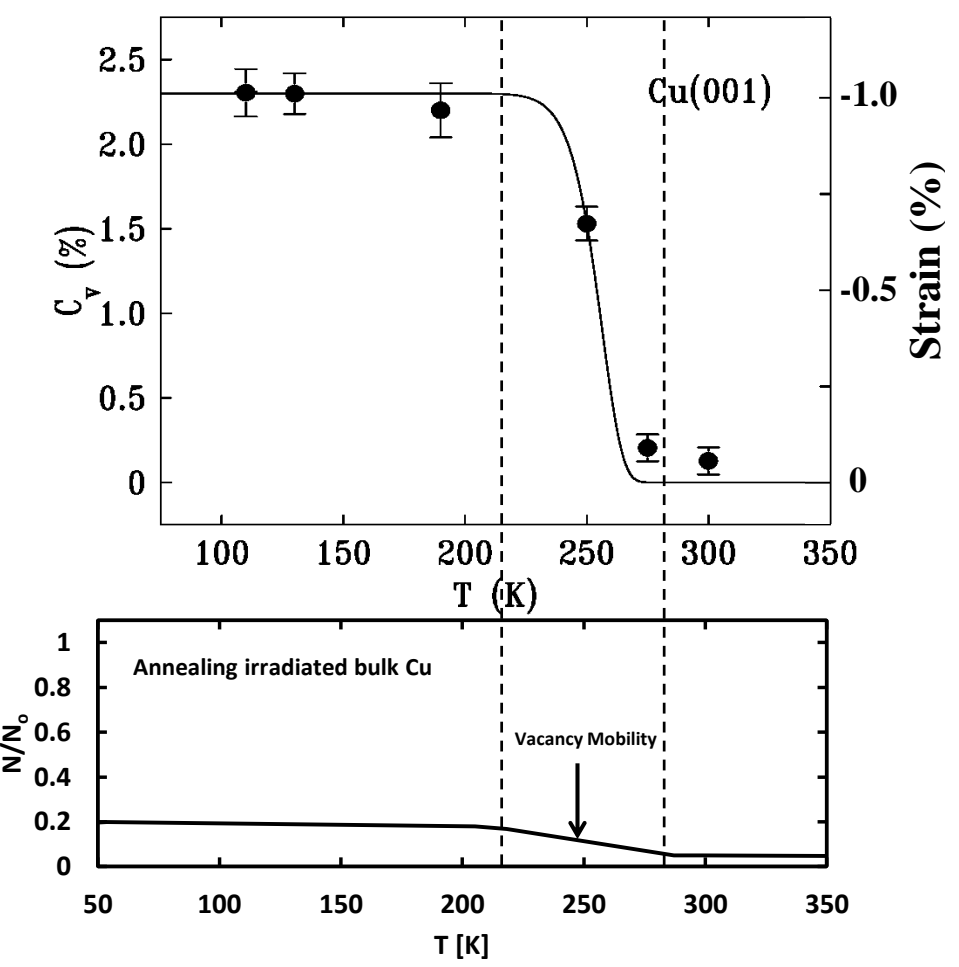

Figure 3.2: Top plot shows the vacancy concentration and the measured strain as a function of annealing temperature. The bottom plot shows vacancy mobility in irradiated $\mathrm{Cu}[?]$ occurring at the same temperature as the vacancy concentration in the top plot starts going to zero. Top figure from Ref. [2].

and vacancies for the different systems that yields the same $1 \%$ strain. The growth mechanism to get the $1 \%$ strain is somehow similar in both cases of multilayer growth and the incorporation of vacancies. If this were true, then the $\operatorname{Ag}(111)$ multilayer system would exhibit a morphological strain that would be equal to the $\operatorname{Ag}(100)$ vacancy induced strain.

Possible experiments to test this idea would be $\mathrm{X}$-ray diffuse measurements as a function of deposition angle on the $\mathrm{Ag} / \mathrm{Ag}(100)$. It would exhibit layer-by-layer growth for normal incidence all the way to multilayer growth for oblique deposition angles. It would be unfortunate if the scattering theory for buried defect clusters 
somehow yielded the same results as the diffuse scattering from multi-layer systems. This idea needs to be explored further. Additional, much easier experiments would be $\mathrm{X}$-ray reflectivity measurements as a function of deposition angle for several coverages on $\mathrm{Ag} / \mathrm{Ag}(100)$ for the same reasons as above. Characterizing the vacancies, surface morphology and strain and looking for correlation would help to determine which effect is dominating the strain.

\subsection{Surface morphology: $\mathrm{Cu} / \mathrm{Cu}(100)$ molecular dynamic simulations}

Another interpretation for the cause of the strain measured on the $\mathrm{Cu} / \mathrm{Cu}(100)$ system comes through the use of molecular dynamics simulations[3]. Several growth environments were simulated as a function of coverage for several deposition angles, from $0^{\circ}$ to $80^{\circ}$. These simulations yielded an average $1 \%$ compressive strain through surface morphology induced relaxation. This means the surface structures shown in Figure 3.3 were responsible for the strain. The hill and valley structures are grown because of the effects of shadowing, hills preventing the valleys from filling in during deposition, combined with downward funneling (DF) suppression, a thermally activated diffusion process.

These simulated $\mathrm{Cu}$ systems were all dominated by multilayer types of surface structure. Taking a close look at the $7 \mathrm{ML} 0^{\circ}$ deposition angle simulation, shown in the top left of Figure 3.3, reveals a significant surface structure. It would then seem reasonable that the simulated systems observed strains would all be dominated by surface morphology and not vacancies. Vacancies were also seen in low concentrations, 


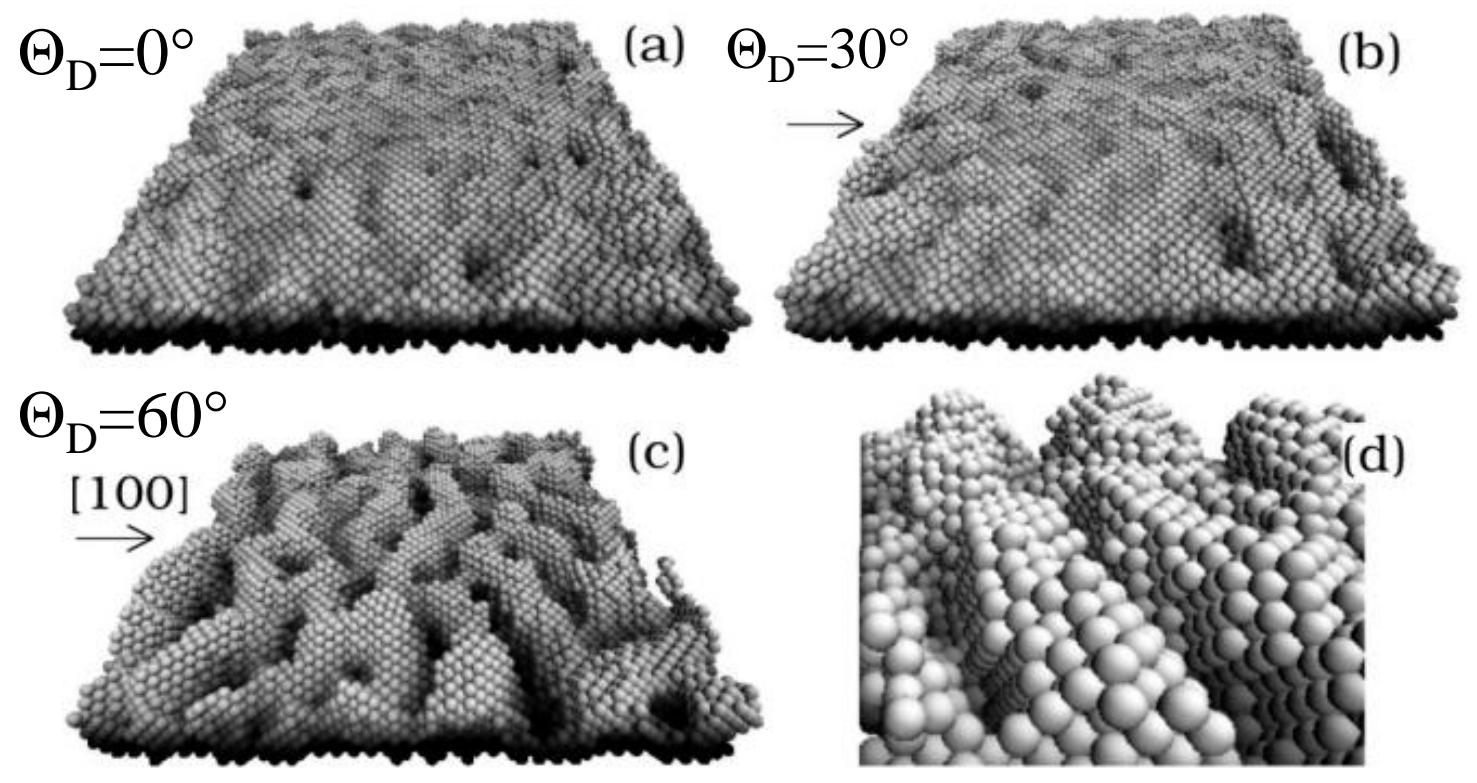

Figure 3.3: $\mathrm{Cu} / \mathrm{Cu}(100) \mathrm{MD}$ simulations showing the surface morphological evolution as a function of deposition angle. Distinct surface structure features are seen as hills and valleys for the $60^{\circ}$ deposition angle. From Ref. [3].

$\sim 0.05 \%$. However, when they were filled in, the system was allowed to relax, and there was no significant change in the compressive strain, as previously expected due to the surface structure already dominating at low coverages and normal deposition angle deposition. Large vacancy clusters, as seen in the X-ray diffuse[4], were not observed in these simulations.

One must be careful when interpreting the MD simulations. No large vacancy clusters were observed in these simulations. The system size is small relative to observed structural features. As will be shown later in Section 4.3.5, the total system size simulated $\left(500 \mathrm{~nm}^{2}\right)$ is smaller than the observed structural length scales of $2500 \mathrm{~nm}^{2}$. Also, missing is the simulation of a system that is dominated by layer-bylayer growth. 
It is still not clear as to the mechanism that causes the compressive strain for systems which exhibit layer-by-layer growth such as $\mathrm{Ag} / \mathrm{Ag}(100)$. It is still possible that there is a competition between the surface morphology, which dominates at large deposition angles, and vacancies, which would dominate at lower deposition angles. For these growth systems, surface structures caused by shadowing effects, such as large hills and valleys, and DF suppression would be missing. This would mean that vacancies were responsible for the observed strain in these systems.

\subsection{Vacancy clusters: $\mathrm{Ag} / \mathrm{Ag}(100) \mathrm{X}$-ray diffuse scattering}

To determine the cause for the strain in the $\mathrm{X}$-ray reflectivity measurements in Section 3.1, X-ray diffuse scattering measurements near Bragg reflections were performed on a layer-by-layer growth system, $\mathrm{Ag} / \mathrm{Ag}(100)[4]$. X-ray diffuse scattering is a useful tool to measure local strain fields buried beneath the surface.

Measurements, on 100ML $\mathrm{Ag} / \mathrm{Ag}(001)$ films, confirmed the existence of vacancy clusters (large volume of missing material). The diffuse model (diffuse scattering from defect clusters[14]), impeccably fit the data over a wide Q range, as shown in Figure 3.4. The average vacancy cluster size was at least $750 \AA^{-3}$ with a concentration of $0.05 \%$ which corresponds to a $1 \%$ compressive strain. This same strain was seen in the previous reflectivity measurements.

Vacancies, in this layer-by-layer system, were shown to be responsible for the observed strain seen in the previous reflectivity measurements (previous work only inferred vacancies). To understand the evolution of the strain contribution from 
$100 \mathrm{ML} \quad$ Subtracted data

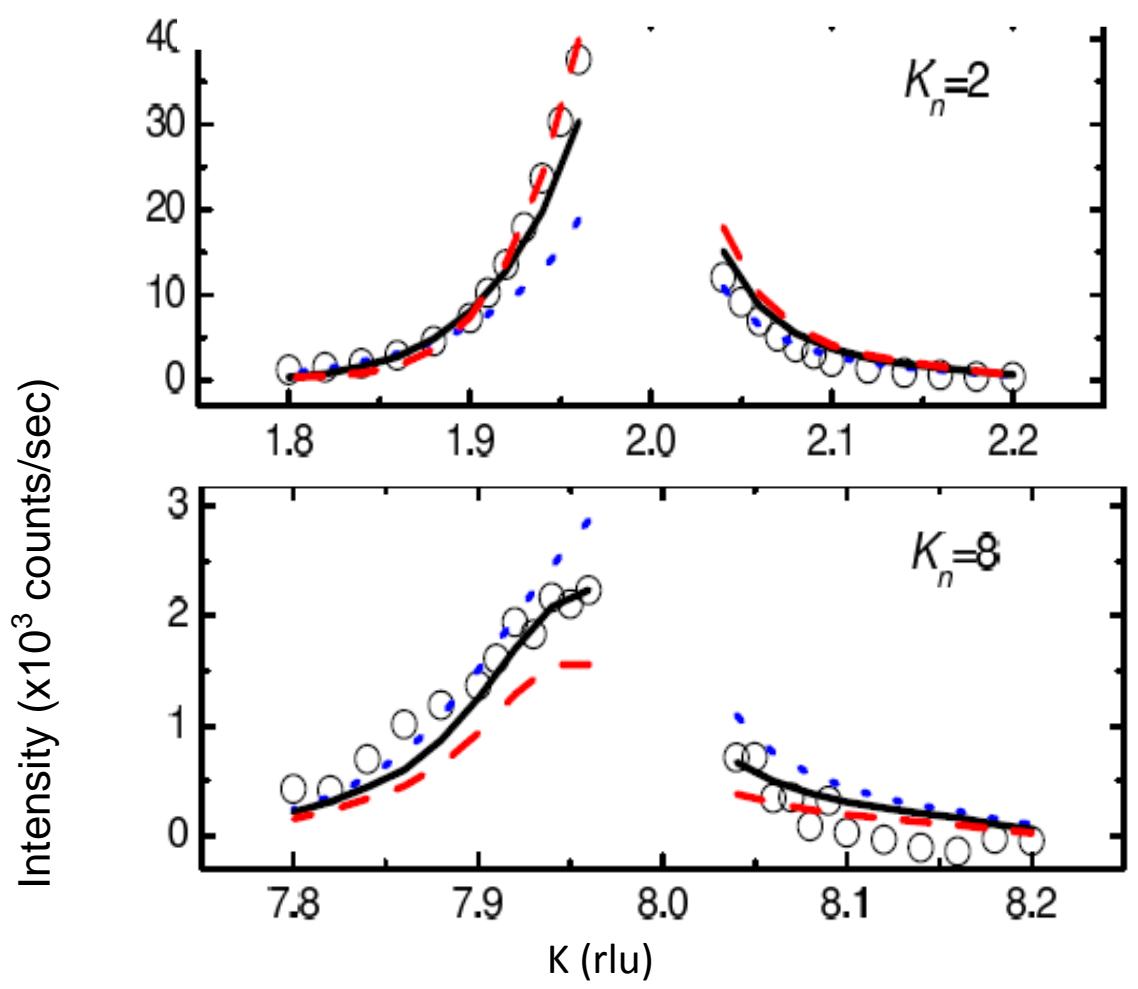

Figure 3.4: $\mathrm{X}$-ray diffuse $\mathrm{Ag} / \mathrm{Ag}(001)$ data (open circles) is fitted to a vacancy model over a large range of $\mathrm{Q}$. Black solid line shows excellent agreement with the data and yielded a vacancy cluster size of $750 \AA^{-3}$ and a very low vacancy concentration of 0.05\%. From Ref. [4].

vacancies, it would be useful to have deposition angle dependent for a series of coverages for this system. Also, for comparison sake, it would also be useful to have diffuse measurements for one of the other systems that showed multilayer structure growth.

Not sure what to make of it, but the Amar simulations and the X-ray diffuse both yield a very low vacancy concentration of $0.05 \%$. Simulations did not see such large vacancy cluster, probably, either due to the limited sample size that could be simulated, or there is a missing growth mechanism not yet established to explain the 
large vacancies.

\subsection{Missing material below exposed surface: Glanc- ing Angle Deposition technique}

Ideas, later described in Section 4.3, related to additional missing material below the exposed surface were inspired by GLAD, Glancing Angle Deposition technique[10]. GLAD is a fabrication technique in which growth is performed at oblique deposition angles $\left(\Theta_{D}>80^{\circ}\right)$ while the sample is rotating. It is primarily focused on creating three-dimensional mesoscopic columnar structures of the order of microns in height. Uniform microstructures can be created by taking advantage of shadowing which prevents deposited material from going into valleys. Examples of interesting shapes include helical columns, to screw columns or even zig-zag columns[?].

Additional missing material was shown to exist at the Si substrate for $100 \mathrm{~nm}$ $\mathrm{Si} / \mathrm{Si}$ (amorphous) deposited at glancing angles[16]. Scanning electron microscopy (SEM) and X-ray reflectivity characterization showed the film to be columnar, having a larger porosity (more missing material) near the substrate. It was as if the columnar structures were sitting on pedestals attached to the substrate. The columnar structured film naturally has a lower porosity than a bulk film (material is missing in between the columns), but there is also a noticeable additional missing material near the interface. It looks like the column is sitting on top of a smaller pedestal. This additional missing material at the base of the film will be exploited later.

GLAD is a novel and useful fabrication technique which is a possible example showing the interplay between diffusion and shadowing. However, GLAD produces 
amorphous structures and is not focused on the mechanisms controlling the first few layers of growth. To determine and characterize the importance of vacancies contributions to film strain, the focus will remain on crystalline metal film growth. Additional missing material at the buried interface may open up new possibilities for describing the buried interface.

\subsection{Conclusion}

The evidence so far appears to be inconclusive:

- X-ray reflectivity clearly showed a $1 \%$ compressive strain for three different noble metal surface morphology growth modes, multilayer and layer-by-layer, therefore it was inferred that vacancies were responsible for the observed strain.

- For the $\mathrm{Cu}(001)$ system the molecular dynamics simulation interpretation was that surface morphology was responsible for the $1 \%$ strain.

- X-ray diffuse scattering measurements confirmed the existence of very large vacancy clusters in relatively small concentrations that yielded a $1 \%$ strain on the $\mathrm{Ag} / \mathrm{Ag}(001)$.

- GLAD is a fabrication technique, but, showed that there is additional missing material at the substrate/film interface.

It seems that there may be an interplay between the surface morphology strain and the vacancy induced strain. A layer-by-layer growth system has not been studied, which could help to flush out and understand the strain contributions from the 
surface morphology and the vacancies as a function of deposition angle. With increasing deposition angle, the layer-by-layer system should start to have a larger surface morphology due to increased surface roughness, due to shadowing.

Questions that have not yet been addressed:

- Are vacancies responsible for the observed strain in systems where surface morphological structures (mounding) is suppressed?

- At what deposition angle does the surface morphology strain become important and can the strain contribution from buried vacancies be determined?

Research presented in the next chapter is the starting attempts at addressing these questions.

There is simply not enough conclusive information to say the film strain is solely due to vacancies. In order to determine if vacancies do play a role in the observed strain, a series of thorough experiments need to be performed as a function of varying deposition angle, from normal incidence to very large (glancing angle) for a series of film thickness. Also, a system that exhibits layer-by-layer growth $(\mathrm{Ag} / \mathrm{Ag}(001))$ would be the best choice since it will not be dominated by surface morphological structures at normal incidence deposition angles. 


\section{Chapter 4}

\section{Deposition Angle-Dependent Metal Film Growth}

\subsection{Overview}

Improving the current understanding of thin film growth by studying and modeling basic systems that show interesting growth phenomena is the focus of my research. Of particular interest is missing material buried below the film's exposed surface[4, 2] which can come in the form of vacancies, vacancy clusters, overhangs, or voids that are possibly connected to the surface. There is a wealth of research in the area of thin film growth and yet the lack of evidence for missing material below the exposed surface has prevented a proper understanding of the growth mechanisms leading to the incorporation of vacancies during thin film growth. Theoretical growth models intentionally remove voids, overhangs, or vacancies through mechanisms such as downward funneling[5]. This missing material can affect the physical properties of 
the film such as conductivity[1] and film strain[2]. I will show there is a significant amount of missing material below the exposed surface that has to be considered to properly understand thin film growth mechanisms.

There are two opposing schools of thought when it comes to understanding how film strain is incorporated during film growth: 1) structural surface morphology[3] and 2) vacancy clusters[4]. Amar's group has performed deposition angle dependent molecular dynamics simulations for thin film $\mathrm{Cu} / \mathrm{Cu}(100)[3]$ which attributes thin-film reflectivity oscillations (strain fields) to nano-surface structures. In these simulations, the structures deposited at glancing angles with respect to the surface look like ripples or hills and valleys perpendicular to the deposition direction. The length scale of these ripples for the simulated $60^{\circ}$ deposition angle is of the order of $3 \mathrm{~nm}$. The films used in these simulations are less dense with increasing deposition angle, and do not show missing material below the exposed surface. In contrast, X-ray diffuse measurements[4] on $\mathrm{Ag} / \mathrm{Ag} 100 \mathrm{ML}$ have shown that vacancy clusters buried below the exposed surface give rise to the strain seen in the film.

Is it surface morphology or is it vacancies that are responsible for the measured film strain? This is a complicated question with no direct answer. In order for this question to be addressed, the buried missing material has to be characterized.

Previous scanning electron microscope work done using GLAD [17, 15] showed deposition angle dependent columnar structures that grow very tall, of the order of microns, and have low densities, a lot of space in between the columns, for several hetero-epitaxial systems, using fast deposition rates at high temperatures. In contrast, my work focuses on what is occurring at the buried interface for thin films, of the order of ML at low deposition flux rates ( $20 \mathrm{ML} / \mathrm{min}$ ) for a variety of deposition angle 
dependence.

The key to the missing material is buried below the exposed surface and we want to expose it. We are interested in detecting and characterizing missing material at the buried interface. Additional experimental evidence for the existence of missing material below the exposed surface for varying deposition angles has also been shown. Deposition angle dependent X-ray reflectivity is able to measure a region that is sensitive to this missing material. The characterization of the $\mathrm{Ag} / \mathrm{Si}(111) 7 \times 7$ system using X-ray reflectivity along with AFM confirms missing material. The characterization of missing material in the $\mathrm{Ag} / \mathrm{Ag}(001)$ using $\mathrm{X}$-ray reflectivity as the primary investigation tool, is still in progress.

Our previous reflectivity and diffuse work $[2,4]$, all done at a fixed deposition angle, along with the deposition angle dependent MD simulations[3] were the motivation to experimentally characterize the deposition angle dependence of the surface morphology and vacancy strain contributions. These deposition angle dependent and coverage dependent reflectivity experiments are presented in Chapter 4.

\section{2 $\mathrm{X}$-ray reflectivity}

The reflection of X-rays from surfaces to characterize structural properties is accomplished by measuring the momentum transfer $\left(Q_{z}=k_{f}-k_{i}\right)$ perpendicular to the surface as shown in Figure 4.1. X-rays penetrate into thin films which provide information about the substrate, interface, and film properties such as electron density as a function of $z$ (perpendicular to the sample), film height, surface and interface roughnesses. As shown in Figure 4.2, total external reflection occurs below a critical 
momentum transfer $\left(Q_{c}\right)$ since the index of refraction is less than one for $\mathrm{X}$-rays. This critical momentum transfer squared is proportional to the electronic density, where

$$
Q_{c}^{2}=16 \pi \rho_{b}
$$

and $\rho_{b}$ is the scattering length density (SLD) $=\rho_{N} b, \rho_{N}$ is the atomic number density, and $b \approx r_{e} Z$ and $r_{e}$ is the classical electron radius. Also, the film height is inversely proportional to thin film fringe oscillation spacing ( film thickness $=\frac{2 \pi}{\Delta Q_{z}}$ ); and an increase in film roughness can be detected by looking at the dampening of the thin film oscillations and also a decrease in intensity at higher $Q_{z}$.

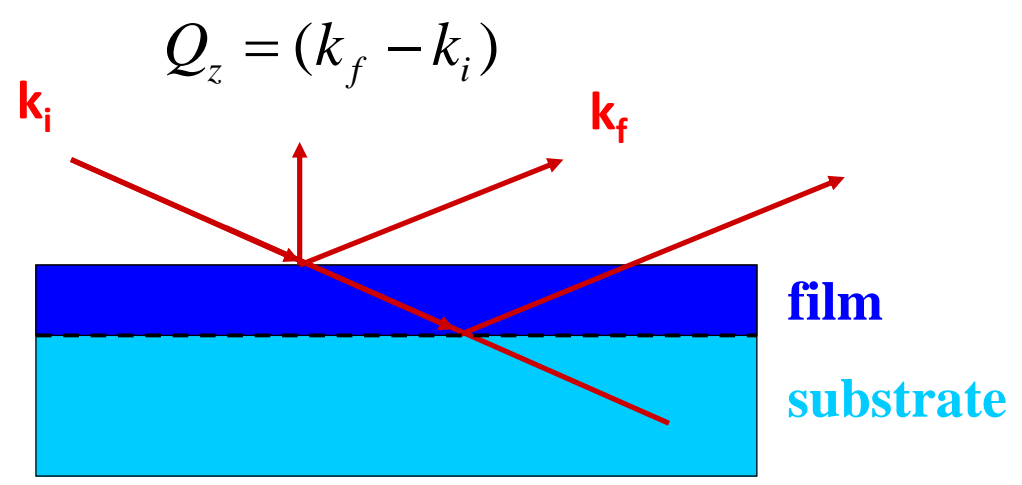

Figure 4.1: X-ray reflectivity measures the atomic scattering length density as a function of $z$, perpendicular to the surface and is independent of the in-plane structure. 


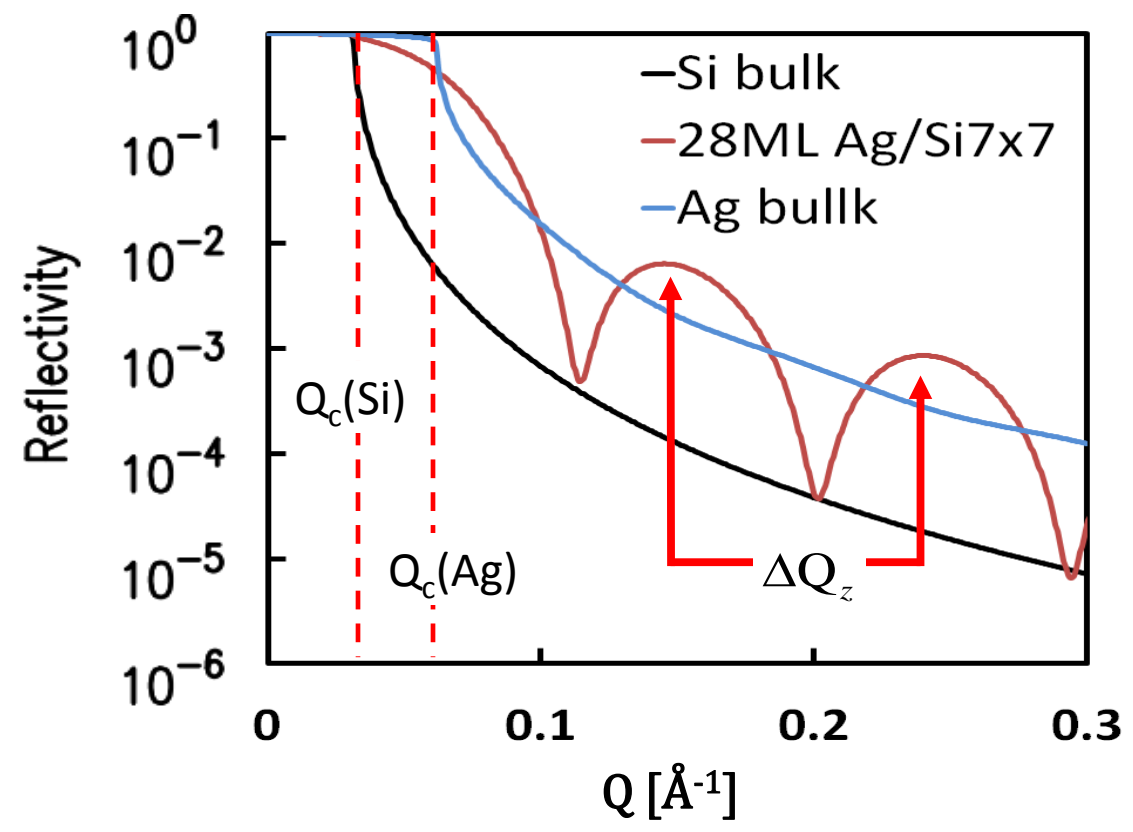

Figure 4.2: $\mathrm{X}$-ray reflectivity critical momentum transfer squared, $Q_{c}^{2}$ is proportional to the electron density $(\mathrm{Z})$. Higher Z, larger critical angle. Also shown are the thin film fringe spacing, $\Delta Q_{z}$, which is inversely proportional to the film thickness.

\subsection{Lower interfacial density of $\mathrm{Ag} / \mathrm{Si}(111) 7 \times 7$}

The $\mathrm{Ag} / \mathrm{Si}(111) 7 \times 7$ system was chosen to study due to the high electron density contrast which should make it easy to model the buried $\mathrm{Ag} / \mathrm{Si}$ interface using X-ray reflectivity. Several deposition angle dependent RT samples were prepared in order to help characterize the missing material below the exposed surface.

\subsubsection{Ag/Si experimental details}

Samples 30A and 80A were prepared in UHV by first creating the $7 \mathrm{x} 7$ reconstructed Si surface by flash annealing to $1200^{\circ} \mathrm{C}$, and then depositing the $\mathrm{Ag}$ film at room 
temperature for two different deposition angles, $\Theta_{D}=30^{\circ}$ and $80^{\circ}$ (an uncertainty of $\pm 5^{\circ}$, due to the way the evaporator is mounted) with respect to the sample normal. During the deposition, the thermal evaporator (resistively heated crucible) was kept at constant temperature $\left(1060^{\circ} \mathrm{C}\right)$. The evaporator calibration was done at $\Theta_{D}=30^{\circ}$ using a calibrated quartz monitor and also corroborated by measuring hetero-epitaxial thin film oscillations which yielded an evaporation rate of $22 \mathrm{ML} / \mathrm{min}$. The $\Theta_{D}=$ $80^{\circ}$ deposition flux rate was calculated from a $\cos \left(\Theta_{D}\right)$ projection correction of the $\Theta_{D}=30^{\circ}$ calibrated rate. The targeted coverage of sample $30 \mathrm{~A}$ was $28 \pm 3 \mathrm{ML}$, where uncertainty is based on the calibration. For sample 80A an additional correction based on the uncertainty of the deposition angle yielded a targeted coverage of $28 \pm 14 \mathrm{ML}$. The large coverage error for glancing angle deposition is due to the evaporation rate projection correction: $\cos \left(85^{\circ}\right)$ vs $\cos \left(80^{\circ}\right)$ yields a $50 \%$ coverage error.

After sample preparation in UHV, low-angle X-ray reflectivity and atomic force microscopy (AFM) measurements were performed in air. Low-angle X-ray reflectivity data was measured on a four circle diffractometer using a $18 \mathrm{~kW}$ Molybdenum $\mathrm{K}_{\alpha 1}$ rotating anode line-focus beam with a Ge(111) monochromator using a Bicron detector. The Asylum Research Cypher AFM was operated in tapping mode utilizing an Olympus OMCL-AC240TS-C2 AFM cantilever tip, which has a nominal tip radius of $6.8 \mathrm{~nm}$ and angle of $30^{\circ}$. Also, another sample $30 \mathrm{U}(21 \pm 2 \mathrm{ML} \mathrm{Ag} / \mathrm{Si} 7 \mathrm{x} 7$ deposited at $\Theta_{D}=30^{\circ}$ ) was similarly prepared and then extended-range $\mathrm{X}$-ray reflectivity was measured in situ using the surface scattering chamber (base pressure of $1 \times 10^{-10}$ Torr) on a Psi diffractometer located at the 6-IDC beam line at the Advanced Photon Source. The photon energy was $16.2 \mathrm{keV}$ and the data was collected using a Bicron detector. 


\subsection{2 $\mathrm{X}$-ray reflectivity results: Overview}

The reflection of X-rays from surfaces to characterize structural properties is accomplished by measuring the momentum transfer $\left(Q_{z}=k_{f}-k_{i}\right)$ perpendicular to the surface. X-rays penetrate into thin films, providing information about the substrate, interface, and film properties, such as: electron density as a function of $z$ (perpendicular to the sample), film height, surface and interface roughnesses. Total external reflection occurs below a critical momentum transfer $\left(Q_{c}\right)$ since the index of refraction is less than one for X-rays. This critical momentum transfer squared is proportional to the electronic density, where $Q_{c}^{2}=16 \pi \rho_{b}, \rho_{b}$ is the scattering length density $=\rho_{N} b$, $\rho_{N}=$ atomic number density, and $b \approx r_{e} Z$ and $r_{e}$ is the classical electron radius. Also, the film height is inversely proportional to thin film fringe oscillation spacing (film thickness $\left.=\frac{2 \pi}{\Delta Q_{z}}\right)$; and an increase in film roughness can be detected by looking at the dampening of the thin film oscillations and also a decrease in intensity at higher $Q_{z}$.

As a function of increasing deposition angle, the X-ray reflectivity data, shown in Figure 4.3, implies that the Ag films are less dense, thicker, and rougher, indicating a change in the surface morphology. The thin film fringe spacing is becoming shorter as the deposition angle increases corresponding to thicker films. The critical momentum transfer decreases with the increasing deposition angle, suggesting the average $\mathrm{Ag}$ film density decreases concomitantly with the increasing thicker films. The critical momentum transfer for bulk $\mathrm{Ag}$ is $\sim 0.06 \AA^{-1}$ which occurs at the point where total external reflection stops and is sharply seen for Ag films over 50ML due to penetration depth. This is hard to see in the Figure, but by looking closely at the critical momentum transfer of bulk $\mathrm{Si}$, near $Q_{z}=0.03 \AA^{-1}$, one can see that sample 80A 


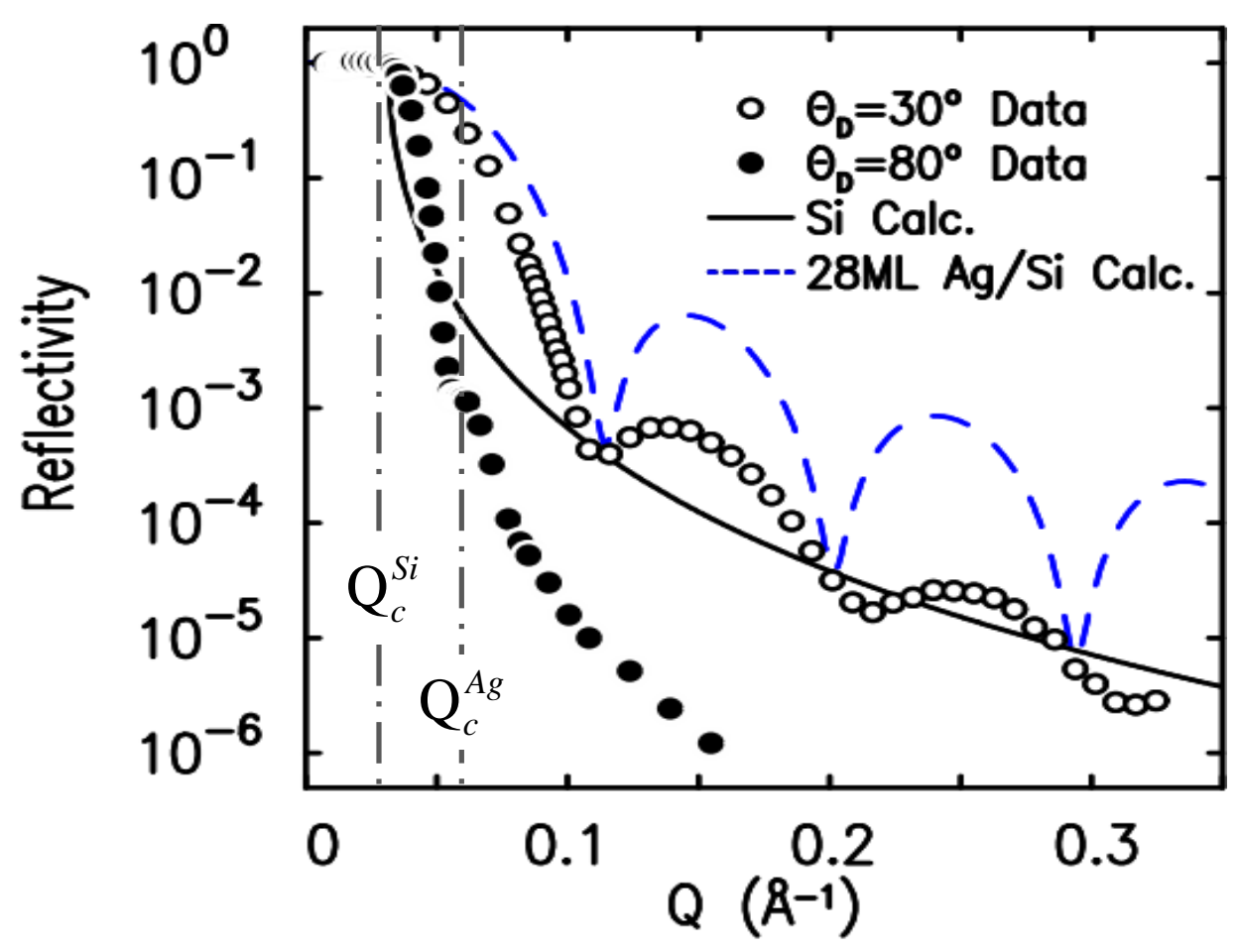

Figure 4.3: Low angle $\mathrm{X}$-ray reflectivity measured on two $28 \mathrm{ML} \mathrm{Ag} / \mathrm{Si}(111) 7 \mathrm{x} 7$ samples deposited at different deposition angles w.r.t. the sample normal, grazing incidence $\Theta_{D}=80^{\circ}$ (solid circles) and $\Theta_{D}=30^{\circ}$ (open circles). Also shown for comparison are reflectivity calculations for bulk $\mathrm{Si}$ (solid black line) and 28ML Ag on Si7x7 (blue dashed line). For the $80^{\circ}$ deposition, the fall-off from total reflection occurs much sooner suggesting a lower density of $\mathrm{Ag}$ than for the $30^{\circ}$ deposition angle. 
critical momentum transfer is slightly higher than bulk Si and sample 30A critical momentum transfer is larger than $80 \mathrm{~A}$, but less than the calculated $28 \mathrm{ML}$ bulk $\mathrm{Ag}$ critical momentum transfer. Also inferred, is a substantial increase in film roughness as a function of increasing deposition angle since the $\Theta_{D}=30^{\circ}$ thin film oscillation intensity is smaller than the perfect $28 \mathrm{ML} \mathrm{Ag} \mathrm{film,} \mathrm{while} \mathrm{the} 80 \mathrm{~A}$ thin film oscillation intensity is barely visible.

\subsubsection{Scattering Length Density Model}

A quantitative analysis of the reflectivity data will be presented in order to extract nanoscale information about the sample, such as film thicknesses, depth dependent density and inter-facial roughness. After modeling the scattering length density profile $\left(\rho_{b}(z)\right)$, the low angle specular reflectivity was calculated by the Parratt method[18] using a software package Reflpak $[19,20]$ by finely dividing $\rho_{b}(z)$ into a series of thin dielectric slabs.

Using the above observations, a general scattering length density profile given by

$$
\rho_{b}(z)=\rho_{b}^{S i}(z)+\rho_{b}^{f i l m}(z)
$$

was constructed in order to model the salient features of the low angle $\mathrm{X}$-ray reflectivity deposition angle dependent $\mathrm{Ag} / \mathrm{Si}(111) 7 \times 7$ data, as shown in Figure 4.5. At low angles, the details of the Si7x7 layer can be neglected since the atomic scale is much shorter than the probing length scale of the present experiment. X-ray scattering studies[21] have shown that the $\mathrm{Ag} / \mathrm{Si}$ interface is atomically abrupt, therefore, it was assumed that the first Ag atomic layer starts immediately at $z>0$. A large sur- 
face roughness was required to dampen the thin film oscillations which also dropped the intensity at the high $Q_{z}$ tail. However, the data showed a much lower intensity than calculated. To reproduce the lower intensity at the high $Q_{z}$ tail, there must be a broader transition at the $\mathrm{Ag} / \mathrm{Si}$ interface. Therefore, a $\mathrm{Ag}$ transitional layer at the $\mathrm{Ag} / \mathrm{Si}$ interface was introduced. There are essentially two regions of $\mathrm{Ag}$ on $\mathrm{Si}$ : a transitional layer $D_{0}$, which as an abrupt interface with the $\mathrm{Si}$ and the majority of the film layer, $D_{1}$. As will be shown later, $\rho_{b}(z)$ from X-ray reflectivity matches the AFM profile for the exposed surface. Of course, information of the buried transitional layer at the $\mathrm{Ag} / \mathrm{Si}$ interface is not accessible to the AFM.

Putting these features together, we now have the $\rho_{b}(z)$ model that was used to analyze the data as shown in Figure 4.5, consists of three layers: 1) a layer of semiinfinite $\mathrm{Si}$, and two Ag film layers: 2) a transitional layer, followed by 3) the top Ag film layer. Each layer is defined by a scattering length density $\rho_{b}^{i}$, a thickness $D_{i}$, an rms roughness $\sigma_{i}$, and a linear absorption $\mu_{i}$, where $i$ denotes the layer index. The $\mathrm{Ag}$ film is described as follows:

$$
\rho_{b}^{f i l m}(z)= \begin{cases}\frac{\rho_{b}^{1}-\rho_{b}^{0}}{2}\left[\operatorname{erf}\left(\frac{z-z_{0}}{\sqrt{2} \sigma_{0}}\right)+1\right]+\rho_{b}^{0}, & \text { for } 0<z<z^{\prime} \\ \frac{\rho_{b}^{1}}{2}\left[\operatorname{erf}\left(\frac{z_{1}-z}{\sqrt{2} \sigma_{1}}\right)+1\right], & \text { for } z \geq z^{\prime} .\end{cases}
$$

where, erf is the error function, $z_{i}$ is the center of $i$ th error function, and $z^{\prime}$ is where the two error functions converge to the same value.

An alternative (and common) explanation for the lower Ag transitional layer at the $\mathrm{Ag} / \mathrm{Si}$ interface is that there is mixing of these layers. This does not occur on Si7x7 because the $7 \times 7$ layers remains intact after $\mathrm{Ag}$ deposition [21].

Key features of the X-ray reflectivity data are particularly sensitive to some of the 


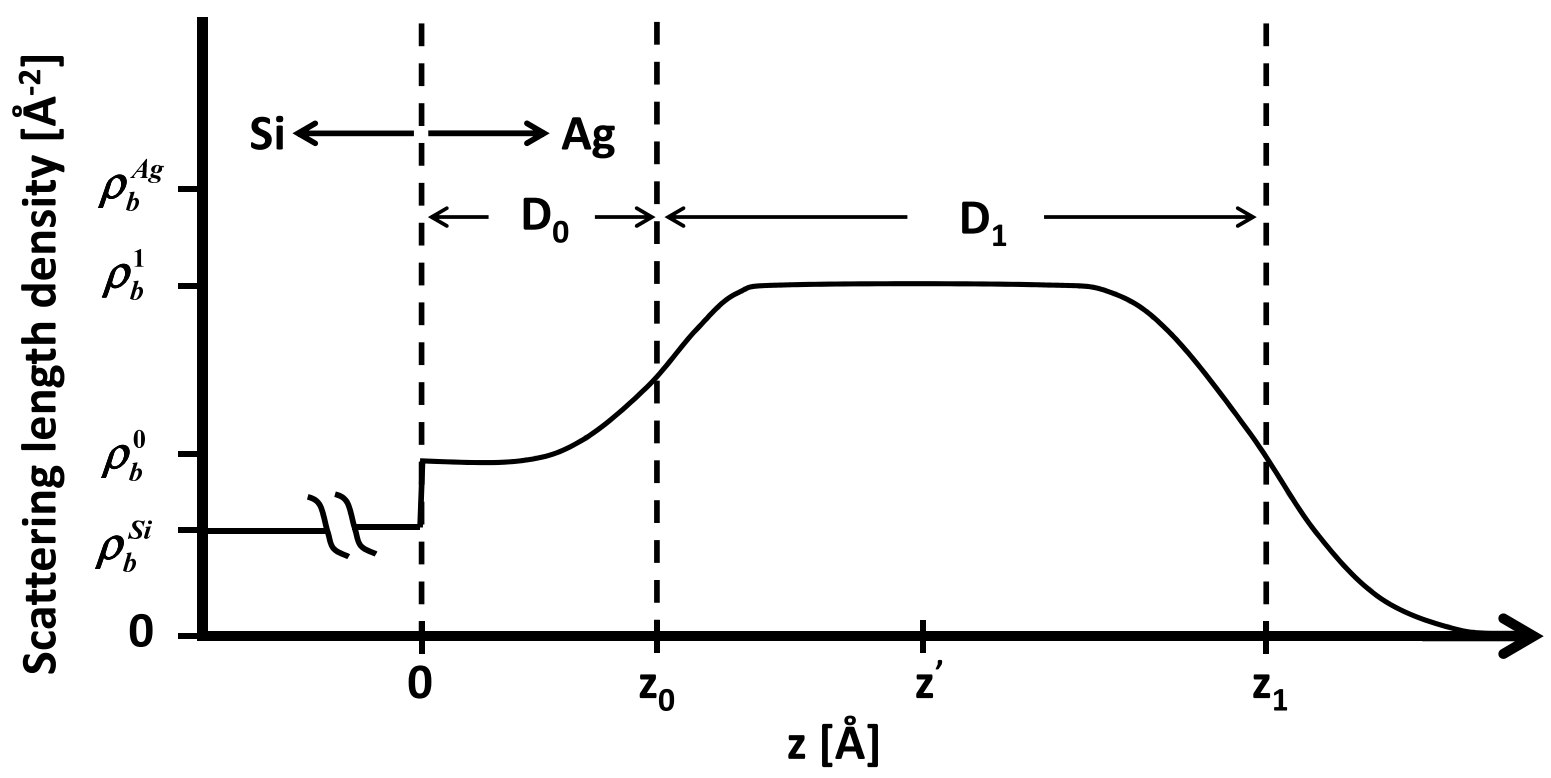

Figure 4.4: Figure is the general outline of the $\mathrm{Ag} / \mathrm{Si}(111) 7 \times 7$ SLD profile model used in reflectivity calculations.

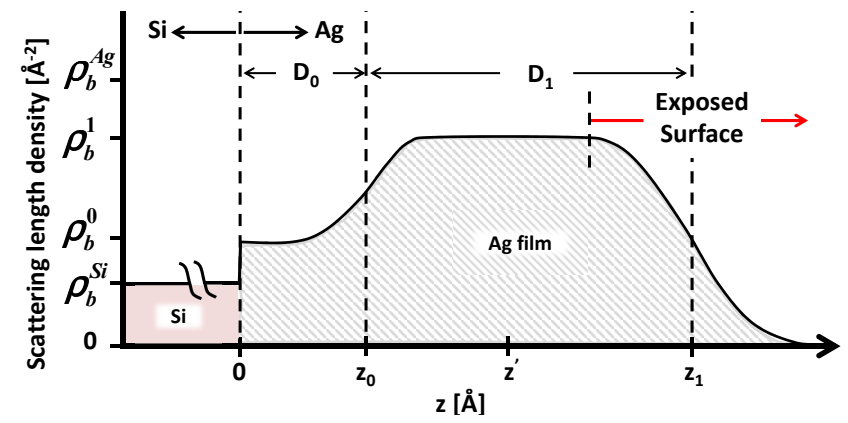

Figure 4.5: The scattering length density profile, $\rho_{b}(z)$, used to model the X-ray reflectivity shows a lower density at the $\mathrm{Ag} / \mathrm{Si}$ interface. The lower density below the exposed surface $\left(z<z^{\prime}\right)$ is probably due to missing material that forms immediately during deposition. The $\rho_{b}(z)$ does not distinguish the type of missing material: voids, vacancies, vacancy clusters, etc. $\mathrm{D}_{0}$ is the lower density $\mathrm{Ag}$ transitional layer and $\mathrm{D}_{1}$ makes up the bulk of the Ag film. 
$\rho_{b}(z)$ model parameters, which allowed a good first guess at these parameters before least squares fit refining. $D_{1}$ was initially chosen from the observed fringe spacing and the critical angle is sensitive to $\rho_{b}^{1}$, since both of these parameters are responsible for the majority of density of the Ag film. Increasing the surface roughness $\sigma_{1}$ reduced the height of thin film oscillations to match the data. Another striking feature was the large decay of the reflectivity data at high $Q_{z}$ which was not captured by the top-layer surface roughness $\sigma_{1}$. The high $Q_{z}$ intensity could only be decreased by including the lower $\mathrm{Ag}$ scattering length density at the $\mathrm{Ag} / \mathrm{Si}$ interface: $\rho_{b}^{0}<\rho_{b}^{1}$.

Several interesting observations can be made by taking a closer look at the general $\rho_{b}(z)$. The film surface is exposed to surface-only probing tools for $z>z^{\prime}$. The total coverage is calculated by integrating $\rho_{b}^{f i l m}(z), \Theta=\frac{1}{\rho_{b}^{A g}} \int \rho_{b}^{f i l m}(z) d z$.

\subsection{4 $\Theta_{D}=30^{\circ}$}

After refinement of the $\rho_{b}(z)$ model, a good fit to sample 30A X-ray reflectivity data was obtained, as shown in Figure 4.6. Excluding the lower Ag density transitional layer, shown by the dot-dashed line, the model provided a poor fit to the data at high $Q_{z}$. A better fit to the high $Q_{z}$ data could be obtained by adding the lower $\mathrm{Ag}$ transitional inter-facial layer and allowing $\rho_{b}^{0}$ to start at the same value of the bulk Si $\rho_{b}^{S i}$ ) at $z=0$, shown by the blue dashed line. However, the best fit was obtained by putting in an abrupt $\mathrm{Ag}$ transition $\left(\rho_{b}^{0}>\rho_{b}^{S i}\right)$ at $z=0$, as shown by solid black line. Model parameters for the best fit are recorded in 4.1. 


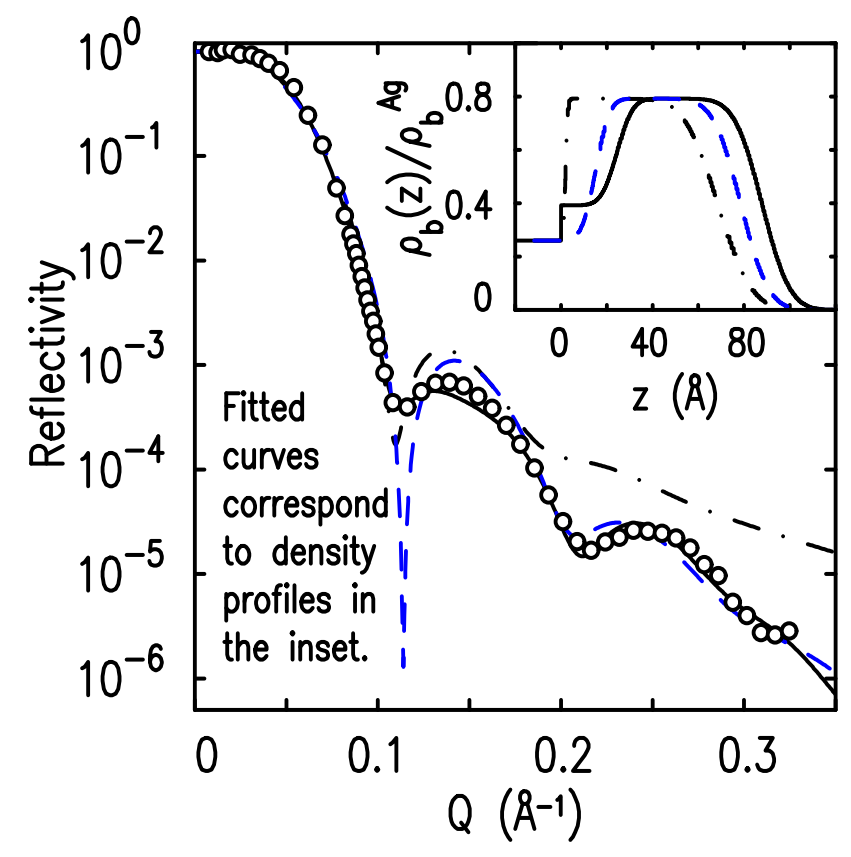

Figure 4.6: Sample 30A X-ray reflectivity data shown in open circles and the 3 lines are reflectivity calculations using the respective $\rho_{b}(z)$ in the inset. The black dotdashed fit corresponds to a uniform Ag film, where $\rho_{b}^{\text {film }}$ starts at the average film density, $\rho_{b}^{1}$, at the Si interface, which is not a good fit since it misses the data at $Q_{z}=0.3$. The blue-dashed line has the lower Ag interfacial layer added and starts at the bulk Si density which improves the fit, but over shoots the cusp at $Q_{z}=0.1$. The best fit if the solid black line which has the lower interfacial layer which has $\rho_{b}^{0}>\rho_{b}^{S i}$, but $\rho_{b}^{0}<\rho_{b}^{1}$. This abrupt transitions reduces the depth of the cusp at $Q_{z}=0.1$.

Surprisingly, the Ag transitional layer was 13ML thick: $\rho_{b}^{0}$ was $40 \%$ of the bulk Ag density at the $\mathrm{Ag} / \mathrm{Si}$ interface for the first 5ML of $\mathrm{Ag}$, followed by a transition to $80 \%$ of bulk Ag for $\rho_{b}^{1}$, which corresponds to $\sim 4 \mathrm{ML}$ of missing material below the exposed surface, according to Equation 4.6. Along with the decreased density, the total layer thickness was $30 \%$ taller than an ideal 28ML bulk Ag film, and the surface roughness was 4ML. Integrating $\rho_{b}^{f i l m}(z)$ yields a total coverage of $28 \mathrm{ML}$, in agreement with the calibration even though the coverage was not constrained.

$\mathrm{Ag}$ is fairly inert in atmosphere; however, in order to address potential contam- 
Table 4.1: Table shows sample 30A, 30U, and 80A X-ray reflectivity best fit $\rho_{b}(z)$ model parameters. The table quantitatively shows with increasing deposition angle a decrease in the film density, increase in the surface and interfacial roughness, and an increase in the apparent film thickness. Important to note is that all three samples showed missing material below the exposed surface which was required to obtain good fits to the $\mathrm{X}$-ray reflectivity data. Uncertainties are shown in parenthesis.

\begin{tabular}{l|l|c|l|l|c|l|l|l|l|l}
\hline $\begin{array}{l}\text { Sample } \\
\text { Name }\end{array}$ & $\begin{array}{l}\Theta_{D} \\
{\left[{ }^{\circ}\right]}\end{array}$ & $\rho_{b}^{0} / \rho_{b}^{A g}$ & $\begin{array}{l}\mathrm{z}_{0} \\
{[\mathrm{ML}]}\end{array}$ & $\begin{array}{l}\sigma_{0} \\
{[\mathrm{ML}]}\end{array}$ & $\rho_{b}^{1} / \rho_{b}^{A g}$ & $\begin{array}{l}\mathrm{z}_{1} \\
{[\mathrm{ML}]}\end{array}$ & $\begin{array}{l}\sigma_{1} \\
{[\mathrm{ML}]}\end{array}$ & $\begin{array}{l}\mathrm{z}_{\max } \\
{[\mathrm{ML}]}\end{array}$ & $\begin{array}{l}\Theta \\
{[\mathrm{ML}]}\end{array}$ & $\begin{array}{l}\Theta_{M M} \\
{[\mathrm{ML}]}\end{array}$ \\
\hline $30 \mathrm{U}$ & $30(5)$ & 0.65 & 9 & 1.7 & 0.8 & 30.5 & 2.5 & 38 & 23 & 1 \\
\hline $30 \mathrm{~A}$ & $30(5)$ & 0.4 & 10 & 2.3 & 0.8 & 37 & 4 & 49 & 25 & 4 \\
\hline $80 \mathrm{~A}$ & $80(5)$ & 0.3 & 19 & 7.4 & 0.4 & 94 & 20 & 155 & 38 & 3 \\
\hline
\end{tabular}

ination issues due to making measurements in air, extended-range reflectivity was measured in situ in UHV for sample 30U. It will be shown that it has a similar $\rho_{b}$ profile, therefore, we believe the in-air measured buried inter-facial missing material is not caused by in-air contamination.

The Si7x7 reconstructed surface will be included in the modeling since areas of the extended-range $\mathrm{X}$-ray specular reflectivity are sensitive to the $7 \mathrm{x} 7$ details. The extended-range specular reflectivity was calculated[22] from the square of the sum of three complex amplitudes: 1) the bulk semi-infinite Si substrate, 2) the Si7x7 discrete layers, and 3) the Ag film discrete layers, i.e.

$$
R\left(Q_{z}\right) \propto \frac{\left|V_{0}\right|^{2}}{Q_{z}^{2}}\left|f_{S i}\left(A_{S i}+A_{S i 7 x 7}\right)+\left(\frac{\rho_{S i}}{\rho_{A g}}\right)^{2} f_{A g} A_{A g}\right|^{2}
$$

where $f$ 's are the form factors that include the thermal Debye Waller factor $e^{-M}$, $\rho$ 's are the areal densities, and A's are the complex amplitudes. The substrate roughness $V_{0}$ was found to be negligible for the Si substrates and was set to 1 . The 
semi-infinite bulk Si amplitude is:

$$
A_{S i}=\frac{\left(1+e^{-i Q_{z} d_{S i} / 12}\right)\left(1+e^{-i Q_{z} d_{S i} / 3}\right)\left(1+e^{-i Q_{z} d_{S i} 2 / 3}\right)}{1+e^{-i Q_{z} d_{S i}}}
$$

and the Si7x7 surface reconstruction is:

$$
A_{S i 7 x 7}=\sum_{j} \eta_{j} e^{-i Q_{z} z_{j}} e^{-Q_{z}^{2} \zeta_{j}^{2} / 2}
$$

where $j$ is the index number for an atomic layer in the $z$-direction, perpendicular to the surface $(z=j d), d$ is the inter-atomic layer spacing. The Robinson [23] values were used for the $\operatorname{Si7x} 7$ parameters: $\left(\eta_{j}\right)$ is the Si density at layer $j$, the Si layer $j$ roughness is $\left(\zeta_{j}\right)$, and the $7 \times 7$ layer spacing is $\left(z_{j}\right)$. The Ag film amplitude is:

$$
A_{A g}=\sum_{j} \frac{\rho_{b}^{f i l m}\left(j d_{f i l m}\right)}{\rho_{b}^{A g}} e^{-i Q_{z} j d_{f i l m}}
$$

where $\rho_{b}^{f i l m}$ is the scattering length density profile characterized by Equation 4.1.

The Ag film $\rho_{b}(z)$ modeling started by simply rescaling sample $30 \mathrm{~A} \rho_{b}$ profile in the $z$-direction based on the coverage ratio, i.e. $\rho_{b}\left(\frac{28}{21} z\right)$. Then, the parameters were allowed to vary except for $\rho_{b}^{1}$. We did not have the critical angle data to nail down $\rho_{b}^{1}$, so it was fixed to the in air density of $0.8^{*} \rho_{b}^{A g}$. Regardless of the value of $\rho_{b}^{1}, \rho_{b}^{0}$ was always less than $\rho_{b}^{1}$.

After refining the Ag film parameters, a good fit to the extended-range reflectivity data was obtained as shown in Figure 4.7. The lower transitional interface obtains the correct Ag oscillation decay rate. Similar to sample 30A $\rho_{b}$ profile, excluding 
the lower Ag density transitional layer (dot-dashed black line) provided too high an intensity at the anti-Bragg locations $Q_{z}=1$ and 4 . Allowing $\rho_{b}^{0}$ to go to $\rho_{b}^{S i}$, blue dashed line, lowered the anti-Bragg intensity too much. The best fit required the abrupt transition at the $\mathrm{Ag} / \mathrm{Si}$ interface where, $\rho_{b}^{S i}<\rho_{b}^{0}<\rho_{b}^{1}$. Fit parameters for the best fit are recorded in Table 4.1.

The lower density at the $\mathrm{Ag} / \mathrm{Si}$ interface was absolutely needed to obtain a good fit to the UHV data. Contamination at the $\mathrm{Ag} / \mathrm{Si}$ buried interface for sample $30 \mathrm{~A}$ was ruled out since sample $30 \mathrm{U}$ measurements required the $\rho_{b}^{0}$ to be lower than the average $\rho_{b}^{1}$. With confidence, analysis of sample $80 \mathrm{~A}$ data proceeded in order to observe how the lower interface layer changed by increasing the deposition angle.

\subsection{5 $\Theta_{D}=80^{\circ}$}

Next, sample $80 \mathrm{~A}$ X-ray reflectivity measurements were analyzed using the same model and fitting technique as in Section 4.3.4. A good fit to the reflectivity data can be seen in Figure 4.8. All the prominent features of the X-ray data were sufficiently fit: the critical angle, the thin film fringe and the high $Q_{z}$ tail. The Ag film started above the Si density and then slowly transitioned over 10ML to a maximum film density of $44 \%$ bulk Ag. Due to such a low average Ag film density, the maximum film height was 150ML, 7 times larger than the expected coverage, with an rms roughness of 20ML. The 38ML coverage was within error bars of the calculated calibration. Again, the abrupt lower density at the $\mathrm{Ag} / \mathrm{Si}$ interface was required to fit the data. Fit parameters for the best fit are recorded in Table 4.1.

Within hours of X-ray measurements, sample 80A was also measured with atomic force microscopy to provide complementary and supporting evidence for the X-ray 

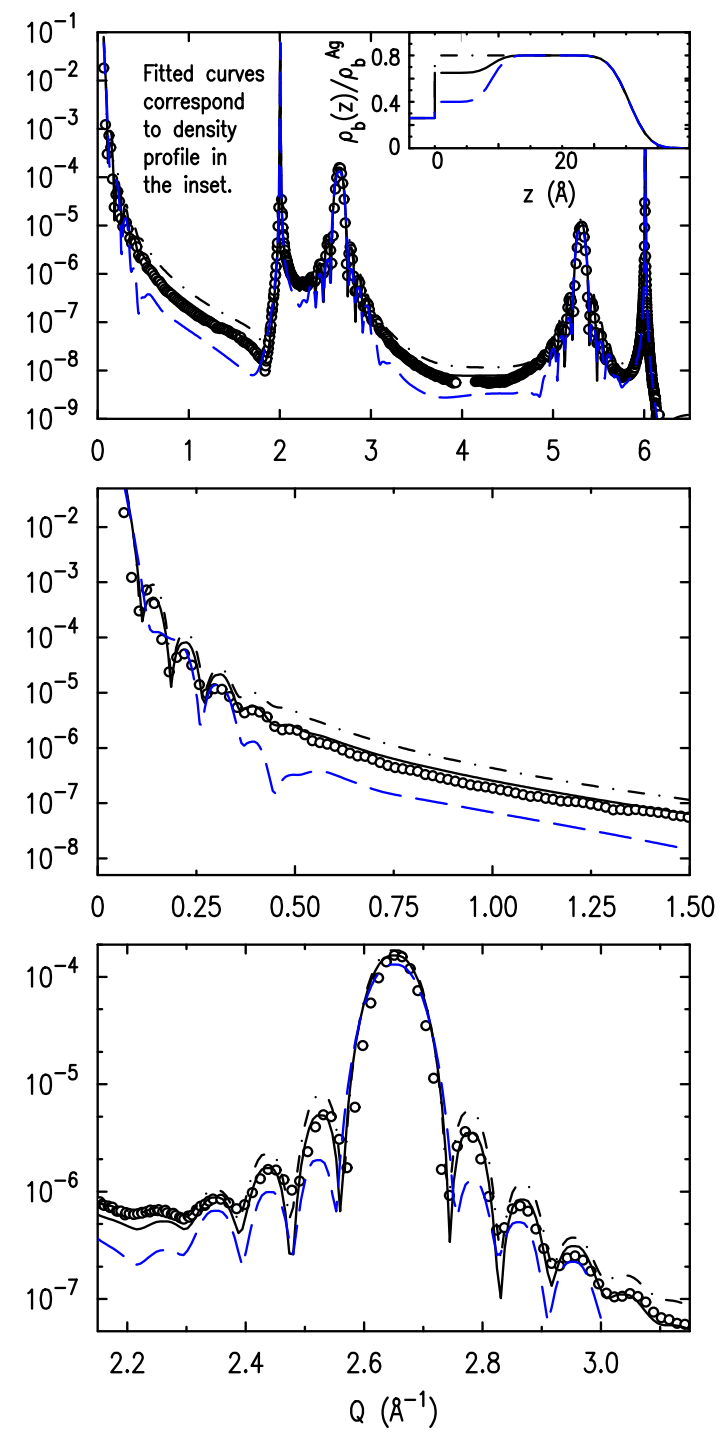

Figure 4.7: Upper panel shows sample 30U extended-range UHV X-ray reflectivity measurements (open circles) along with three calculated reflectivity curves using the upper panel inset respective $\rho_{b}$ profiles. The middle and bottom panels are zoomed in to show the details of the reflectivity curves. The two dashed $\rho_{b}$ profiles are poor fits to the data and miss the intensity near the regions $Q_{z}=1.0$ and 4.0, either too high (black dashed line) or too low (blue dashed line) while, the solid black line is the best fit. The solid line calculation is the only one to pick up the $\operatorname{Ag}(200)$ oscillation intensities. Also, the best fit yields $\sim 1 \mathrm{ML}$ of missing material below the exposed surface, which is small but essential to the fitting of the extended-range reflectivity. 
reflectivity results. The AFM measured rms surface roughness matched the X-ray reflectivity. The AFM results confirmed the $\mathrm{X}$-ray reflectivity apparent film height increase and large film roughness. In order to see what else could be learned about the low density lower interface, a quantitative comparison and analysis of the $\mathrm{X}$-ray and AFM data was performed. Surface height profiles for several images where averaged to provide the $\mathrm{P}_{j}$ profile shown in Figure 4.9(c).

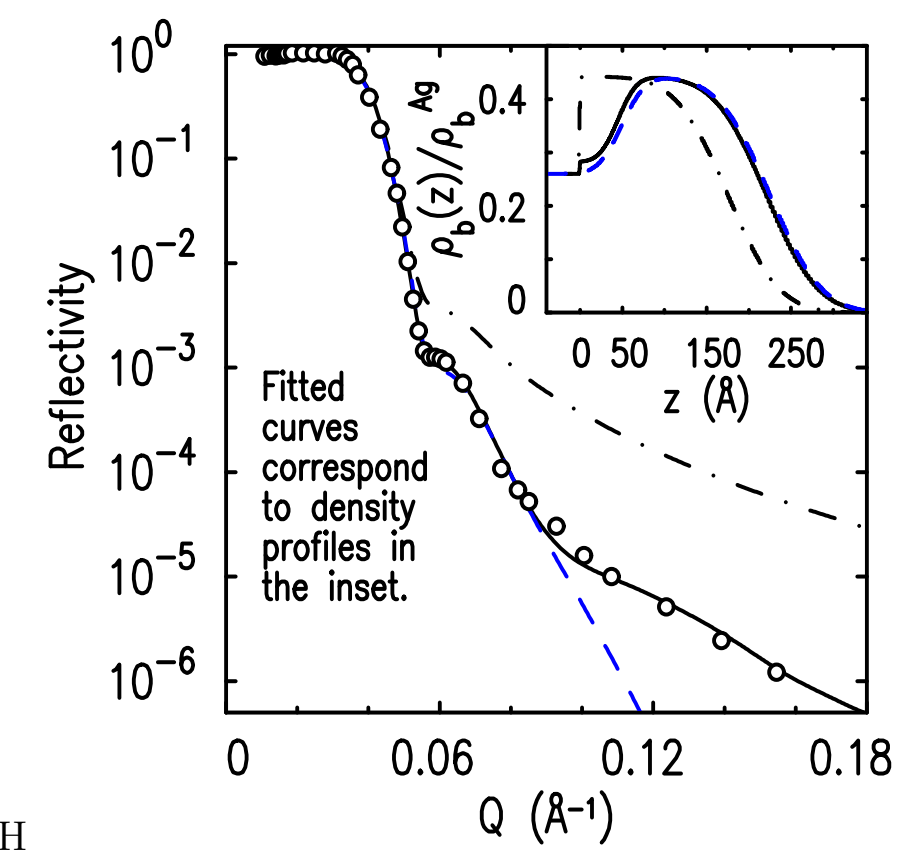

Figure 4.8: Sample 80A X-ray reflectivity data is shown in open circles and three calculated reflectivity using the $\rho_{b}(z)$ models in the inset. A nice fit (solid line) to the $\mathrm{X}$-ray data fits the critical angle well, along with the thin film fringe and the high $Q_{z}$ tail. The other density profiles miss the high $Q_{z}$ tail. 

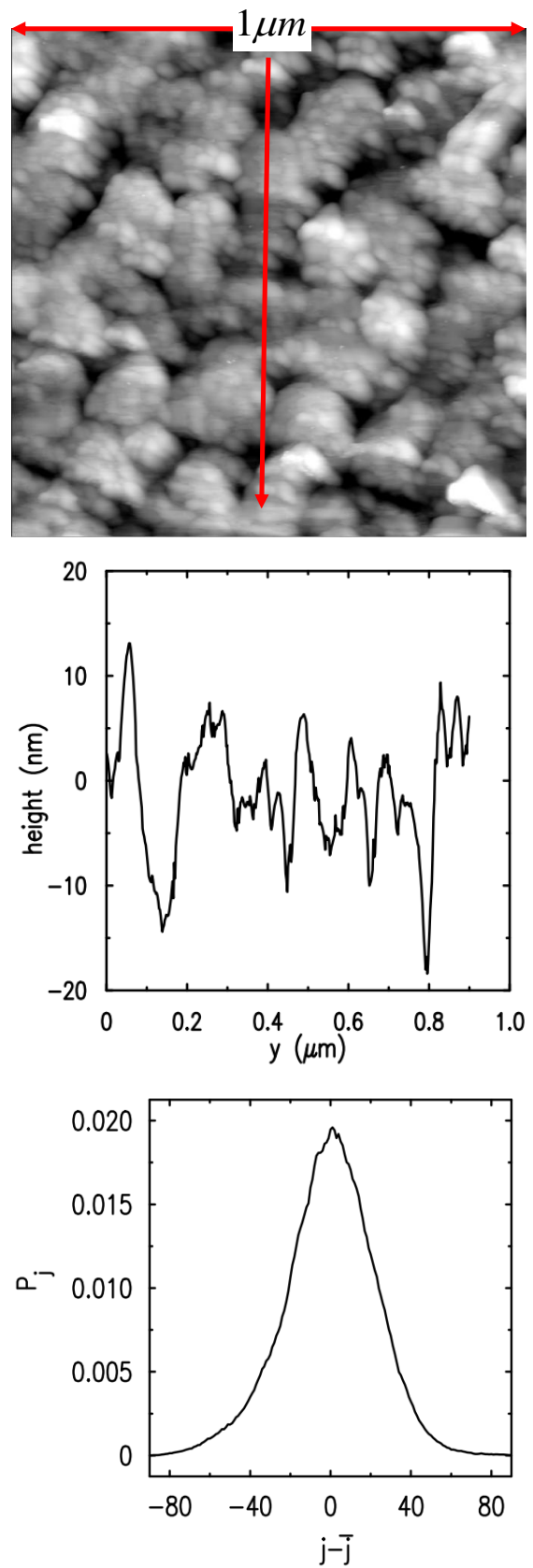

Figure 4.9: a) Upper panel shows an AFM image of sample 80A, b) middle panel shows a height profile trace of the red line on the AFM image (a), and c) shows the exposed surface height profile $\left(P_{j}\right.$ distribution). AFM image shows an average height of $66 \mathrm{ML}(15.6 \mathrm{~nm})$, rms roughness of $27 \mathrm{ML}(6.4 \mathrm{~nm})$ and a maximum height of $131 \mathrm{ML}$ (31nm) and supports the $\mathrm{X}$-ray reflectivity results. 


\subsubsection{Exposed surface fraction model: comparison of $\mathrm{X}$-ray reflectivity and AFM measurements}

Atomic force microscopy $(\mathrm{AFM})$ and $\mathrm{X}$-ray reflectivity measurements can be compared quantitatively through appropriate interpretation. AFM directly measures a height profile which is the exposed surface fraction distribution, $P_{j}$, where $j$ is the index number for an atomic layer in the $z$-direction. Whereas, the X-ray reflectivity, through modeling, yields the scattering length density profile, $\rho_{b}(z)$, which is then related to the atomic layer occupancy distribution, $\theta_{j}$, by normalizing the scattering length density in the $j$-th layer, $\rho_{b}(j)$, to the bulk scattering length density given by

$$
\theta_{j} \equiv \frac{\rho_{b}^{f i l m}(j)}{\rho_{b}^{A g}}
$$

The exposed surface fraction $P_{j}$ is obtained by taking the difference of two corresponding atomic layer occupancies $P_{j}=\theta_{j}-\theta_{j+1}$ as shown in Figure 4.10. This definition leads to several useful relationships. The total film coverage is equal to the sum of all individual atomic film layer occupancies, which can also be shown is equal

to sum of all $j * P_{j}$, i.e. $\Theta=\sum_{j=0}^{\infty} \theta_{j}=\sum_{j=0}^{\infty} j P_{j}$. Also, the sum of all exposed surface fractions is normalized to one:

$$
\sum_{j=0}^{\infty} P_{j} \equiv 1
$$

It should be noted that missing material below the exposed surface, such as overhangs or voids, would appear as a negative $P_{j}$. Unlike the AFM, which is probing the exposed surface and cannot see material below the surface, X-ray scattering penetrates the surface and can detect the density of buried layers. However, the modeled $\mathrm{X}$-ray reflectivity $\theta_{j}$ 's can be quantitatively compared to the AFM measured $P_{j}$ 's, 


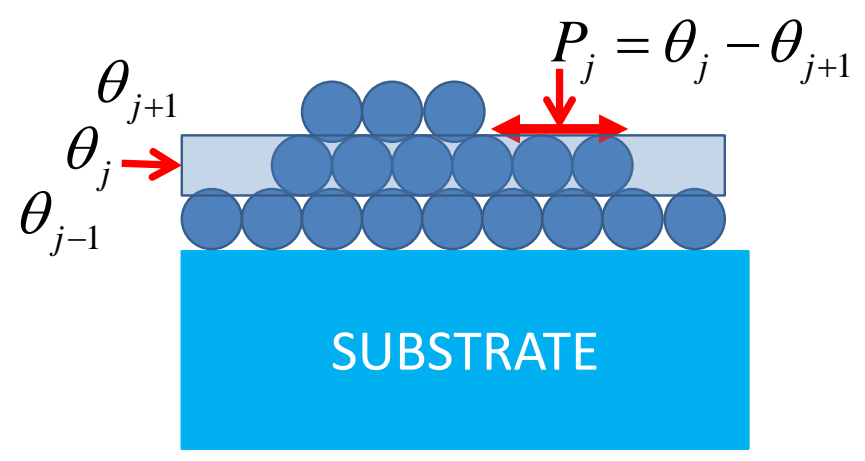

Figure 4.10: Illustration shows the relationship between the exposed surface fraction, $P_{j}$, and the coverage in the $j$-th layer, $\theta_{j}$, as $P_{j}=\theta_{j}-\theta_{j+1}$.

using the exposed surface fraction model relationships above.

\subsection{7 $\mathrm{X}$-ray reflectivity and AFM Quantitative Comparison}

Using the exposed surface fraction model relationships a quantitative comparison between AFM and X-ray measurements was made to gain insight into the low density $\mathrm{Ag}$ at the Si interface. For sample 80A, the modeled X-ray $\theta_{j}$ profile (upper panel of Figure 4.11) was used to compute the $\mathrm{X}$-ray $P_{j}$ profile $\left(P_{j}=\theta_{j}-\theta_{j+1}\right)$. As seen in Figure 4.11 (lower panel), the calculated $\mathrm{X}$-ray $P_{j}$ distribution (solid black line) was negative below $j<60$, corresponding to missing material below the surface. Since the AFM does not measure below the exposed surface, the measured AFM $P_{j}$ profile (red solid line) was rescaled (red dashed line) until it overlapped with the $\mathrm{X}$-ray $P_{j}$ profile (black solid line) and remarkably the exposed surface fractions matched identically for all $j>60$. The excellent match between the rescaled-AFM and the $\mathrm{X}$-ray $P_{j}$ profiles at the exposed surface suggest a high degree of accuracy of the density profiles derived from the X-ray reflectivity and, therefore, gives further support for the surprisingly low Ag density at the Ag/Si interface. 

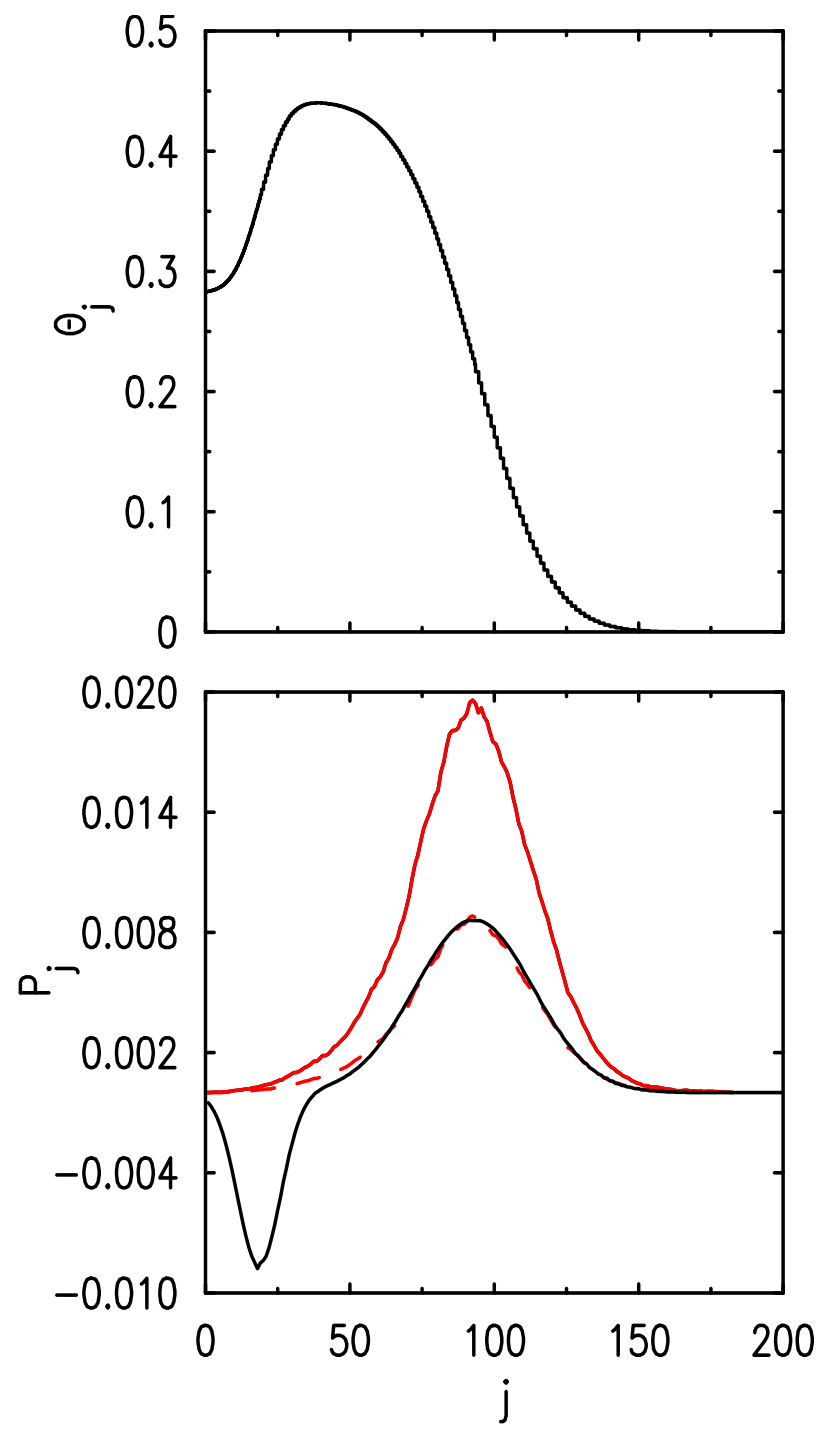

Figure 4.11: For sample 80A: a) upper panel shows the best fit $\mathrm{X}$-ray reflectivity modeled $\theta_{j}$ profile and $\mathrm{b}$ ) lower panel shows the exposed surface fraction $\left(P_{j}\right)$ as a function of each film layer $j$, where $j=0$ is the substrate. The $\mathrm{X}$-ray calculated $\mathrm{P}_{j}$ (black solid line), AFM (red solid line), and scaled AFM (red dashed line) are shown. Since the AFM does not see below the exposed surface $(j<60)$, as seen when the $\mathrm{X}$-ray $P_{j}$ distribution is less than 0 at the interface, the AFM was scaled such that the $P_{j}$ distributions overlapped. The agreement between the scaled AFM and the calculated $\mathrm{X}$-ray $P_{j}$ distribution for $j>60$ (exposed surface) is fairly remarkable. This comparison shows that the AFM and X-ray match at the surface, which leaves the buried interface to explain the lower than expected intensity of the $\mathrm{X}$-ray reflectivity and thus supports the observed lower interfacial density. 


\subsubsection{Discussion}

Fit parameters for samples 30A, 30U, and 80A recorded in Table 4.1, quantitatively show the deposition angle dependence on the film density, film height, and roughness. Most interesting in this work is not the decreasing of the density of the Ag film as a function of increasing deposition angle, but the even lower density at the $\mathrm{Ag} / \mathrm{Si}$ interface for all three samples. The lower interfacial density can be classified as missing material. Whether this missing material is in the form of trapped volumes, voids, or is open and connected to vacuum is not known.

This interfacial missing material below the exposed surface, $z<z^{\prime}$, can be quantified by directly integrating the $\rho_{b}$ profile as follows:

$$
\Theta_{M M}=\frac{1}{d_{111} * \rho_{b}^{A g}} \int_{z=0}^{z^{\prime}}\left[\rho_{b}^{1}-\rho_{b}(z)\right] d z
$$

which yields $1-4 \mathrm{ML}$ of missing material at the $\mathrm{Ag} / \mathrm{Si}$ interface, which can only be observed using $\mathrm{X}$-rays. When looking closely at the 80A AFM image in Figure 4.9a, one can see smaller Ag structures that are separated by 50nm and appear to be clumped together making up larger circular structures that resemble flower buds or cauliflower. Even though there is a noticeable surface morphology, there is a significant amount of missing material below the surface. When comparing the surface morphology to $\mathrm{MD}$ simulations of $\mathrm{Cu} / \mathrm{Cu}[3]$, there is a discrepancy in the length scales. The observed AFM length scales of 50nm are twice the size of the MD system size. The is not necessarily a fair comparison since one system is on $\mathrm{Ag} / \mathrm{Si}$ and the other is $\mathrm{Cu} / \mathrm{Cu}$, but it does show that these characteristic length scales for the surface morphological growth can be very large and have yet to be simulated. 


\subsubsection{Conclusion}

It was observed that the films got taller as the Ag density decreases and the roughness increases, as a function of increasing deposition angle. Also it was observed that there was missing material below the exposed surface of the Ag film as seen in the scattering length density profiles in Figure 4.12. The lower Ag interface was essential for obtaining a good fit to the $\mathrm{X}$-ray reflectivity data by decreasing the high $Q_{z}$ intensity. For all the deposition angles, $\rho_{b}$ profiles showed 1ML-4ML of missing material below the exposed surface at the $\mathrm{Ag} / \mathrm{Si}$ interface. Also, the film coverage obtained from this model was within error bars of the expected coverage, even though the coverage was not constrained in the model.

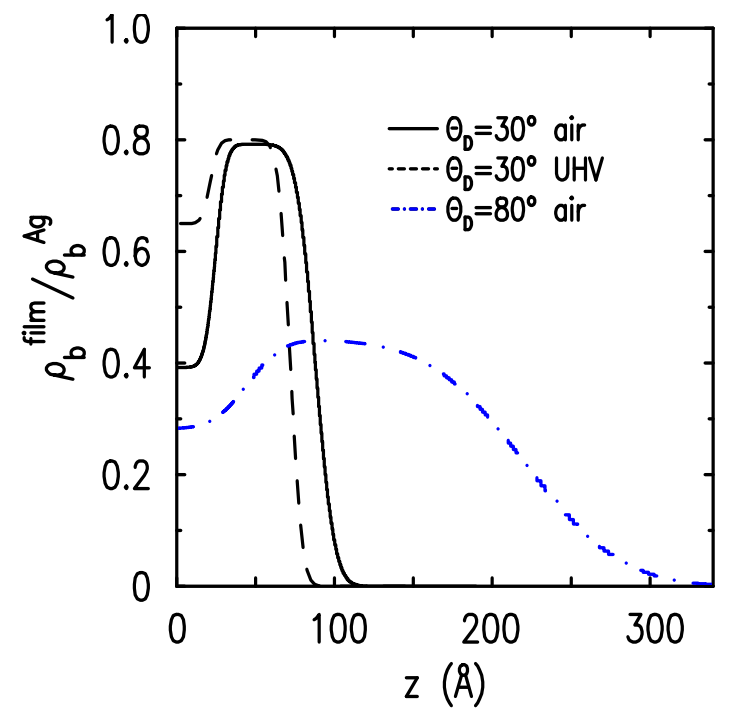

Figure 4.12: Comparison of the X-ray reflectivity best fit $\rho_{b}$ profiles are shown: sample 30A (solid line), 30U (dashed line), and 80U (blue dot-dashed line). As a function of increasing deposition angle: 1) the films become taller, 2) film roughness increase, and 3) film density decreases. All samples, for two deposition angles, show a lower density at the $\mathrm{Ag} / \mathrm{Si}$ interface, which correspond to a missing material of 1-4ML. 
What was learned about the film morphological structure? The average film $\rho_{b}^{1}$ was lower than the bulk $\mathrm{Ag} \rho_{b}^{A g}$, the film height was much larger than the deposited coverage, and there was adequate evidence for missing material below the exposed surface in all samples. Lateral profile information to address whether the structures were columnar, standing upright or tilted, or whether they were sponge like, porous, having voids buried below the exposed surface was not measured. However, due to the lower density, increased film heights, and the relatively large film roughness (of the order of the film thickness deposited) and since the AFM showed a budding type of morphology, I lean more towards a columnar type structural morphology as shown in the middle picture of Figure 4.13. Combining the columnar structure with the additional missing material below the exposed surface, one ends up with some type of a tapered structure near the substrate that increases in density up to the average film density and then grows rough, as seen in the right picture of Figure 4.13. It is difficult to say if these films are composed of tilted structures as seen by the GLAD technique and whether the missing material near the $\mathrm{Ag} / \mathrm{Si}$ interface is trapped or open to vacuum.

Also, our $\mathrm{Ag} / \mathrm{Si}$ studies did show less dense films with increasing deposition angle but most importantly, they revealed missing material below the surface which was crucial to fit the high $Q_{z} \mathrm{X}$-ray reflectivity data. This is not necessarily a fair comparison to the Amar simulations since they were not done for metal on semi-conductor, however, our comparison shows some of the complexity involved and the characteristic system size required in understanding components of thin film strains due to morphological structure and the requirement of missing material below the exposed surface. 


\section{LESS DENSE and MISSING MATERIAL NEAR INTERFACE}

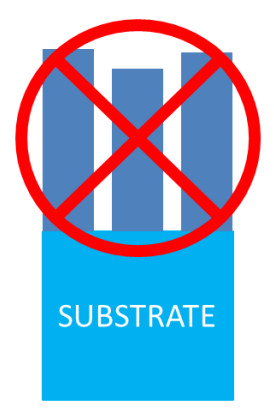

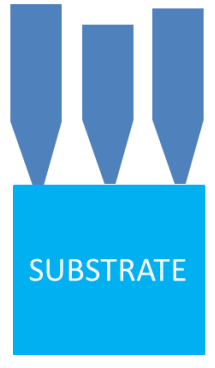

TAPERED

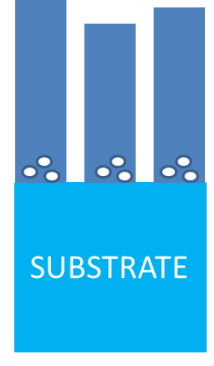

voIDs

Figure 4.13: A schematic summarizing the $\mathrm{X}$-ray reflectivity fits, which show taller structures with less density like the picture on the left, but it also shows missing material near the interface, more like picture on the right.

In previous works, the strain does not seem to vary with deposition angle[3], and with such large changes in the surface morphology, it seems unlikely that this would fully explain the film strains; especially when the missing material is of the order of a few ML and does not vary drastically with the deposition angle. It would appear that the missing material would be the more likely candidate for explaining the measured film strain as a function of deposition angle.

\section{4 $\operatorname{Ag}(001)$ homoepitaxial growth}

In situ deposition angle dependent $\mathrm{Ag} / \mathrm{Ag}(100)$ epitaxial growth for several coverages has been measured using $\mathrm{X}$-ray reflectivity in order to shed light on the vacancy mechanism responsible for film strain. Data taken over a large L-range $\left(Q_{z}=\frac{2 \pi L}{c}\right.$, where $\mathrm{c}$ is lattice spacing in the $\mathrm{z}$-direction, and $\mathrm{L}$ is the reciprocal lattice units) 
and plotted as a function of deposition angle for a given coverage resembles a "Pearl Necklace," as shown in Figure 4.14. It turns out that the anti-Bragg region (region in between Bragg peaks such as $\mathrm{L}=1.0$ and 3.0) is sensitive to the exposed surface that was shadowed by mesoscopic-structures, which scatters incoherently with the film. Reflectivity does not provide the sensitivity needed to quantify the lower density for homo-epitaxy, but it will be shown that there is little change in the strain profile below the exposed surface for varying surface roughness, or differing deposition angles. This constant strain below the surface irrespective of the surface roughness or angle of deposition leads one to infer that missing material (vacancies) below the surface is responsible for this strain.

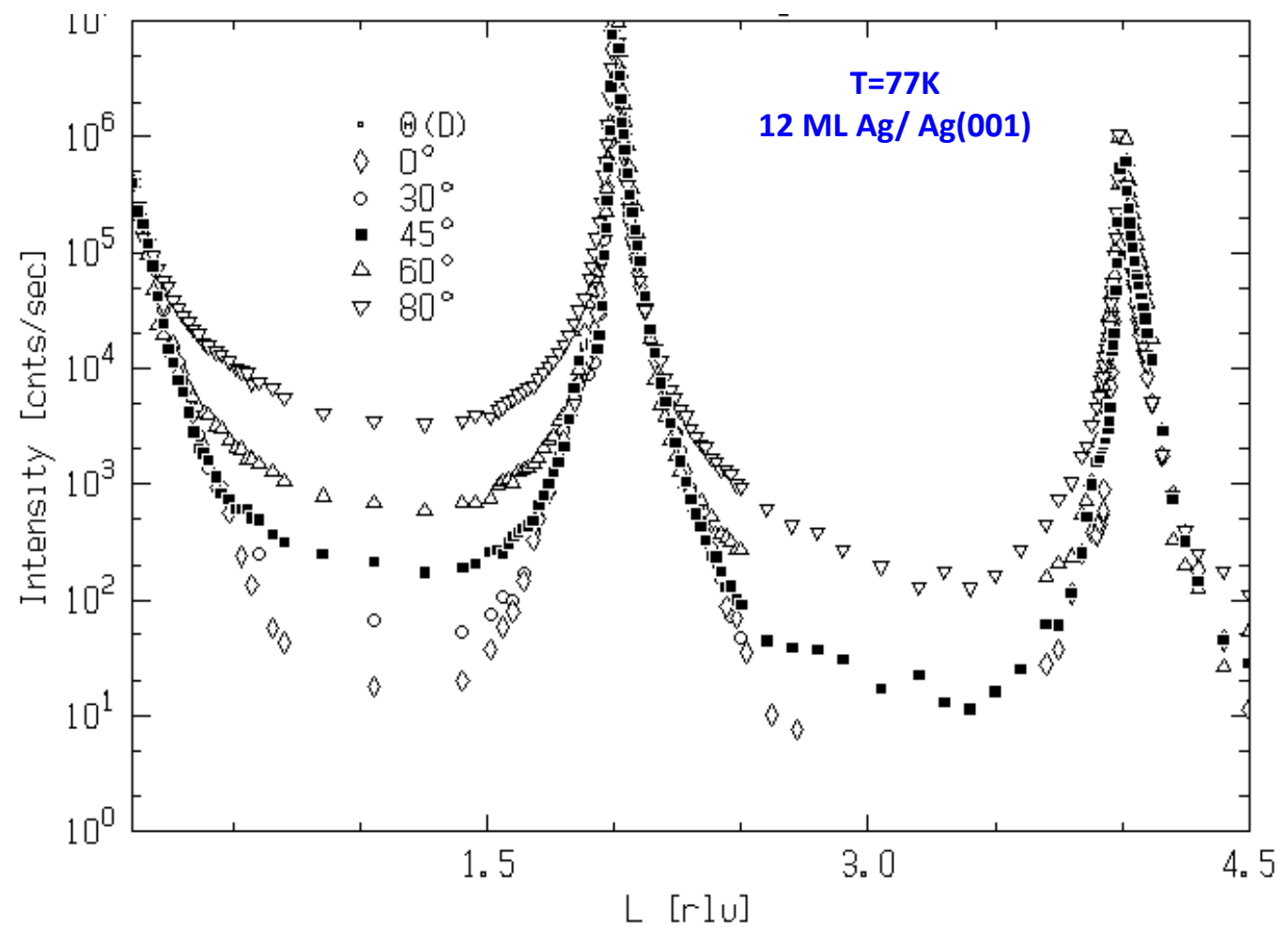

Figure 4.14: The deposition angle dependent XRR for $12 \mathrm{ML} \mathrm{Ag} / \mathrm{Ag}(100)$ resembles a "Pearl Necklace" over a wide range of Q. 
Pyramidal multilayer and layer-by-layer reflectivity models were not able to fit the data. The models either fit the Bragg regions or the anti-Bragg regions, but not both. Also, the models yielded coverages that were too low by more than $50 \%$ of the expected coverage. In an attempt to model and understand the data, these models were scrapped for a very basic film model with some embellishments. A sharp transition in the buried interface film density would also reproduce intensity at the anti-Bragg, as a function of deposition angle. However, this model was also scraped due to the fact that a sharp density transition also leads to excessive ringing which was not observed in any of the data, and the ringing could not be dampened out. Unsuccessful attempts to dampen out the density ringing were: 1) very large surface roughness, 2) very long and smooth density transition from the abrupt density change to the surface, 3) incorporating a strain profile that also had very long transition widths as a function of the film height.

\subsubsection{Ag/Ag experimental details}

The surface preparation, growth, and x-ray scattering measurements were carried out in ultra-high vacuum $\left(P \sim 10^{-10}\right.$ Torr $)$ using the in situ surface scattering instrument at the 6ID-C $\mu$ CAT beamline located at the Advanced Photon Source, shown in Figure 4.15. The clean surface was prepared by repeated cycles of ion sputtering and high-temperature annealing. Auger electron spectroscopy (AES) showed no trace of contaminants on the clean surface within AES sensitivity.

The clean surface was prepared for each deposition angle only. Then reflectivity for each of the coverages of 6ML, 12ML, and 24ML (as appropriate) was measured. For the given deposition angle, the coverages were cumulative, meaning that to obtain the 
next coverage, material was deposited on top of the existing coverage, excluding the first $6 \mathrm{ML}$ which was put directly on a clean surface. For example, to obtain $12 \mathrm{ML}$, we added 6ML to the existing 6ML film. Previous experiments[21] have shown that there is no difference in $\mathrm{X}$-ray reflectivity measurements from depositing homoepitaxial Ag films cumulatively, or just all at once (i.e. cleaning the surface and depositing entire coverage).

\subsection{2 $\mathrm{X}$-ray reflectivity results: Overview}

Qualitative observation from the "Pearl Necklace" plot, shown in Figure 4.14, show that most of the changes are occurring in the anti-Bragg region. Plotting anti-Bragg intensity at $\mathrm{L}=1.05$ (normalized by the clean surface reflectivity) vs coverage as a function of deposition angle is shown in Figure4.16. The anti-Bragg intensity sets in early on in the deposition and does not vary with coverage. Also, the anti-Bragg intensity decreases orders of magnitude versus deposition angle. Furthermore, it is independent of coverage and indirectly related and extremely sensitive to the deposition angle.

The anti-Bragg region depends on the deposition angle and not on the coverage. Another interesting dependence was characterized by plotting the anti-Bragg intensity vs the deposition angle, as seen in Figure 4.16. An exponential function with the cosine of the deposition angle, $\Theta_{D}$, in the exponent was found to fit very nicely to the data, as shown in Figure 4.17. This anti-Bragg intensity dependence is believed to come from superposition of incoherent substrate scattering from the shadowing of the clean substrate mesoscopic structures shown in Figure 4.18. This is the fraction of the clean surface that did not see any deposition flux. 


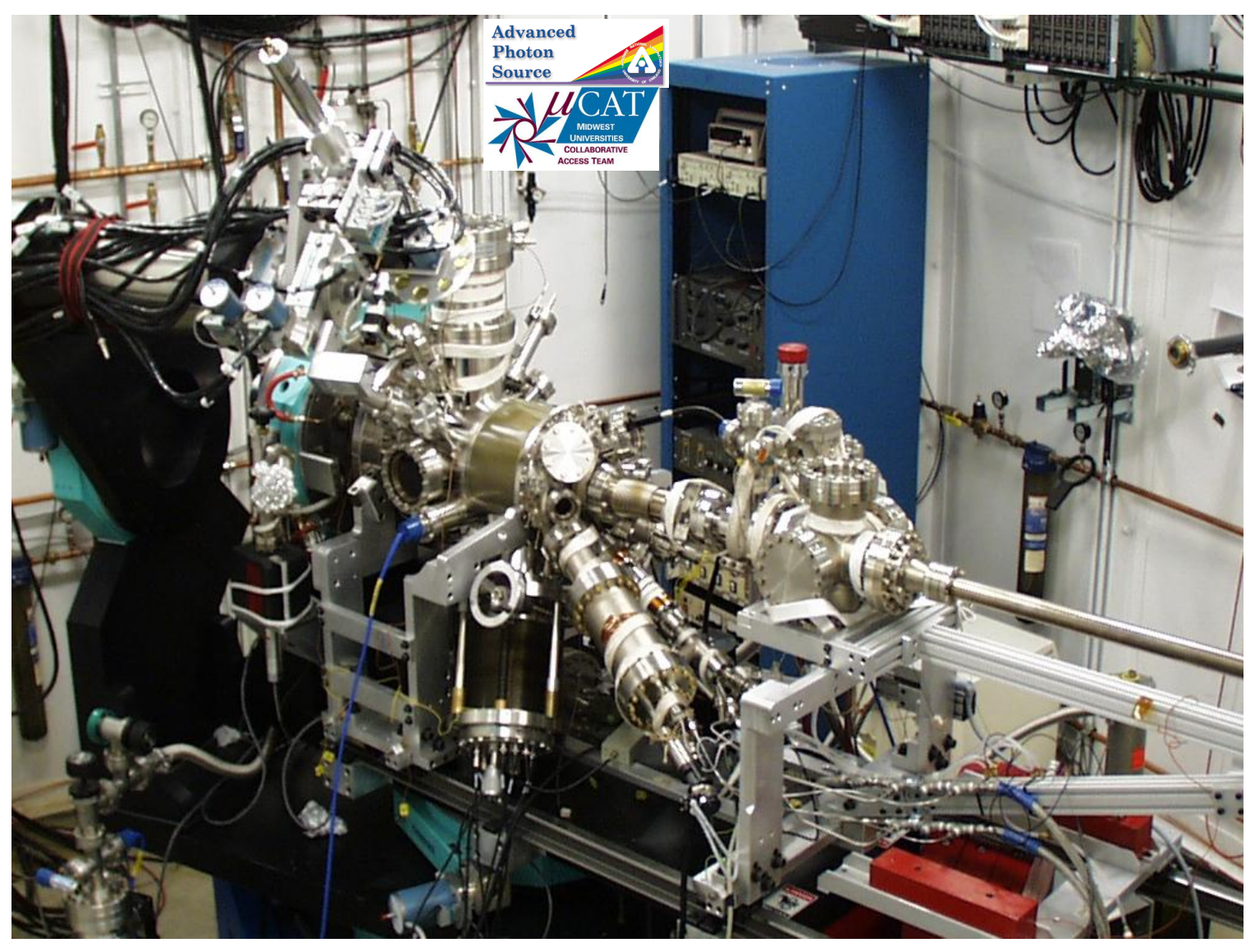

Figure 4.15: State of the art PSIC in situ UHV surface science chamber located at the Argonne National Laboratory Advance Photon Source 6 ID-C covers $\sim 1 \pi$ steradians (solid angle) of reciprocal space using a large Be window. In situ surface science tools included on the chamber are: 1) an Auger electron spectroscopy (AES), 2) a low-energy election diffraction (LEED), 3) an EPI and 4) Omicron evaporators, and 5) $\mathrm{a} \mathrm{Ar}^{+}$source used with 6) an ionization sputter gun. A separate attached loadlock chamber has the ability to hold 8 samples and uses an in-situ magnetic coupling transfer arm for placing samples into the main chamber. 


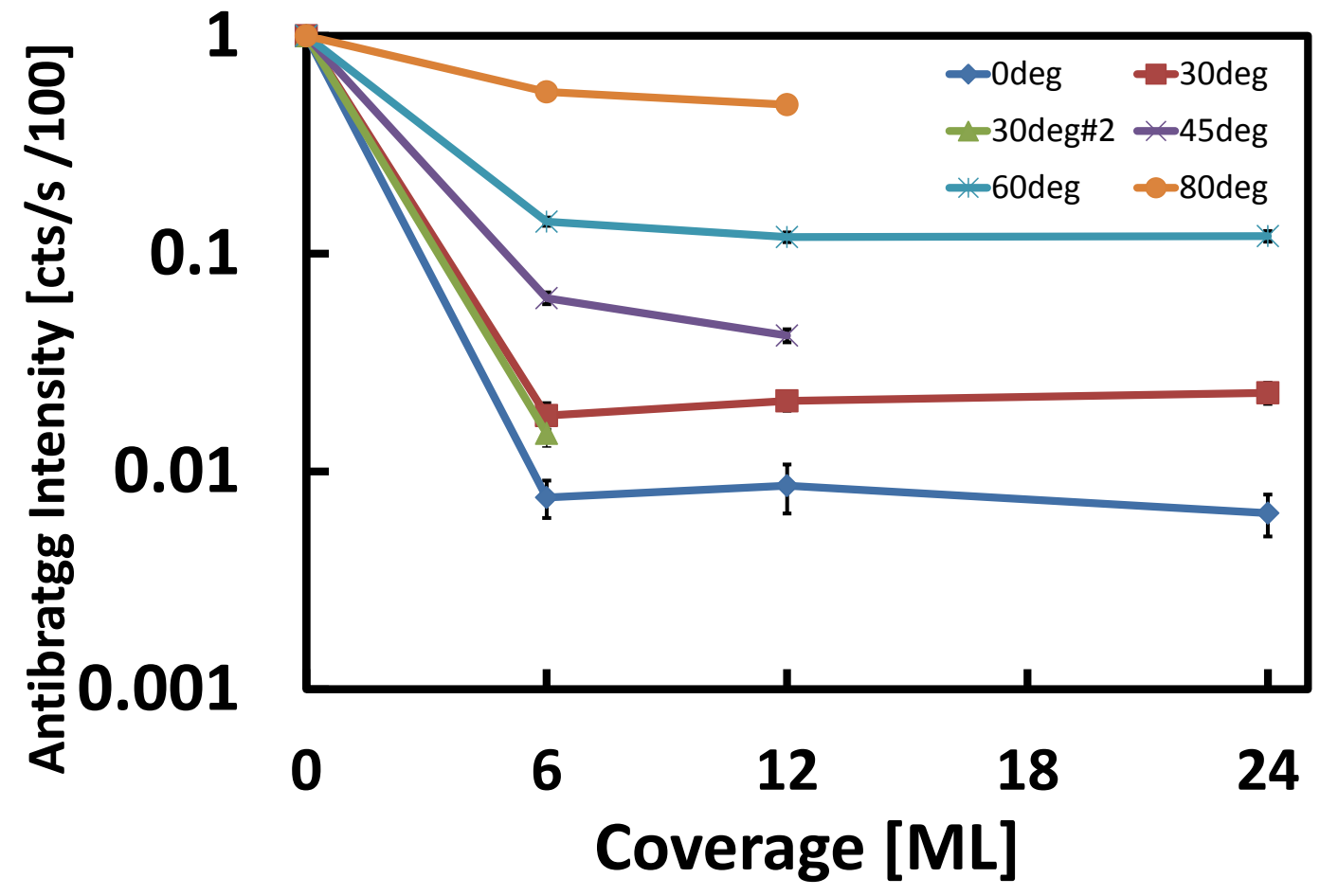

Figure 4.16: Anti-Bragg intensity vs coverage for different deposition angles. 


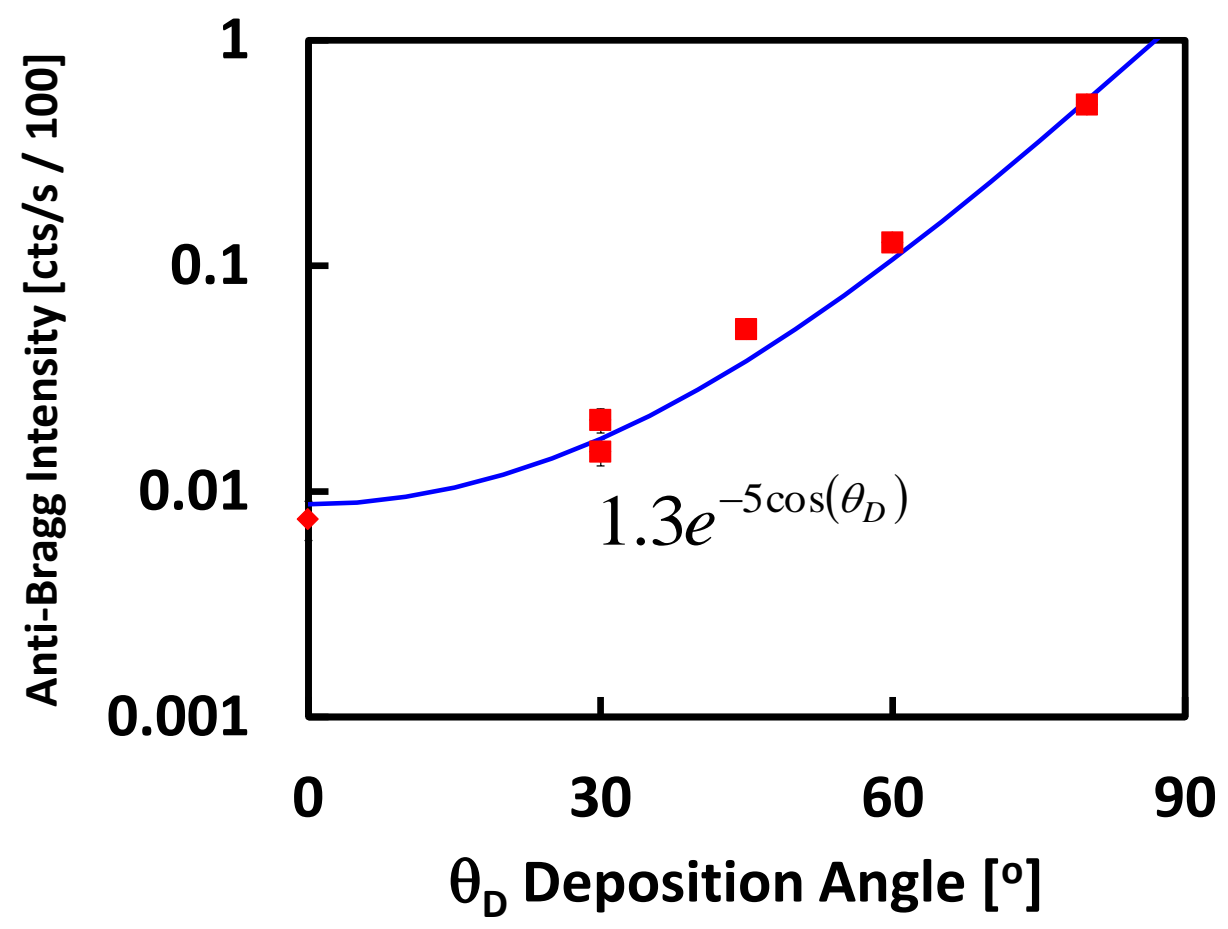

Figure 4.17: Anti-Bragg intensity vs deposition angle dependence. It is interesting that is fits to $\cos \left(\Theta_{D}\right)$ in the exponent of an exponential. 


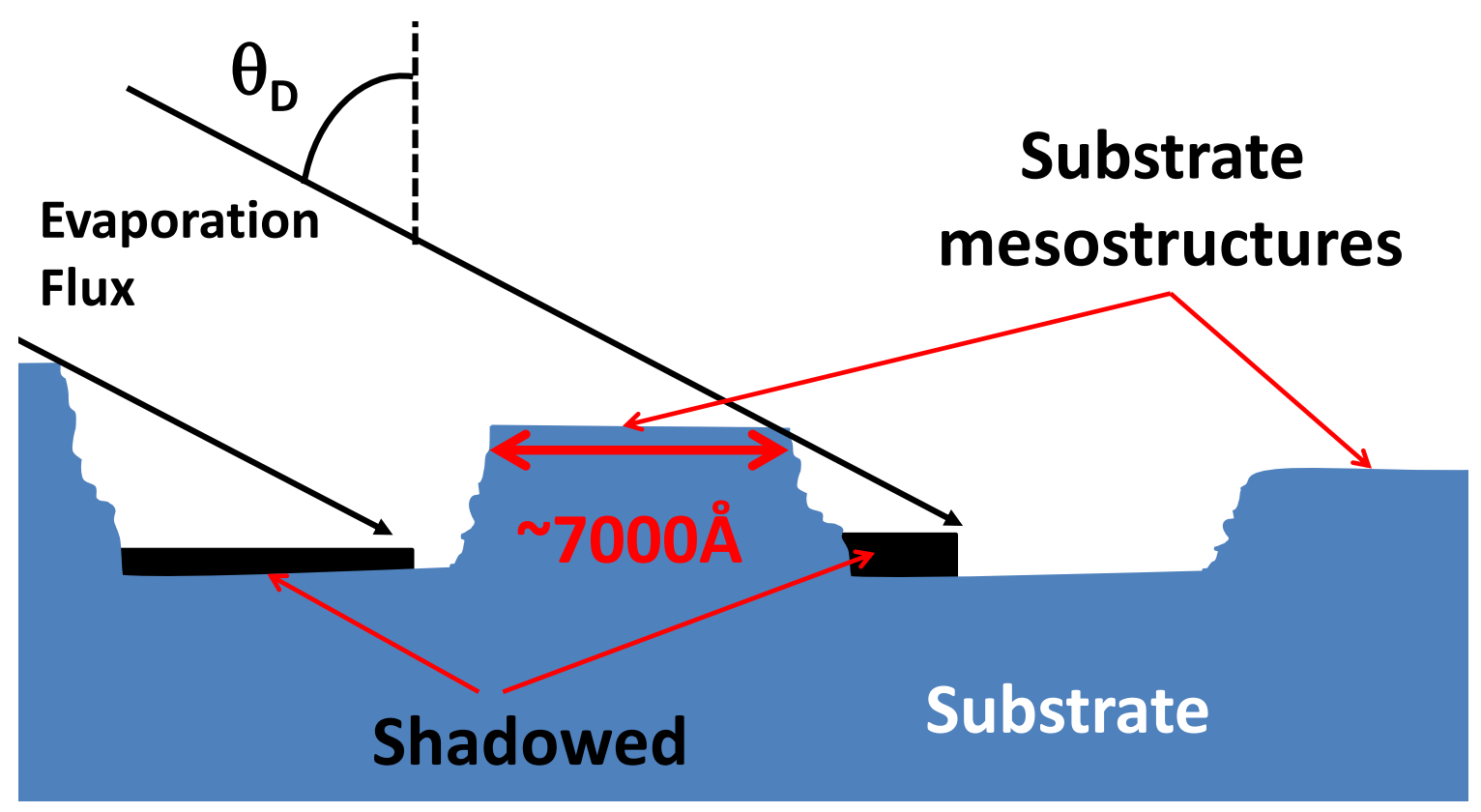

Figure 4.18: This illustration shows the lateral length scale of mesoscopic features on the clean $\mathrm{Ag}(100)$ substrate measured from the domain broadening as measured by low $\mathrm{Q}$ reflectivity. This shadowed region is believed to be responsible for the deposition angle anti-Bragg intensity dependence due to the superposition of incoherent substrate scattering from the shadowed substrate piece that never saw deposition flux due to large scale mesoscopic substrate structures. 


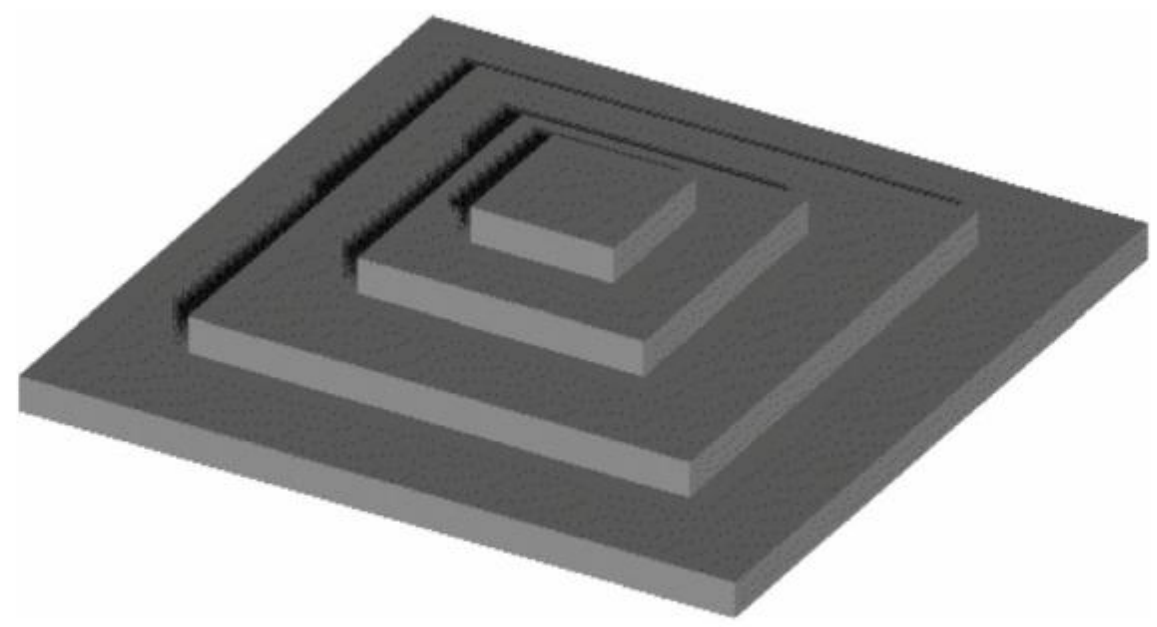

Figure 4.19: Schematic illustration shows the pyramidal geometry used to successfully describe noble metal (111) structures. A fingerprint for 3D morphological structures in $\mathrm{X}$-ray reflectivity measurements are thin film oscillations at low Q. From Ref. [24]

\subsubsection{X-ray reflectivity embellished model}

Modeling with several thin film reflectivity models did not fit the data and they also gave coverages that were half of the expected coverage. The layer-by-layer model is simply a film sitting on a substrate that exhibits statistical Gaussian surface roughness. The multilayer pyramidal model uses a pyramidal geometry, shown in Figure 4.19, that leads to non-Gaussian distribution of terrace heights[24] with a fingerprint of low Q grazing incidence thin film oscillations, which is not seen in the data.

We have embellished the layer-by-layer model by adding in a factor, $f$, which approximates mounding (see Figure 4.20) in order to test sensitivity to buried interfacial missing material. Also added was a phase factor which allows the first layer of the film to have a slightly different lattice parameter than the bulk of the film.

The reflectivity was calculated using Equation 4.7, by squaring the sum of two amplitudes, the substrate plus the film, where $V_{0}$ is the substrate roughness, $V_{f}$ is the 


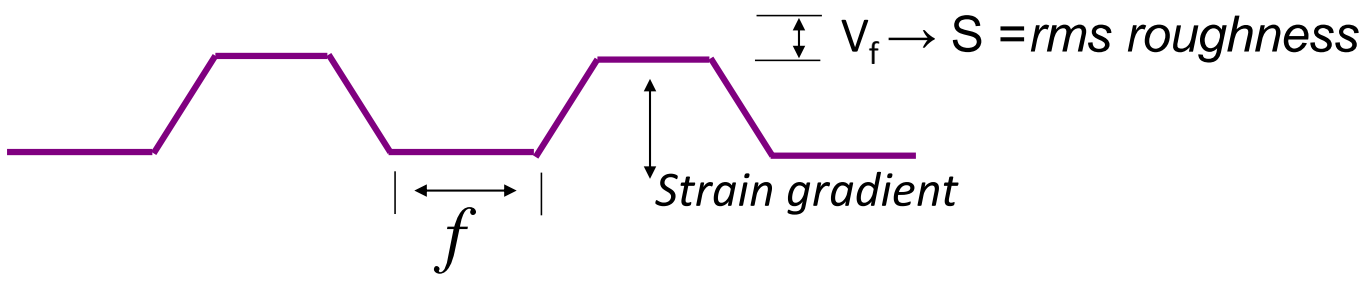

Figure 4.20: Embellished simple model used to understand the anti-Bragg region. Two terms were added to the film, f, which approximates mounding and a strain gradient.

film surface roughness, $d_{\text {substrate }}$ is the substrate lattice spacing, $d_{f i l m}$ is the average film lattice spacing. For a $1 \%$ compressive film strain, $\left(d_{\text {film }}-d_{\text {substrate }}\right) / d_{\text {substrate }}$, gives a noticeable shift of the reflectivity scattering to high $\mathrm{Q}$, see bottom plot of Figure 4.21 .

$$
|A|^{2}=\left|V_{0}\right|^{2}\left|\frac{1}{1-e^{-i Q d_{\text {substrate }}}}+\left[\mathbf{f} * \mathbf{e}^{\mathbf{i} \mathbf{Q D _ { \text { off } }}}\right] \frac{V_{f}-1}{1-e^{-i Q d_{\text {film }}}}\right|^{2}
$$

In spite of the crudeness of the model, it was able to match the anti-Bragg intensities, as shown in Figure 4.21. The model oscillations are too large, but the correct ratio of anti-Bragg intensities to Bragg intensities were obtained. 

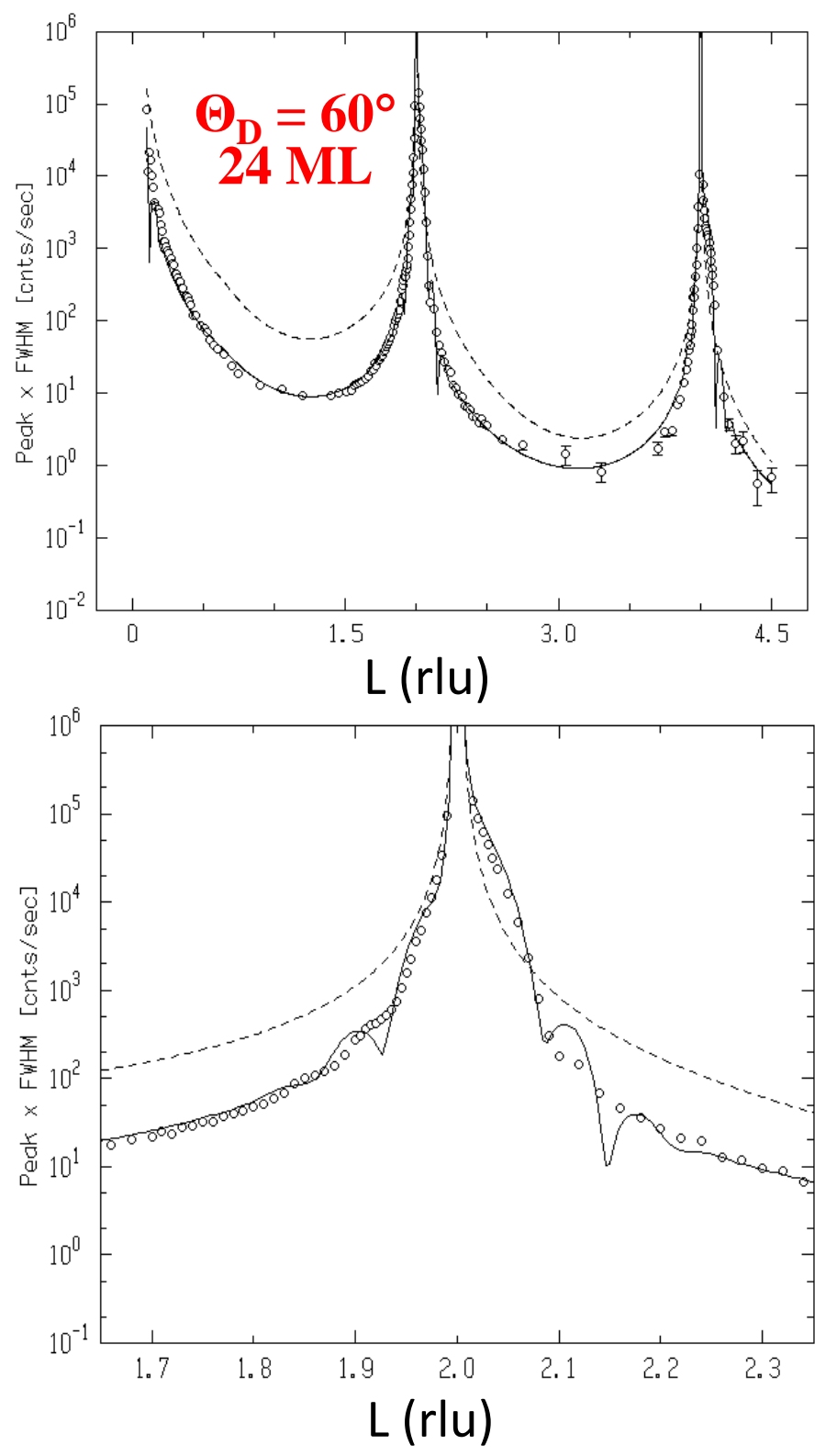

Figure 4.21: The embellished model (solid line) fits the anti-Bragg region of the $\Theta_{D}=60^{\circ} 24 \mathrm{ML}$ data (open circles) very well, but still generates intense oscillations that cannot be dampened with a very large surface roughness. This large oscillation intensities in the model are not seen in the data at $\mathrm{L}=1.9,2.1$ and 2.18. The bottom plot is zoomed in on the $\mathrm{L}=2$ region.

The $f$ factor vs coverage, does not depend on coverage but on deposition angle. If 
this model were correct, it would suggest that the morphology at the lower interface is set early on during deposition and is maintained throughout for thicker coverages as shown in the plots of fitting parameters versus coverage as a function of deposition angle (Figure 4.22).

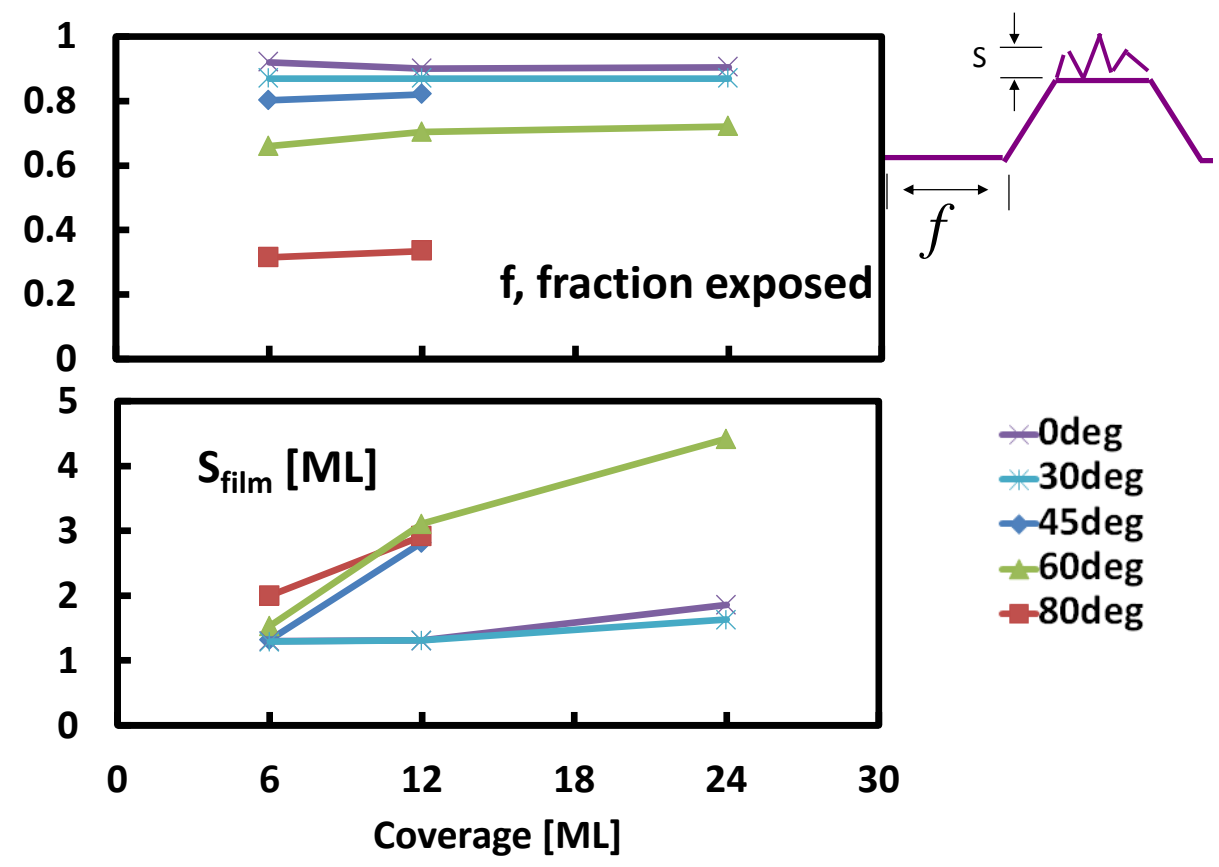

Figure 4.22: Plotted are the embellished model fit parameters: a) $f$, exposed surface fraction (top plot), and b) S, rms surface roughness (bottom plot) versus coverage as a function of deposition angle. The exposed surface fraction is a constant for a given deposition angle, suggesting early set in of a lower density below the exposed surface. It does increase with increasing deposition angle due to increased shadowing at oblique deposition angles. The roughness increases with both coverage and deposition angle, as expected. 


\subsubsection{Incoherent Shadowed Substrate Model}

The issue has always been trying to understand why there is such a large increase in the $\mathrm{Ag} / \mathrm{Ag}(001)$ reflectivity anti-Bragg ( $\mathrm{L}=1$ and 3$)$ intensity as the deposition angle increases. The specular intensity should drop to 0 at the anti-Bragg for a sufficiently rough film, $A_{\text {substrate }}=-A_{\text {film }}$ at $\mathrm{L}=1(Q d=\pi)$, which is not what was observed in our experiments. Using the embellished model, the anti-Bragg intensity could be explained with an abrupt density transition at the buried interface, however, this led to excessive ringing, which is again not observed in the data, see Figure 4.21. This led to calculating reflectivity from different types of surface, strain, and disorder distributions in order to dampen the large oscillations caused by the abrupt density transition of the embellished model, yielding the anti-Bragg intensity, but missing other key features of the data due to excessive ringing. Thus the embellished model and any derivatives were not going to work due to the abrupt density transition.

A model was needed that allowed for an increase in the anti-Bragg intensity, but did not cause any other additional oscillation ringing. The revelation came from finding a way to add intensity to the anti-Bragg without introducing the ringing from an abrupt density change. The obvious answer was to add in a superposition of incoherent substrate scattering to the existing coherent film plus substrate modeled intensity. It is believed that the incoherent scattering is coming from the exposed surface fraction, $f_{h}$, that was being shadowed from mesoscopic substrate structures during deposition. This means that these shadowed regions never covered with deposition flux. 
The calculated reflectivity is modified by adding the incoherent surface fraction $f_{h}$, as follows:

$$
I_{\text {modeled }}=f_{h} * I_{\text {sub }}+\left(1-f_{h}\right) * I_{\text {sub }+ \text { film }}
$$

where $I_{\text {sub }}=A_{\text {sub }} * A_{\text {sub }}^{*}$ is the substrate intensity, and

$$
I_{\text {sub }+ \text { film }}=A_{\text {sub }+ \text { film }} A_{\text {sub }+ \text { film }}^{*}
$$

where $A_{\text {sub film }}=A_{\text {sub }}+A_{\text {film }}$, is the intensity coming from the square of the sum of the substrate plus film amplitudes.

This model worked quite well by allowing the fitting of the anti-Bragg regions without introducing any additional ringing. The anti-Bragg intensity appears to be coming from regions of the substrate that were shadowed from Mesozoic Ag substrate structures that never received any deposition flux.

Using the features from the data, a simple model was derived from a binomial distribution for the film density ( $F_{j}$ distribution shown in Figure 4.23), a modified binomial distribution for the film strain $\left(\Delta d_{j}\right.$ as seen in Figure 4.24), and a linear distribution for the film disorder $\left(u_{j}^{2}\right.$ as shown in Figure 4.25). This model works to fit all the features of the coverage dependent $\mathrm{X}$-ray reflectivity data as a function of deposition angle. The following plots show the distributions used.

$$
A_{\text {film }}=\sum_{j=1}^{\infty} F_{j} e^{-i Q_{z} z_{j}} e^{-Q_{z}^{2} u_{j}^{2}}
$$

where $j$ is the index number for an atomic layer in the $z$-direction, perpendicular to the surface $(z=j d), d_{j}$ is the average inter-atomic layer spacing for the $j$-th 
layer, and $u_{j}^{2}$ is the statistical variation from the average of all the atoms in the $j$-th layer. $F_{j}$ is choosen to be a binomial distribution[?] where $\bar{N}$ is the average film height, and $S$ is the rms roughness of the film about height $\bar{N}$. Also, the disorder was choosen to have a Marcovian disorder, where every layer on top builds from the previous layer disorder, $u_{j}^{2}=\alpha j$, where $\alpha$ is the linear slope disorder parameter. A quadratic increase in the disorder was used, but it was indistinguishable from the linear disorder, so we choose the simpler model.

There is enough sensitivity with the strain model to determine that the surface strain does not:

- decay from the surface strain back in an exponential form to the substrate (i.e. $\left.N_{D 1}=0\right)$,

- surface strain does not go straight back to the substrate in a homegeneous fashion (i.e. $\Delta d_{1}=-1 \%$ ).

Therefore a two transition strain model was choosen. 


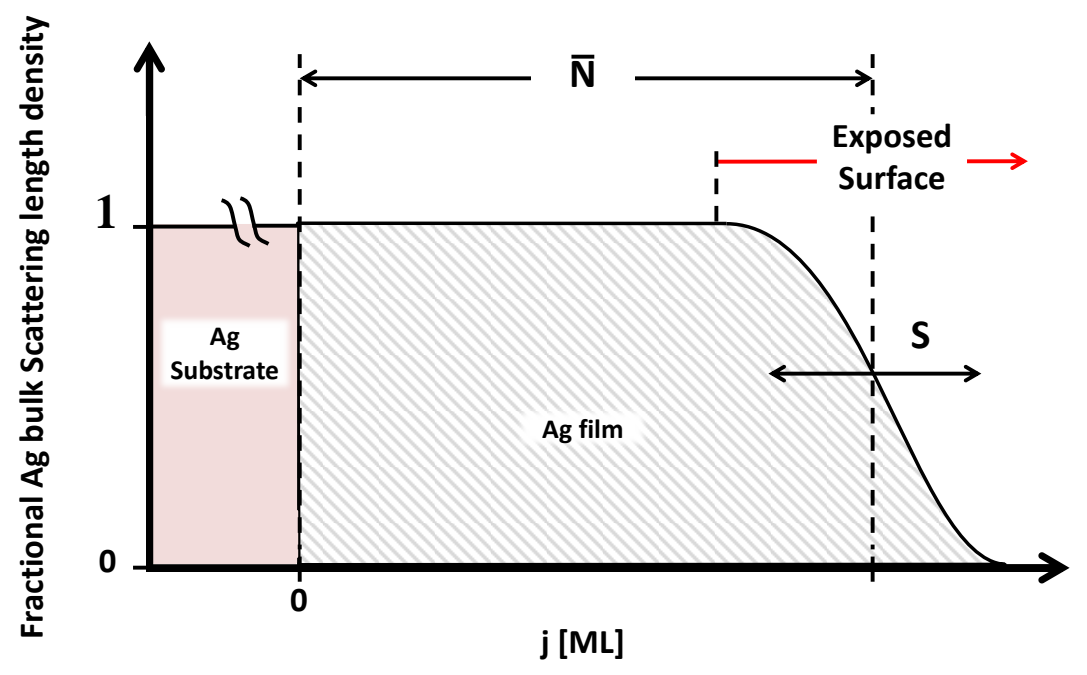

Figure 4.23: Density profile $\left(F_{j}\right)$ is constructed from a simple binomial roughness distribution, with an average film thickness of $\bar{N}$ with a rms surface roughness of S.

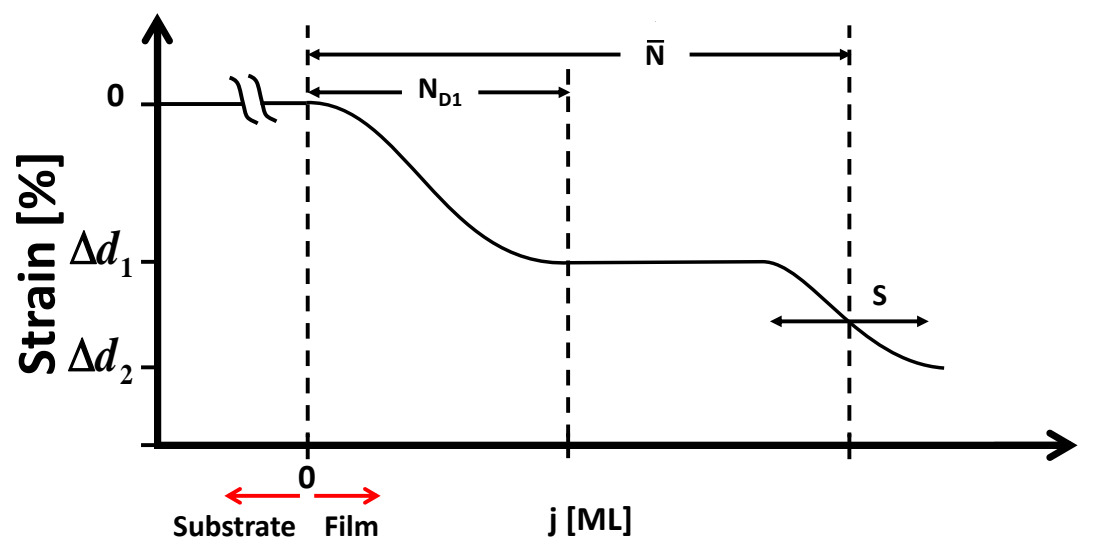

Figure 4.24: The average film lattice strain in a given $\mathrm{j}$-th layer is allowed to vary using the strain profile $\left(\Delta d_{j}\right)$ given above. The $\Delta d_{j}$ profile starts from the substrate with 0 strain and smoothly varies to $\Delta d_{1}$ using a sinusodial function over the distance $N_{D 1}$. The distribution continues to vary to $\Delta d_{2}$, near the exposed surface, following a binomial distribution where $\bar{N}$ and $\mathrm{S}$ are constrained to the density profile values. 


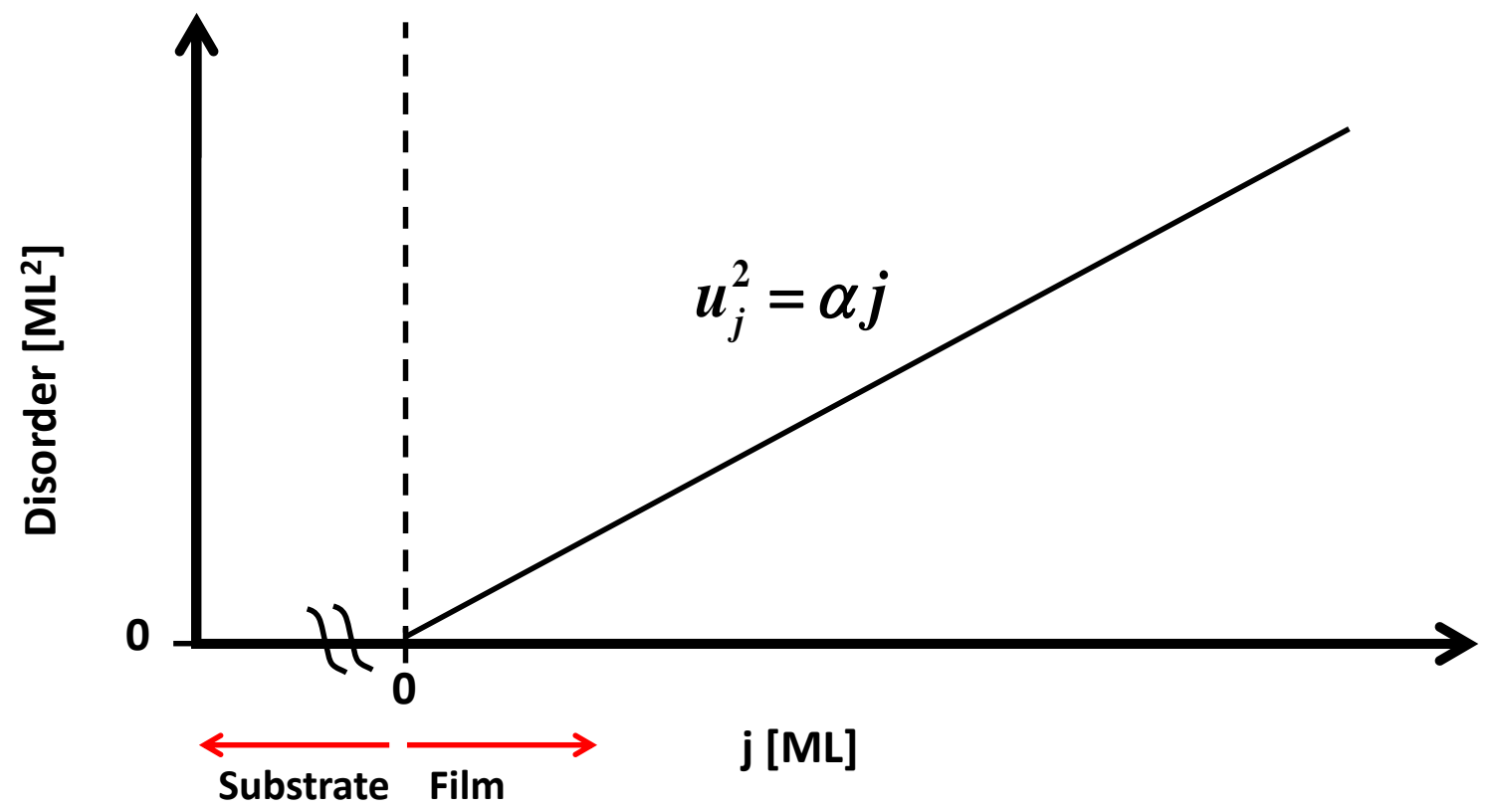

Figure 4.25: The rms roughness of the average film lattice parameter is allowed to vary using the disorder profile $\left(u_{j}^{2}\right)$ given above. The $u_{j}^{2}$ profile simply follows a Marcovian disorder, where each successive layer in $\mathrm{j}$ builds on top of the previous layer, therefore to first order, the disorder can be approximated as simply increasing linear with $\mathrm{j}$ with a slope of $\alpha$. The distribution continually increases until the density profile goes to 0 .

Initial parameters were estimated using the data. For deposition angles below $60^{\circ}$ there was a noticeable thin film oscillation which sets the film height $\bar{N}$. Next, the film roughness $S$ could be set using the slope of the low Q slope. Starting values for the strain distribution are as follows:

$$
N_{D 1}=12 M L
$$




$$
\Delta d_{1}=-1 \%
$$

$\Delta d_{2}$ was fixed to $-1 \%$ for all data sets. There was not a lot of sensitivity to the ending surface strain because the density near the very top film layers goes to 0 . Starting values for the film disorder was $\alpha=2 E-3$.

For a given deposition angle, I simultaneously fit the 6ML, 12ML, and $24 \mathrm{ML}$ data, requiring that the following parameters not vary as a function of coverage: a) $N_{D 1}$, b) $\Delta d_{1}$, and c) $\alpha$. The following parameters were allowed to refine using least square fitting: $\bar{N}, N_{D 1}$, and $\alpha$. The surface roughness $S$, had to be changed manually because the binomial distribution requires the total possible number of trials to be an integer. The only parameter allowed to be different for a given deposition angle was the average film height, $\bar{N}$, and roughness $S$. This significantly improved the sensitivity to the strain profile and disorder.

The incoherent substrate shadowing model fits, over a large range of deposition angles and coverage $\left(\Theta_{D}=0^{\circ}, 30^{\circ}, 45^{\circ}, 60^{\circ}\right.$ and $80^{\circ}, 6 \mathrm{ML}, 12 \mathrm{ML}$, and $24 \mathrm{ML}$ (were applicable)), all the salient features of the data: oscillation spacing, strain around bragg peaks, and the anti-Bragg intensity, as shown in Figures 4.26 - 4.30. 

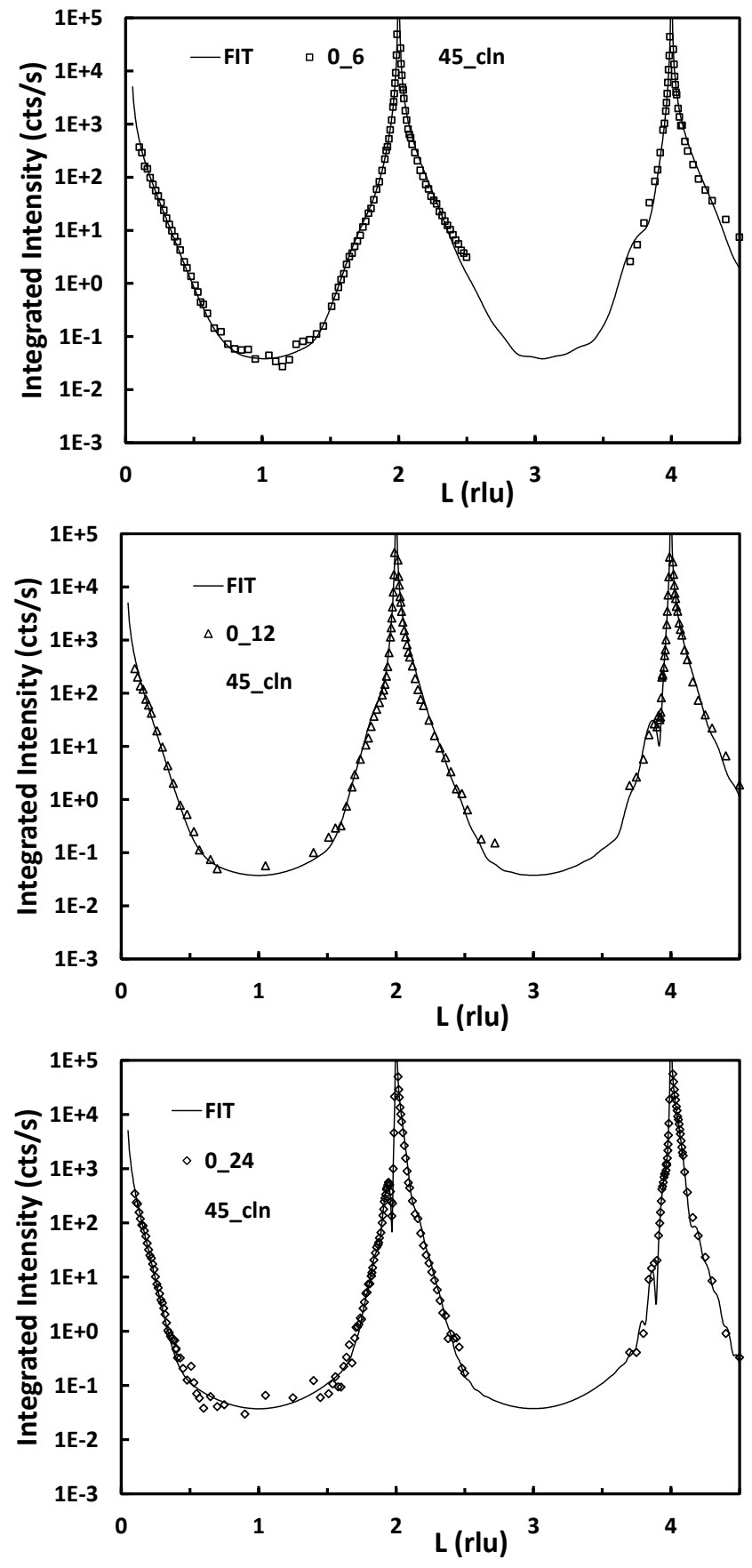

Figure 4.26: The $\Theta_{D}=0^{\circ}$ data (open symbols) for 6ML (top plot), 12ML (middle plot), 24ML (bottom plot) is plotted with the incoherent substrate shadowing model best fit (black solid line). This model provides a good fit to all the salient features of the three coverages: low $\mathrm{Q}$ range, anti-Bragg, thin film oscillation spacing, and the strain near the Bragg peaks. 

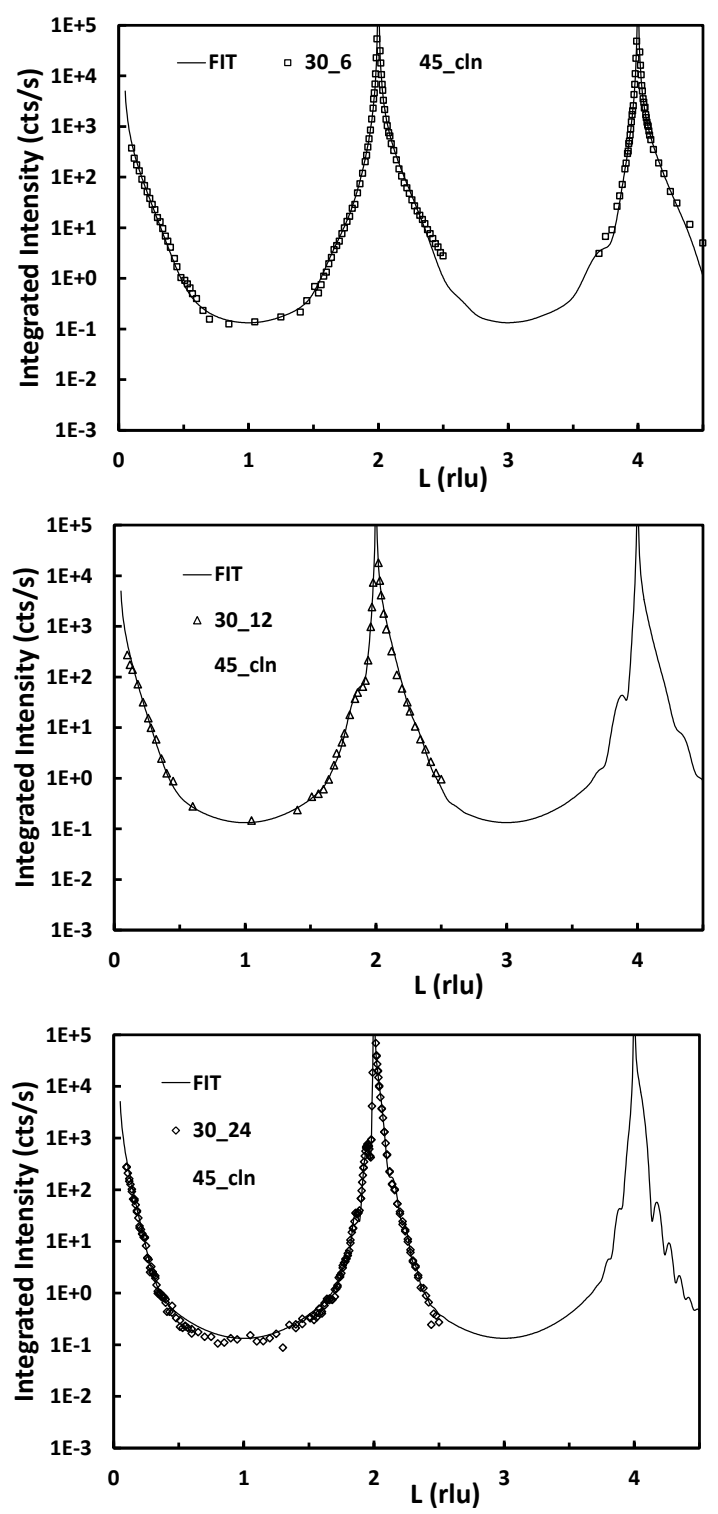

Figure 4.27: The $\Theta_{D}=30^{\circ}$ data (open symbols) for 6ML (top plot), 12ML (middle plot), 24ML (bottom plot) is plotted with the incoherent substrate shadowing model best fit (black solid line). This model provides a good fit to all the salient features of the three coverages: low $\mathrm{Q}$ range, anti-Bragg, thin film oscillation spacing, and the strain near the Bragg peaks. Unfortunately, $\mathrm{L}=4$ data was not recorded for the $12 \mathrm{ML}$ and $24 \mathrm{ML}$ data sets, but the fitted lines are still shown going out to $\mathrm{L}=4.5$. 

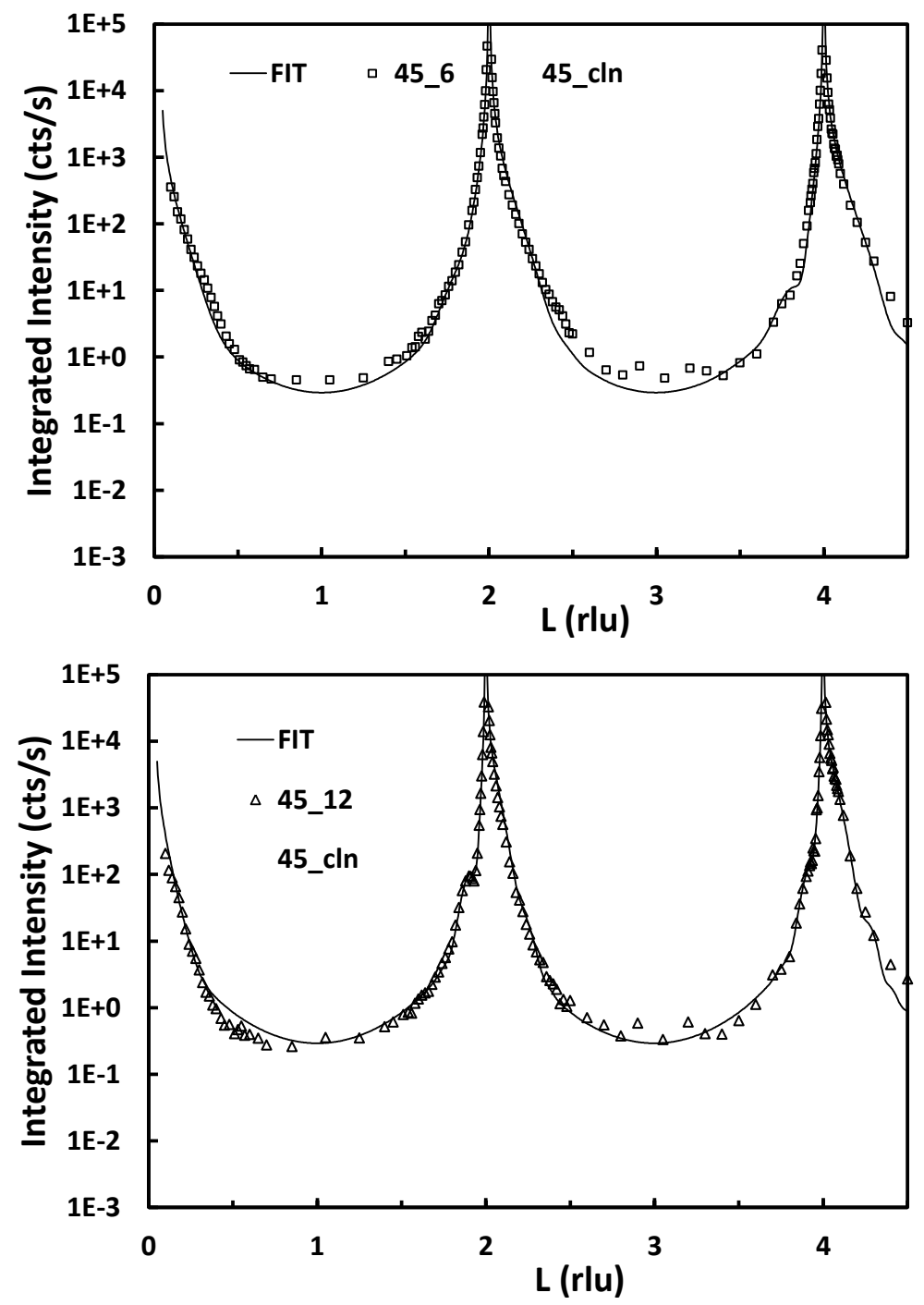

Figure 4.28: The $\Theta_{D}=45^{\circ}$ data (open symbols) for 6ML (top plot), 12ML (bottom plot), is plotted with the incoherent substrate shadowing model best fit (black solid line). This model provides a good fit to all the salient features of the three coverages: low $Q$ range, anti-Bragg, thin film oscillation spacing, and the strain near the Bragg peaks. 

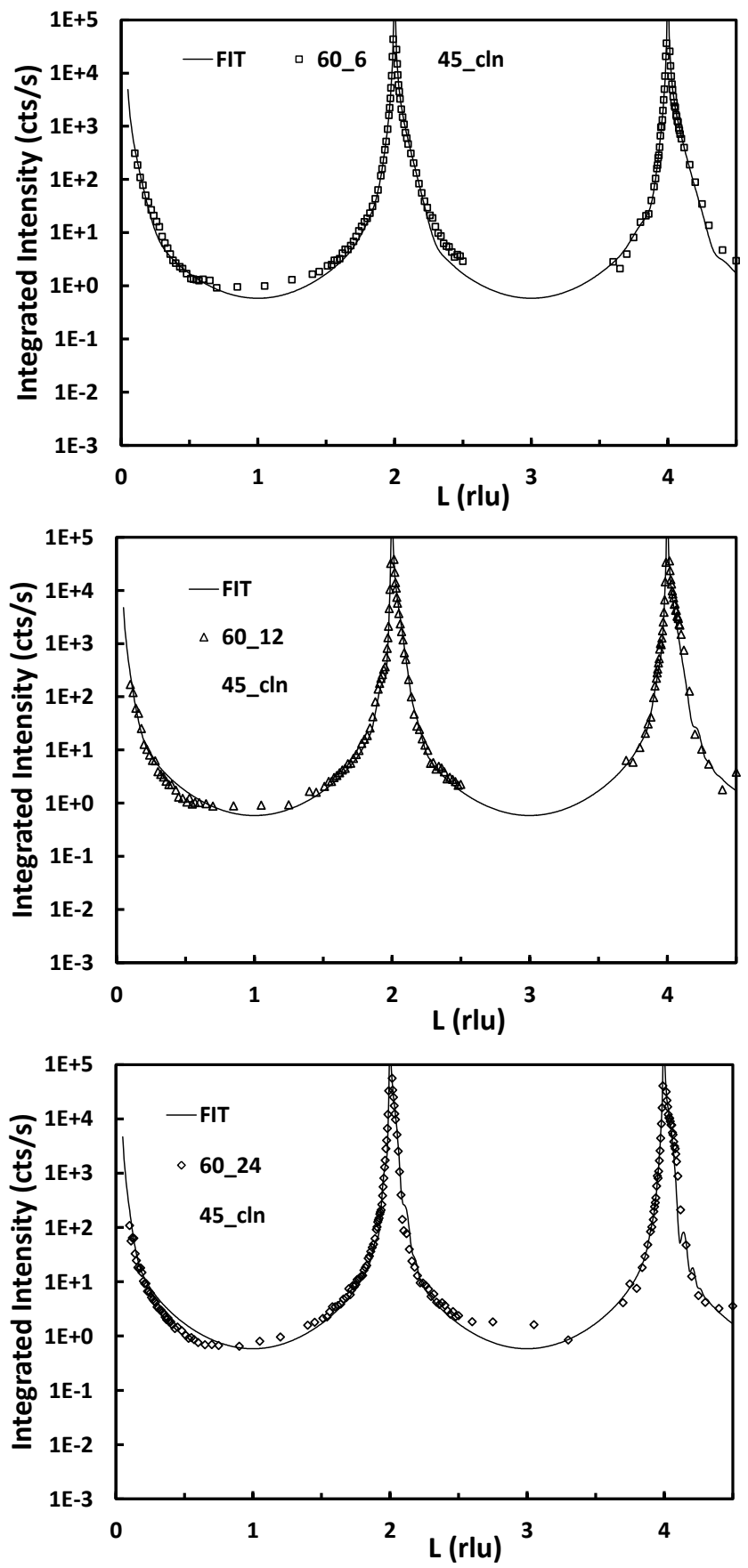

Figure 4.29: The $\Theta_{D}=60^{\circ}$ data (open symbols) for 6ML (top plot), 12ML (middle plot), 24ML (bottom plot) is plotted with the incoherent substrate shadowing model best fit (black solid line). This model provides a good fit to all the salient features of the three coverages: low $\mathrm{Q}$ range, anti-Bragg, thin film oscillation spacing, and the strain near the Bragg peaks. 

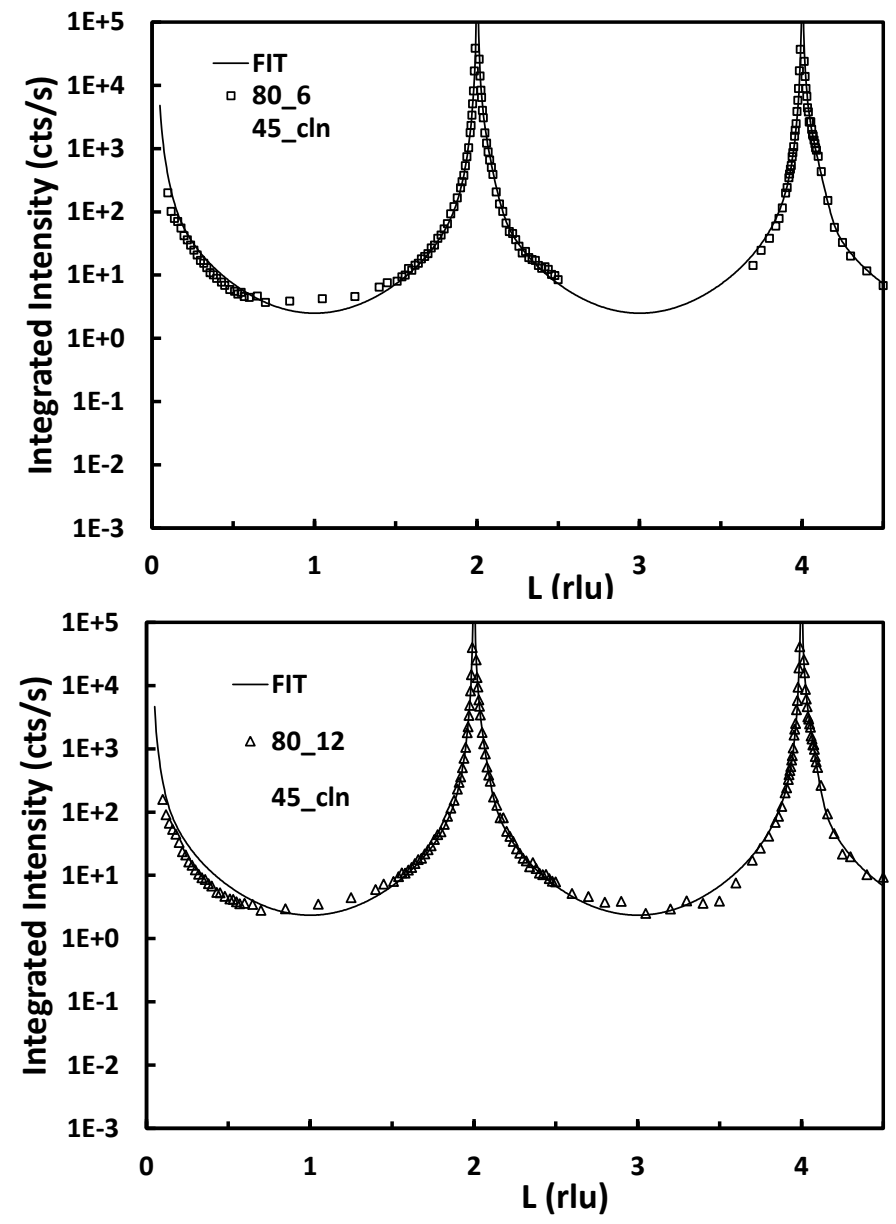

Figure 4.30: The $\Theta_{D}=80^{\circ}$ data (open symbols) for $6 \mathrm{ML}$ (top plot), 12ML (middle plot), 24ML (bottom plot) is plotted with the incoherent substrate shadowing model best fit (black solid line). This model provides a good fit to all the salient features of the three coverages: low $Q$ range, anti-Bragg, thin film oscillation spacing, and the strain near the Bragg peaks.

The incoherent shadowed substrate model best fit parameters and error bars are located in Table 4.2.

The best way to understand what is going on is to look at Figure 4.31. It is the summary of all the three different profiles: a) the top row is the density profile, b) 
the middle row is the strain profile, and c) the bottom row is the disorder profile. From left to right are the deposition angles: $0^{\circ}, 30^{\circ}, 45^{\circ}, 60^{\circ}$, and $80^{\circ}$, respectively. There are small variations in the disorder but its average value is $\alpha=2 x 10^{-3} \AA^{2}$. The density profiles all show an increasing coverage as the deposition angle increases along with an increase in the surface roughness. What is really interesting and the important part of the story is the average strain of $-1.1 \pm 0.1 \%$ that is left behind as the film grows. The profile does not change below the surface even when $\bar{N}$ is getting larger for $\Theta_{D}=80^{\circ}$. 

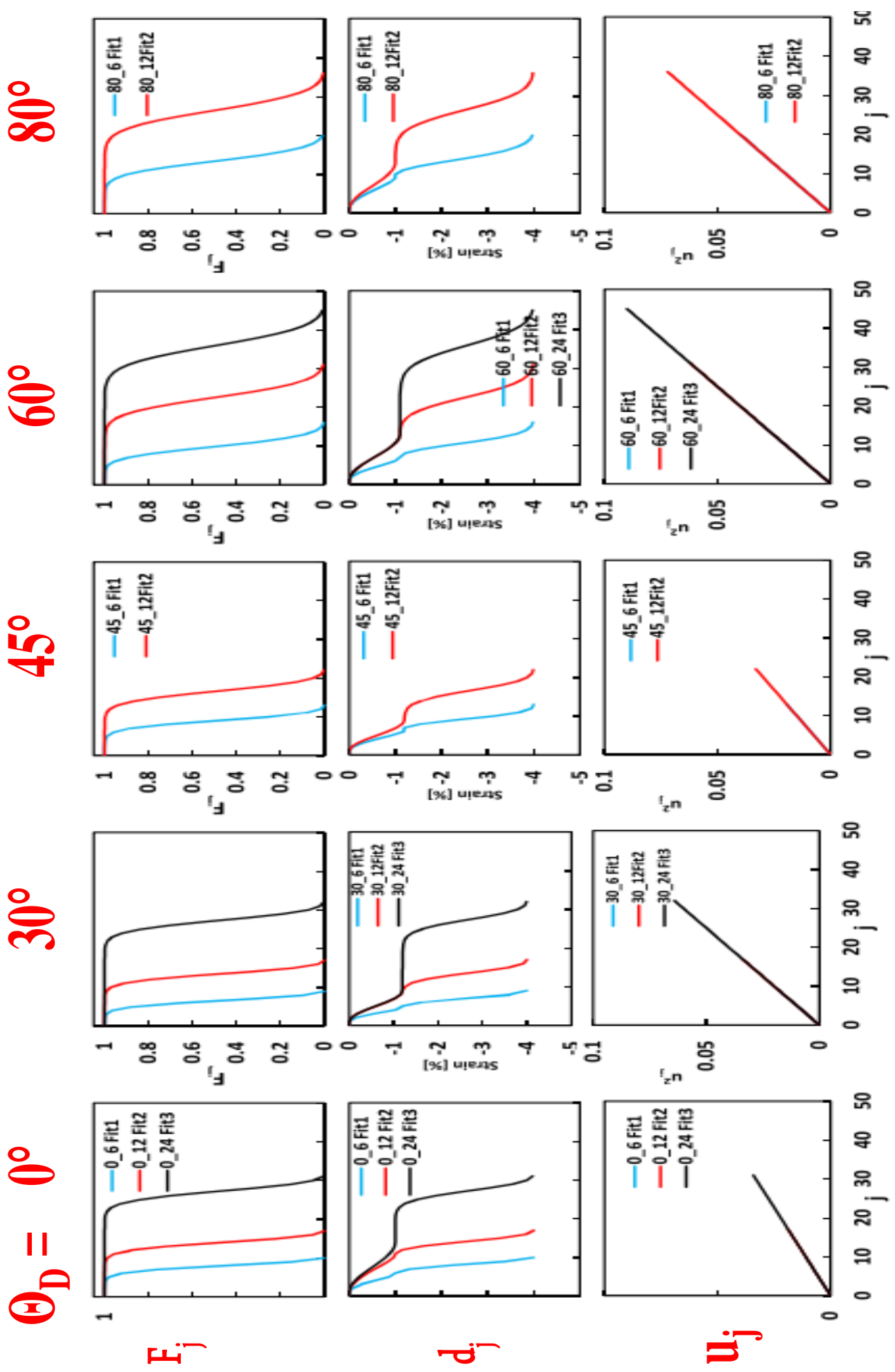

Figure 4.31: Structure, Strain, and Disorder Fitting Distributions. 
Figure 4.32 shows that the incoherent scattering coming from the substrate follows a $\tan \left(\theta_{D}\right)$, which is an experimental artifact coming from Ag mesoscopic substrate structures which shadowing during growth, and not coming from the shadowing caused by the growing film. As the deposition angle increased, the thickness of the film increased, and so did the roughness of the films, along with a slight increase in the disorder. The strain is weakly correlated with the deposition angle, but strongly correlated with the thickness, as seen in Figure 4.38.

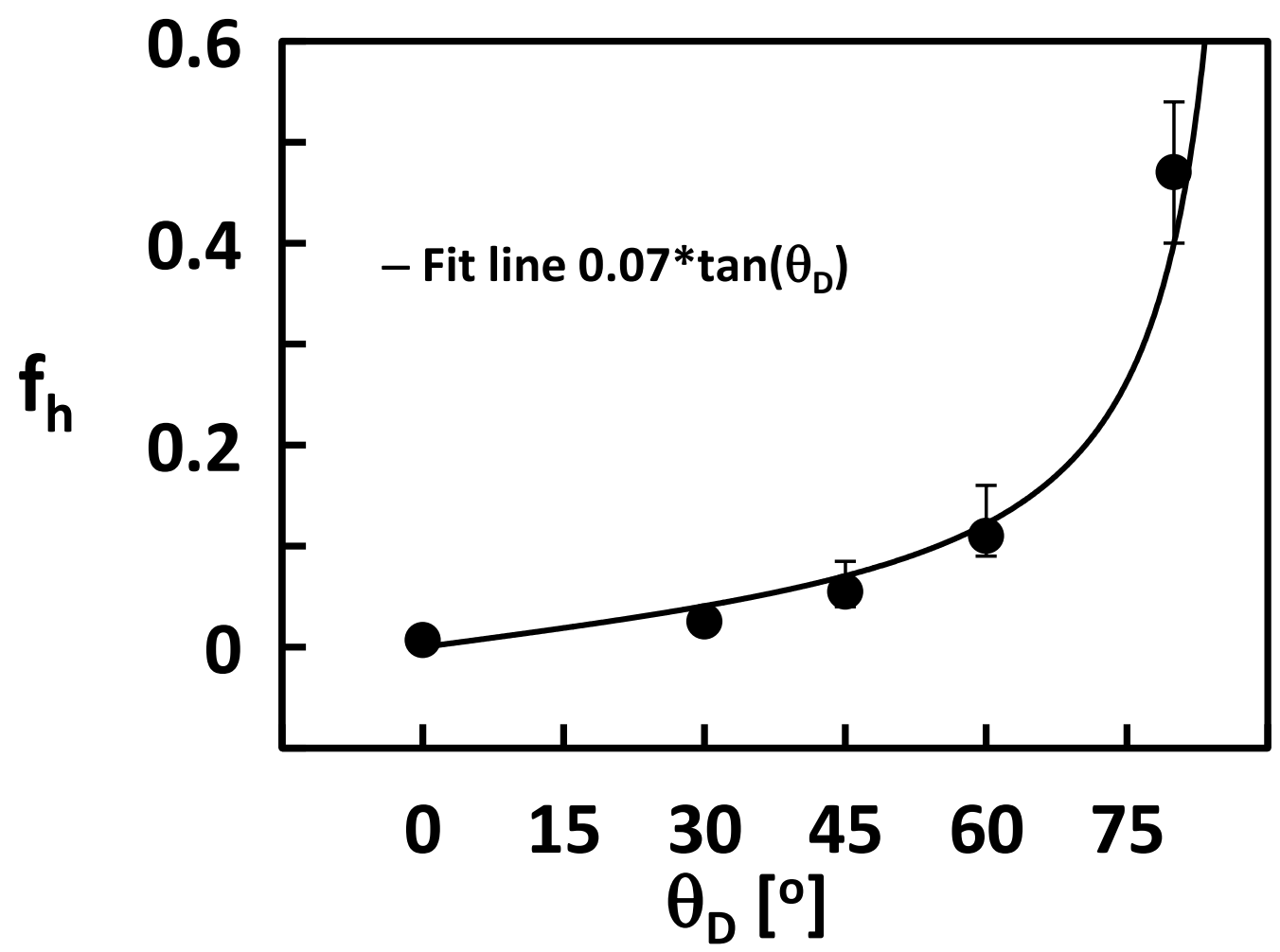

Figure 4.32: The incoherent clean surface scattering, $f_{h}$, as a function of deposition angle follows the exposed $\tan \left(\Theta_{D}\right)$ shadowing fitted line, which is able to pickup the anti-Bragg intensity data observed without inducing thin film oscillations. 


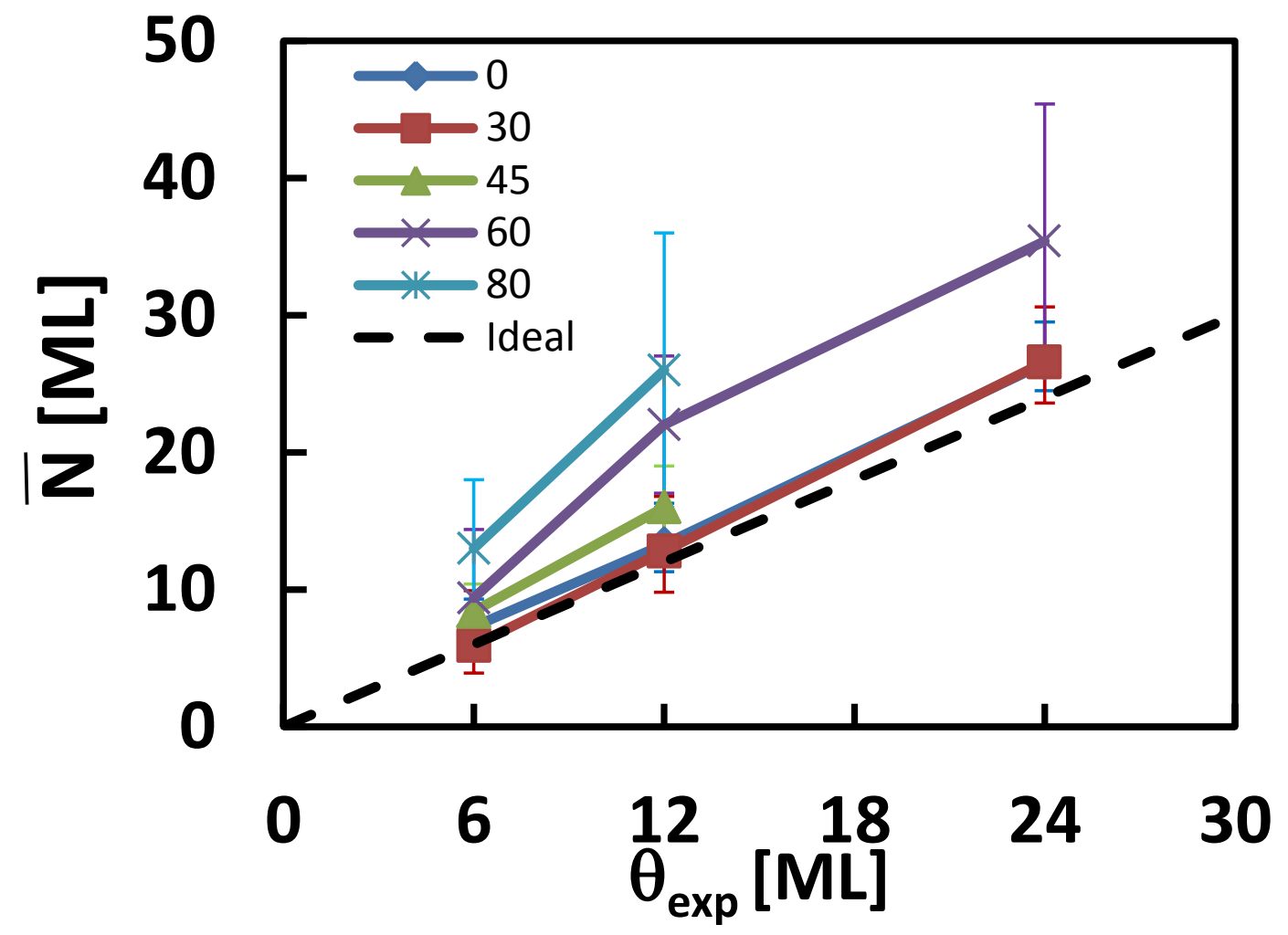

Figure 4.33: The modeled average film height, $\bar{N}$, increases as the deposition angle increases. This can be due to two effects, 1) due to the uncertainty in the deposition angle, it is possible the experimental expected coverage calibration is off, which could lead to higher or lower than expected coverage, or 2) the thickness is much larger, which would suggest a lower density film than was able to be determined from the density profile 


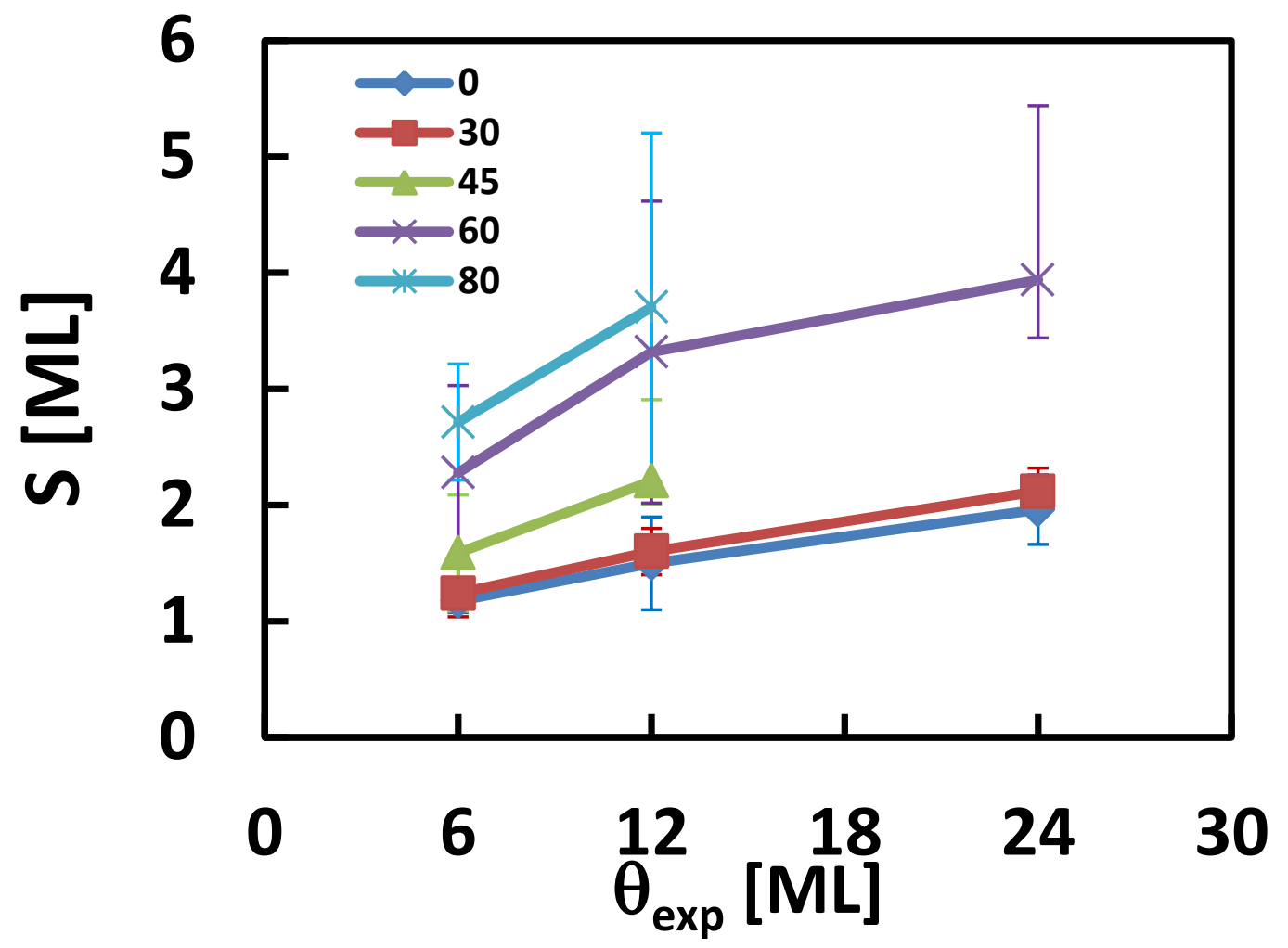

Figure 4.34: The binomial film rms roughness, $\mathrm{S}$, is plotted versus coverage as a function of deposition angle. As the deposition angle increases, there is a slight increase in the surface roughness, also an indication that the films are possibly thicker. 


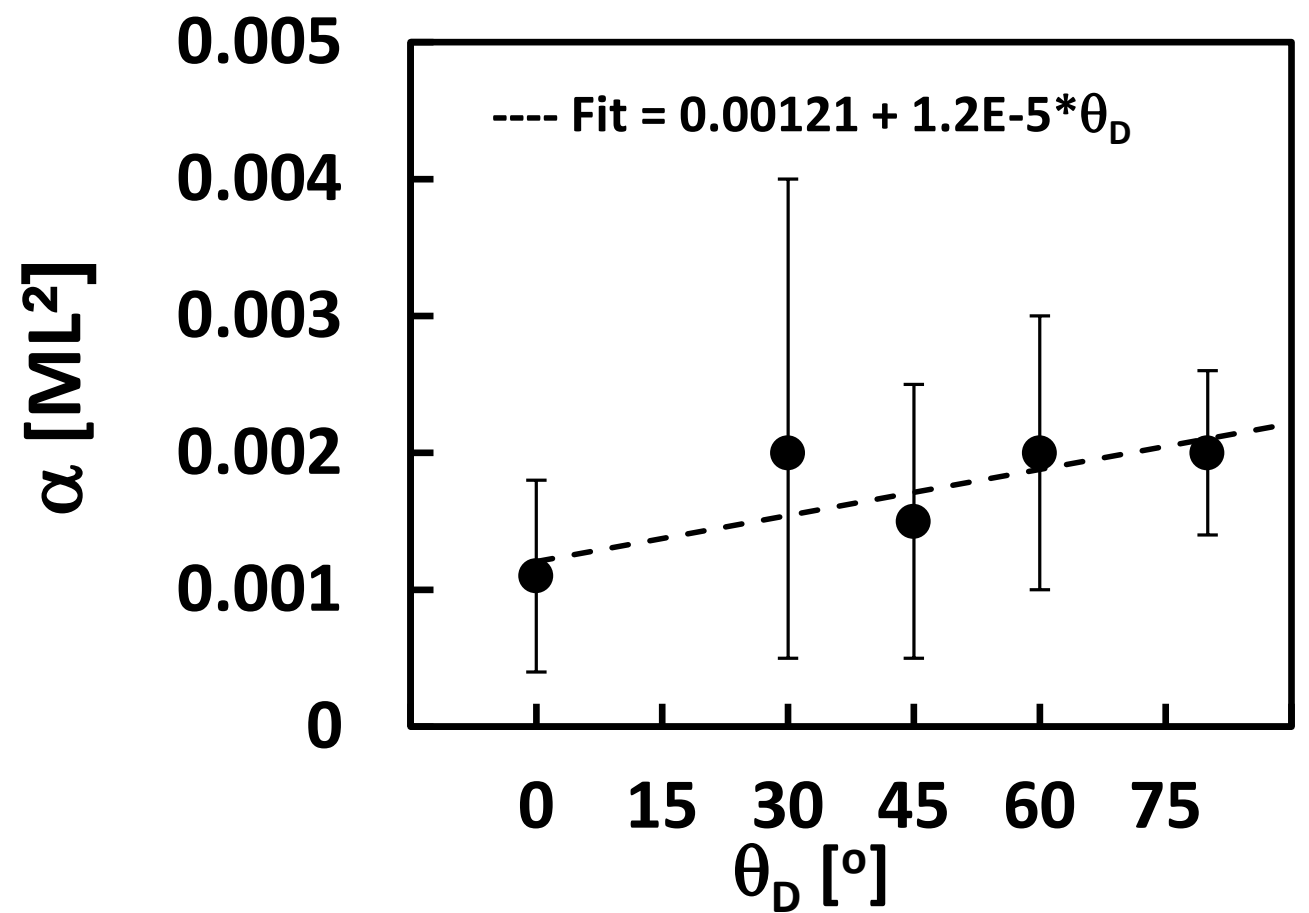

Figure 4.35: Plotted is the linear disorder parameter, $\alpha$, versus the deposition angle. The fitted line shows a slow increase in the disorder as the deposition angle increases, which correlates with the increasing coverage and increasing roughness. 


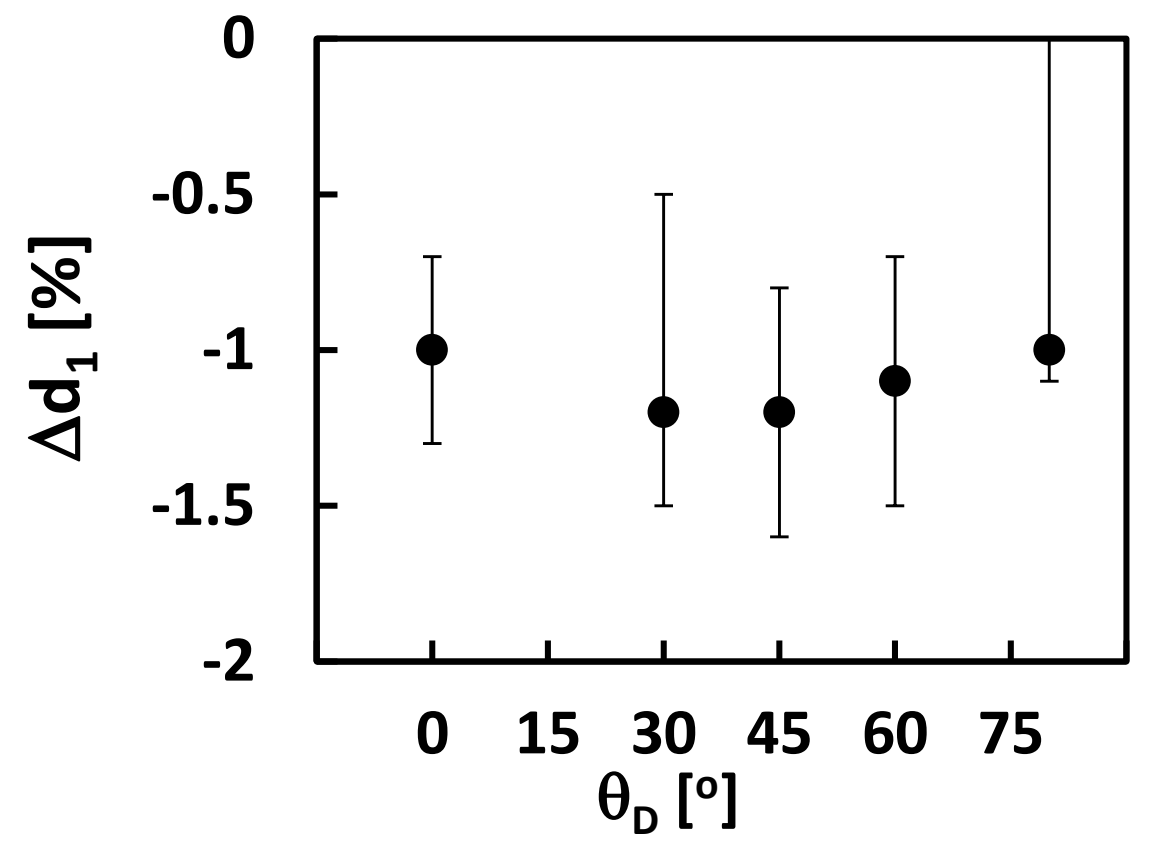

Figure 4.36: This shows $\delta d_{1}$ vs deposition angle. Much variation is not observed. 


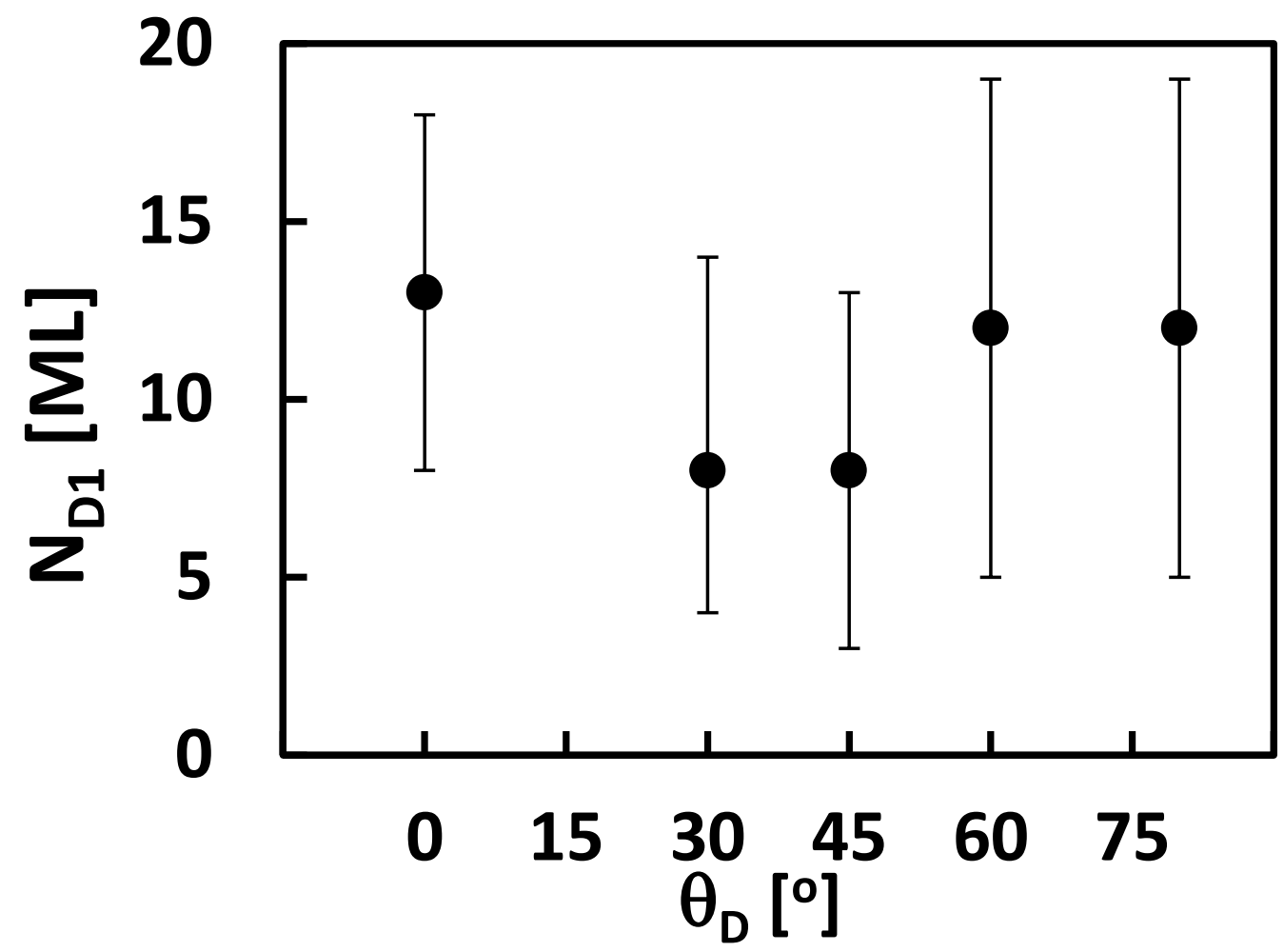

Figure 4.37: This shows $N_{D 1}$ vs deposition angle. Since is has large error bars, it could be set to 10 and try and vary $\alpha$ and $\delta d_{1}$. 


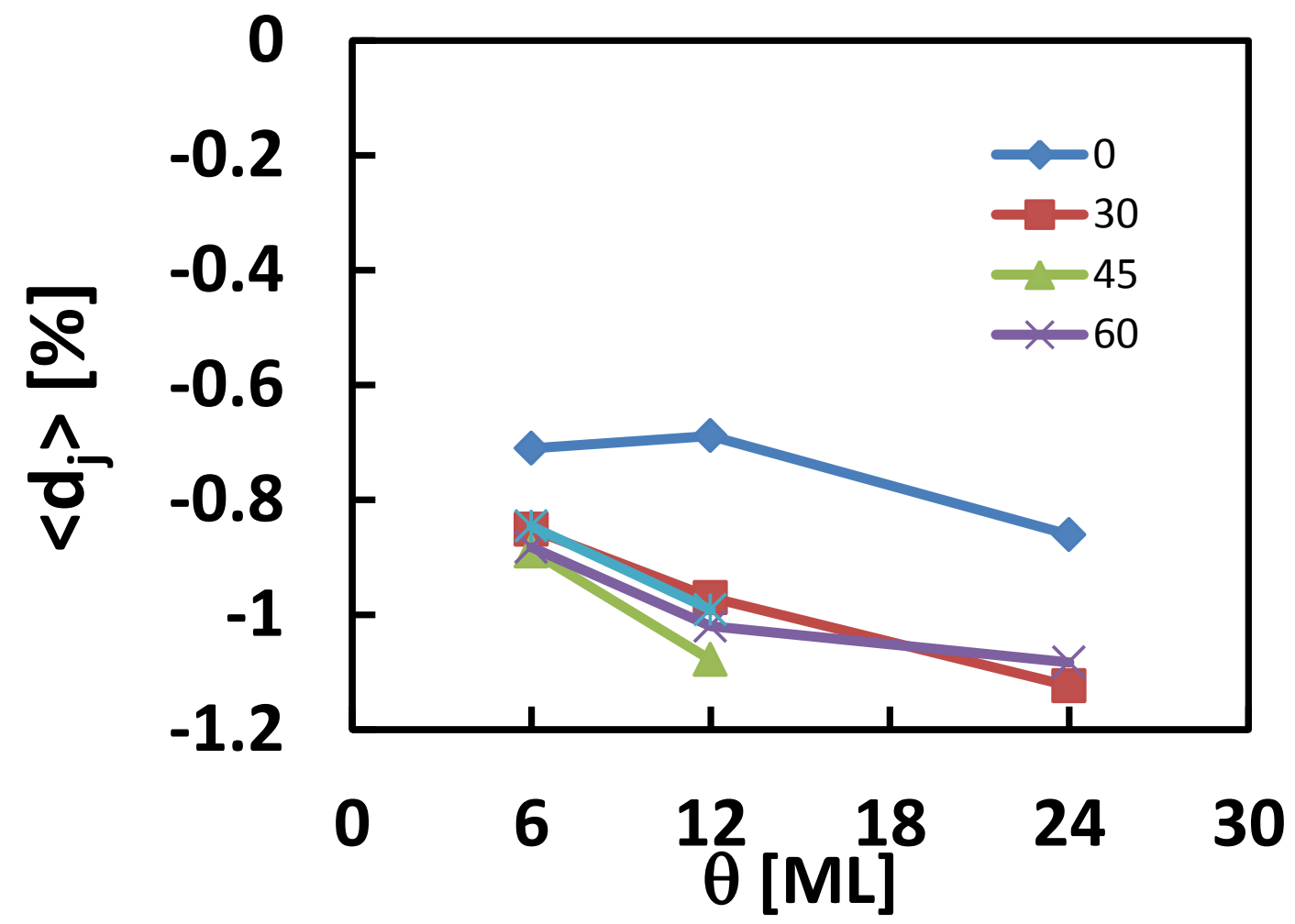

Figure 4.38: Plotted is the average strain versus coverage as a function of deposition angle. It varies as a function of coverage not as a function of deposition angle, because when the film height gets higher, the surface width roughness increases, which yields more surface strain to the average strain.

\subsubsection{Comparison: incoherent shadowed substrate model with MD simulations}

To make a comparison with MD simulations[3], a correction to the average strain for a given coverage. The author averaged over 20ML, which include the film plus the substrate. Normally, the average film strain, since it is distinguishable experimentally, is averaged over the film layers and not the substrate layers.

The following correction was made to strain plot of MD simulation [3]. The 
Table 4.2: Structure, Strain, and Disorder Fitting Parameters.

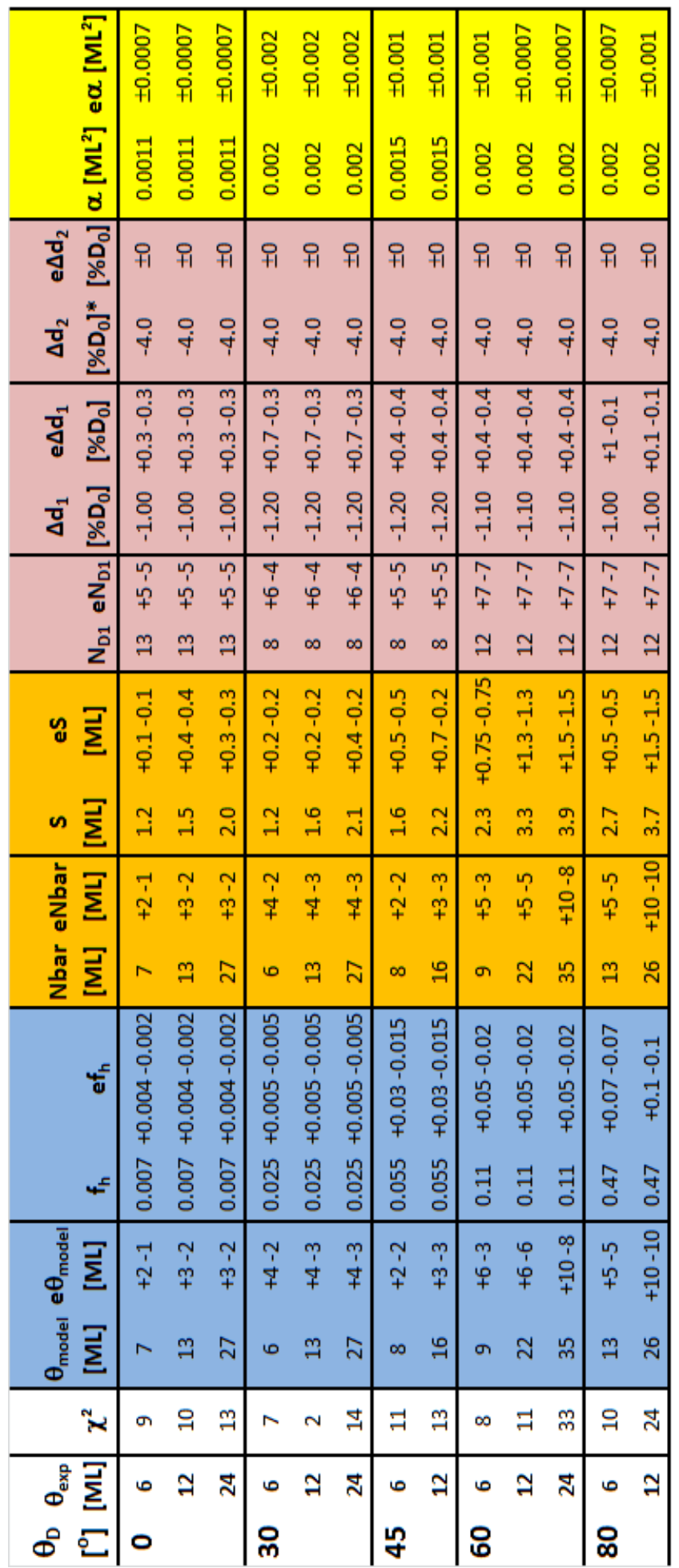


correction was computed as follows:

$$
\begin{array}{r}
<\epsilon>_{A M A R}=<\epsilon>_{20 M L}=\frac{\epsilon_{f i l m} \Theta+\epsilon_{20 M L-\Theta} \Theta}{20 M L} \\
=\frac{\epsilon \Theta}{20 M L}, \text { since } \epsilon_{20 M L-\Theta}=0 \\
\therefore \\
\epsilon_{f i l m}=\epsilon_{A M A R} \frac{20 M L}{\Theta}
\end{array}
$$

Comparing the incoherent shadowed substrate model best fit parameters from the $\mathrm{Ag} / \mathrm{Ag}(001) \mathrm{X}$-ray reflectivity to the $\mathrm{Amar}[3]$ published MD simulations for $\mathrm{Cu} / \mathrm{Cu}(001)$. The surface roughness widths are similar. As the coverage increases, the roughness increases. For low deposition angles, the average compressive film strain, as seen in Figure 4.40 are similar, but the MD calculations get enormous at $\Theta_{D}=60^{\circ}$. The experimental average strains in Figure 4.38 no show the drastic deposition angle dependence. We do not see the strains that the MD simulations predict for large deposition angles and large coverages. The $\mathrm{Ag} / \mathrm{Ag}(001)$ system is in a different growth mode than the $\mathrm{Cu} / \mathrm{Cu}(001) \mathrm{MD}$ simulated, and the point can be made that the strain buried below the surface appears to be different than what is being simulated in MD. 


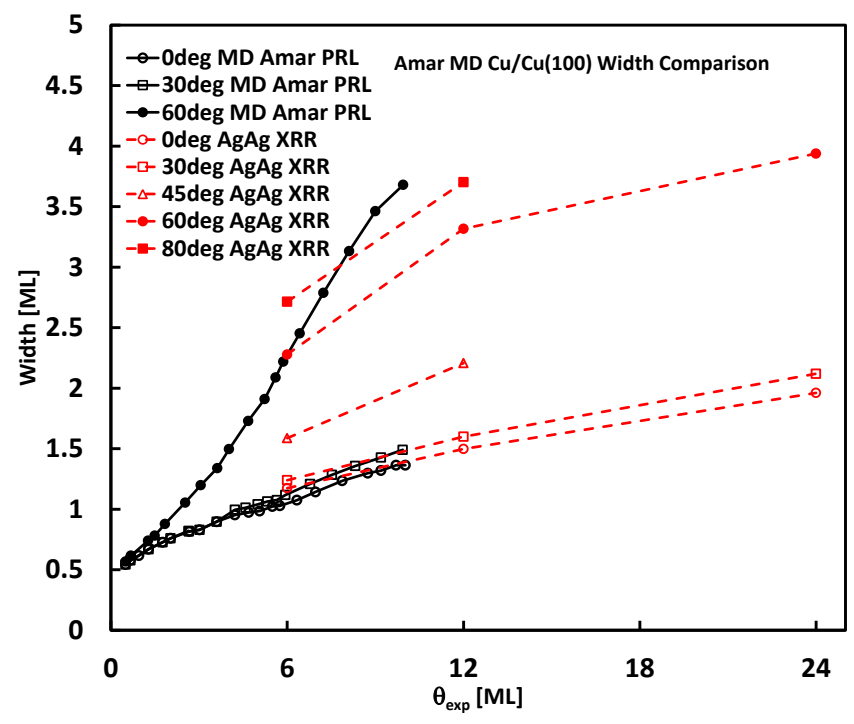

Figure 4.39: The Ag/Ag modeled surface roughness is plotted (red symbols and line) versus expected coverage as a function of deposition angle and compared to previous MD simulations calculation (black symbols and line).

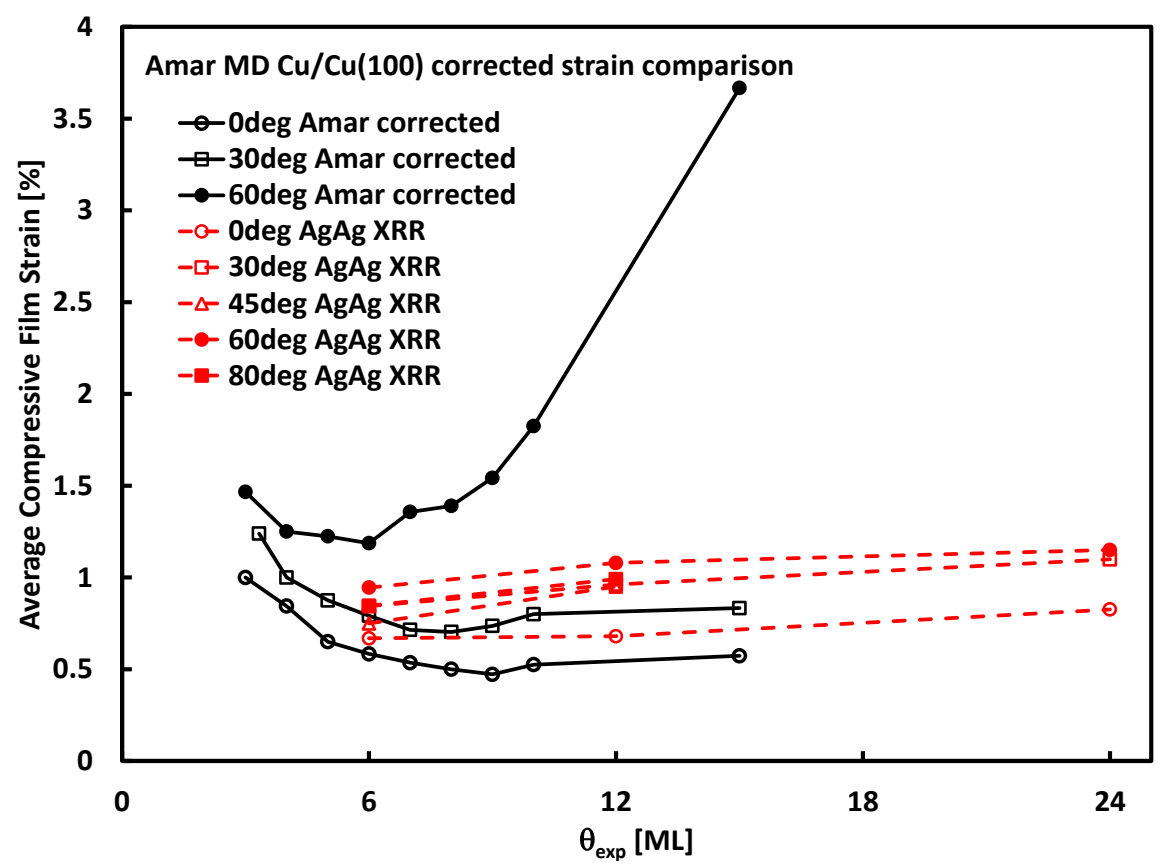

Figure 4.40: The average modeled strain is compared to previous MD simulations. 


\subsubsection{Discussion and Conclusion}

Shadowing played an important role in explaining the observed specular reflectivity for all data sets. X-ray reflectivity is not a direct measurement of vacancies buried beneath the surface. However, using deposition-angle-dependent measurements, one can reveal an evolution of average strain distribution and density distribution as the film surface morphology changes which helps to uncover the individual contributions to strain profile, the morphology and/or the vacancies.

These recent experiments cover a much larger Q space than was previously done, and that is why we now have the sensitivity to the strain distribution. Before, the reflectivity only went out to $\mathrm{L}=2$, and at the Advanced Photon Source, we are able to go out to $\mathrm{L}=4$. The further out in $\mathrm{Q}$ space you can go, the more sensitive the experiment will be to the strain.

How is it that there is always this $-1 \%$ strain in all these films, even though the surface roughness is increasing? It is hard to comprehend how an increasing morphological surface strain would lead to no noticeable changes in the strain in the bulk of the film. A straightforward explanation cannot be given. However, an easy answer is that there are vacancy concentrations left behind during film growth, that account for the strain below the surface. The $-1 \%$ strain in the bulk of the film is independent of coverage, surface roughness, and deposition angle. It is clear that there is an increase in mounding due to the increase in surface roughness, which also relate to the increase in the amount of surface strain. However, this increase in surface strain does not correlate with the $-1 \%$ strain that is left below the surface.

It appears that the surface strain is due to the exposed surface morphology having less density near the surface. It also appears that an increase in the surface roughness 
does not determine what is happening below the surface. If the strain below the surface was caused by the surface morphology strain, then I would expect the strain below the surface to change for changing surface strains, but that is not observed. It therefore leads me to believe that what is happening below the surface is independent of the exposed surface strain. Similar to how the surface strain depends on the local density or lack thereof, it would follow that strain below the exposed surface, would be induced by missing material that never filled in during film growth.

\subsection{Conclusions}

This work is focused on improving the current understanding of thin film growth by studying and modeling basic systems that show interesting growth phenomena. Of particular interest is missing material buried below the film's exposed surface. Missing material can come in the form of vacancies, vacancy clusters, overhangs, or voids that are possibly connected to the surface. There is a wealth of research in the area of epitaxial thin film growth, and yet, the lack of evidence for missing material below the exposed surface has thwarted a proper understanding of the physical growth mechanisms leading to the incorporation of missing material. Theoretical growth models intentionally remove voids, overhangs, or vacancies through mechanisms such as downward funneling[5]. This missing material can affect the physical properties of the film such as conductivity[1], and film strain[2].

An important mechanism responsible for vacancy formation has been overlooked in a simple noble metal system that is believed to be well understood. It has been shown experimentally that there are large vacancy clusters in low temperature $\mathrm{Ag} / \mathrm{Ag}(001)$ 
epitaxy[4]. Now, since missing material has been seen and measured experimentally, it can be used to provide an insight into the interplay of associated atomic-scale growth kinetics, and it should not be intentionally modeled out through the use of geometric constructs like downward funneling.

Answers to questions asked at the end of Chapter 03.

- At what deposition angle does the surface morphology strain become important and can the strain contribution from buried vacancies be determined for metal homoepitaxial growth?

- Are vacancies responsible for the observed strain in homoepitaxial systems where surface morphological structures (mounding) is suppressed?

My results show that there is sensitivity to the strain profile for deposition angles less than $60^{\circ 1}$. The strain profile does appear to have two different regions: 1) an exposed surface region that evolves and is correlated with the film thickness, rms surface roughness, and the deposition angle, and 2) a region below the exposed surface that does not appear to be correlated with the film thickness, rms surface roughness, and the deposition angle. The strained region below the surface has a near constant profile for all data sets covering 5 different deposition angles (normal to oblique) and 3 different coverages. The lack of correlation with the exposed surface would mean that the cause of the strain would have to be buried below the exposed surface. Similar to how the surface strain is proportional to the lack of nearest neighbors, it is inferred that the strain below the surface is due to vacancy clusters that were trapped and were covered up during film growth.

\footnotetext{
${ }^{1}$ The loss of sensitivity to the $60^{\circ}$ angle and above is believed to be an experimental artifact coming from the incoherent scattering due to the shadowing of mesoscopic substrate structures, and not the shadowing of the deposited film.
} 


\subsection{Acknowledgements}

Research funding is gratefully acknowledged from the National Science Foundation, DMR0706278 (MWG, STH, YC, PFM).

The Advanced Photon Source is supported by the DOE Office of Basic Energy Sciences, contract W-31-109-Eng-38. The MUCAT 6IDC beam line is supported through the Ames Lab, operated for the US DOE by Iowa State University under Contract No.W-7405-Eng-82. 


\section{Appendix A}

\section{X-ray Reflectivity Homo-Epitaxy Modeling}

This is primarily my $\mathrm{Ag} / \mathrm{Ag}$ deposition angle dependent modeling when it was believed that the anti-Bragg intensity measurements observed were dependent upon an abrupt density change starting at the buried interface.

\section{A.1 Overview}

An abrupt density change is required to obtain the anti-Bragg intensity at $\mathrm{L}=1$ and 3. This density change must occur near the $\mathrm{Ag} / \mathrm{Ag}$ interface. If the abrupt density change is near the Ag film surface, then there is too much ringing which cannot be dampened. Also, is must occur within the first 6ML of the $\mathrm{Ag} / \mathrm{Ag}$ interface in order to reduce the oscillations at high $\mathrm{Q}$ and it is seen in the experimental data, where there is no change of the intensity as a function of increasing coverage. is required to 
obtain the anti-Bragg intensity and reduce the ringing. The abrupt density change at the $\mathrm{Ag} / \mathrm{Ag}$ interface causes a large amount of ringing, and sharp cusps.

Which model to use and why. Choice of model explanation. There needs to be a jump in density in order to obtain the anti-Bragg region of the $\mathrm{X}$-ray reflectivity. However, after the abrupt density change, the transition to either and increase, decrease, or constant density cannot be determined due to a lack of sensitivity in the model predictions. Qualitatively there is no distinction in the fits. However, the missing material model yields coverage calculations much closer to the experimentally calibrated coverages. Based on the inability to qualitatively distinguish between the different density profiles, the simplest model will be used.

\section{A.2 Reflectivity Calculations for 3 layers: $\mathrm{L} 0=\mathrm{sub}$, $\mathrm{L} 1=$ transition, $\mathrm{L} 2=$ top Overview}

Goal is to calculate the reflectivity of three layers (substrate, middle, top) where the top layer roughness is nonconformal using psuedomorphic model. In the psuedomorphic model, an individual column of atoms is added discretely to obtain the scattering amplitude. Next, the column can be broken down into layers of similar atoms, and still maintain the correct amplitude. Finally, averaging over the atomic surface has to be done. This is where the rules of averaging are defined for the type of atomic distribution and assumptions about the layers being either conformal or non-conformal and the type of correlation between the amplitudes. 


\section{A.2.1 Reflectivity Calculations}

What is $\langle A\rangle$ for a conformal layer at Sub-mid, and mid-top interface and a nonconformal interface for the top layer?

Let's start.

From the Born approximation:

$$
A^{\prime}=\sum_{n=0}^{\text {all atomic layers }} \beta_{n} \exp \left(-i Q_{z} z_{n}\right)
$$

where $\beta_{n}=\frac{b_{n}}{S_{c}}=$ scattering length per area of the $n^{\text {th }}$ layer.

Assuming there are uniform layers of thickness $N$, then the amplitude can be rewritten as:

$$
A^{\prime}=\sum_{l=0}^{\text {all film layers }} A_{l} \exp \left(-i \phi_{l}\right)
$$

where $\phi_{l}=Q_{z} \sum_{j=0}^{l-1} N_{j}$, and $N_{j}=$ thickness of $j^{\text {th }}$ layer.

For a film layer,

$$
A_{l}=\beta_{l} \sum_{n=1}^{N_{l}} \exp \left(-i Q_{z} d_{l} n\right)
$$

which is a geometric series and can be written as:

$$
A_{l}=\beta_{l} \frac{\exp \left(-i Q_{z} d_{l} N_{l}\right)-1}{1-\exp \left(i Q_{z} d_{l}\right)}
$$

Now, for a discrete case of 3 layers, where $\mathrm{L} 0=$ substrate, $\mathrm{L} 1=$ middle layer, and L2= top layer, each layers amplitude follows equation A.3, except for the substrate. 
The substrate is semi-infinite:

$$
A_{0}=\beta_{0} \sum_{n=-\infty}^{N_{0}} \exp \left(-i Q_{z} d_{0} n\right),
$$

which becomes:

$$
A_{0}=\beta_{0} \frac{\exp \left(-i Q_{z} d_{0} N_{0}\right)}{1-\exp \left(i Q_{z} d_{0}\right)} .
$$

The other layers are:

$$
\begin{aligned}
& A_{1}=\beta_{1} \frac{\exp \left(-i Q_{z} d_{1} N_{1}\right)-1}{1-\exp \left(i Q_{z} d_{1}\right)}, \\
& A_{2}=\beta_{2} \frac{\exp \left(-i Q_{z} d_{2} N_{2}\right)-1}{1-\exp \left(i Q_{z} d_{2}\right)} .
\end{aligned}
$$

The total amplitude is:

$$
\begin{gathered}
A^{\prime}=\sum_{l=0}^{3} A_{l} \exp \left(-i \phi_{l}\right), \\
A^{\prime}=A_{0} \\
+A_{1} \exp \left(-i Q_{z} \Delta_{01}\right) \exp \left(-i Q_{z} d_{0} N_{0}\right) \\
+A_{2} \exp \left(-i Q_{z} \Delta_{01}\right) \exp \left(-i Q_{z} d_{0} N_{0}\right) \exp \left(-i Q_{z} \Delta_{12}\right) \exp \left(-i Q_{z} d_{1} N_{1}\right),
\end{gathered}
$$

where $\Delta_{i-1, i}$ is the discrete offset of the film layer $i$, which allows a different spacing at the interface than the film lattice spacing $d_{i}$.

\section{A.2.2 Averaging}

Now, the amplitude needs to be averaged over the surface. Assumptions: 1) Layer 1 is conformal to Layer0, N0 is uncorrelated, 2) Layer2 is nonconformal to Layer1, but 
conformal to Layer0, N1 and N2 are correlated (non-conformal top layer).

For the amplitude, the average of the sum is the sum of the average:

$$
\begin{aligned}
\left\langle A^{\prime}\right\rangle & =\left\langle A_{0}\right\rangle \\
& +\left\langle A_{1} \exp \left(-i Q_{z} \Delta_{01}\right) \exp \left(-i Q_{z} d_{0} N_{0}\right)\right\rangle \\
& +\left\langle A_{2} \exp \left(-i Q_{z} \Delta_{01}\right) \exp \left(-i Q_{z} d_{0} N_{0}\right) \exp \left(-i Q_{z} \Delta_{12}\right) \exp \left(-i Q_{z} d_{1} N_{1}\right)\right\rangle .
\end{aligned}
$$

For the first term in equation A.7:

$$
\begin{aligned}
\left\langle A_{0}\right\rangle & =\left\langle\beta_{0} \frac{\exp \left(-i Q_{z} d_{0} N_{0}\right)}{1-\exp \left(i Q_{z} d_{0}\right)}\right\rangle \\
& =\beta_{0} \frac{\left\langle\exp \left(-i Q_{z} d_{0} N_{0}\right)\right\rangle}{\left\langle 1-\exp \left(i Q_{z} d_{0}\right)\right\rangle} \\
& =\beta_{0} V_{0} \frac{1}{1-\exp \left(i Q_{z} d_{0}\right)},
\end{aligned}
$$

where $V_{0}=\left\langle\exp \left(-i Q_{z} d_{0} N_{0}\right)\right\rangle_{N_{0}}$.

For the second term in equation A.7:

$$
\begin{aligned}
& \left\langle A_{1} \exp \left(-i Q_{z} \Delta_{01}\right) \exp \left(-i Q_{z} d_{0} N_{0}\right)\right\rangle \\
& =\left\langle\beta_{1} \frac{\exp \left(-i Q_{z} d_{1} N_{1}\right)-1}{1-\exp \left(i Q_{z} d_{1}\right)} \exp \left(-i Q_{z} \Delta_{01}\right) \exp \left(-i Q_{z} d_{0} N_{0}\right)\right\rangle,
\end{aligned}
$$

since $N_{0}$ and $N_{1}$ are uncorrelated, the average of the products is the product of the averages

$$
\begin{aligned}
& =\beta_{1} \frac{\left\langle\exp \left(-i Q_{z} d_{1} N_{1}\right)-1\right\rangle}{\left\langle 1-\exp \left(i Q_{z} d_{1}\right)\right\rangle}\left\langle\exp \left(-i Q_{z} \Delta_{01}\right)\right\rangle\left\langle\exp \left(-i Q_{z} d_{0} N_{0}\right)\right\rangle \\
& =\beta_{1} V_{0} \nu_{01} \frac{V_{1}-1}{1-\exp \left(i Q_{z} d_{1}\right)},
\end{aligned}
$$

where $V_{1}=\left\langle\exp \left(-i Q_{z} d_{1} N_{1}\right)\right\rangle_{N_{1}}$ and $\nu_{01}=\left\langle\exp \left(-i Q_{z} \Delta_{01}\right)\right\rangle$. 
For the third term in equation A.7:

$$
\begin{aligned}
& \left\langle A_{2} \exp \left(-i Q_{z} \Delta_{01}\right) \exp \left(-i Q_{z} d_{0} N_{0}\right) \exp \left(-i Q_{z} \Delta_{12}\right) \exp \left(-i Q_{z} d_{1} N_{1}\right)\right\rangle \\
& =\left\langle\beta_{2} \frac{\exp \left(-i Q_{z} d_{2} N_{2}\right)-1}{1-\exp \left(i Q_{z} d_{2}\right)} \exp \left(-i Q_{z} \Delta_{01}\right) \exp \left(-i Q_{z} d_{0} N_{0}\right) \exp \left(-i Q_{z} \Delta_{12}\right) \exp \left(-i Q_{z} d_{1} N_{1}\right)\right\rangle,
\end{aligned}
$$

since $N_{1}$ and $N_{2}$ are correlated, the averages have to be done carefully

$$
\begin{aligned}
& =\beta_{2}\left\langle\frac{\exp \left(-i Q_{z} d_{2} N_{2}\right)-1}{\left\langle 1-\exp \left(i Q_{z} d_{2}\right)\right\rangle} \exp \left(-i Q_{z} d_{1} N_{1}\right)\right\rangle\left\langle\exp \left(-i Q_{z} \Delta_{01}\right)\right\rangle\left\langle\exp \left(-i Q_{z} d_{0} N_{0}\right)\right\rangle\left\langle\exp \left(-i Q_{z} \Delta_{12}\right)\right\rangle \\
& =\beta_{2} V_{0} \nu_{01} \nu_{12} \frac{\left\langle\exp \left(-i Q_{z}\left(d_{2} N_{2}+d_{1} N_{2}\right)\right)-\exp \left(-i Q_{z} d_{1} N_{1}\right)\right\rangle}{\left\langle 1-\exp \left(i Q_{z} d_{1}\right)\right\rangle},
\end{aligned}
$$

numerator and denominator are uncorrelated

$$
\begin{aligned}
& =\beta_{2} V_{0} \nu_{01} \nu_{12} \frac{\left\langle\exp \left(-i Q_{z}\left(d_{2} N_{2}+d_{1} N_{2}\right)\right)\right\rangle-\left\langle\exp \left(-i Q_{z} d_{1} N_{1}\right)\right\rangle}{\left\langle 1-\exp \left(i Q_{z} d_{2}\right)\right\rangle}, \\
& =\beta_{2} V_{0} \nu_{01} \nu_{12} \frac{V_{2}^{\prime}-V_{1}}{1-\exp \left(i Q_{z} d_{2}\right)}, \\
& =\beta_{2} V_{0} V_{1} \nu_{01} \nu_{12} \frac{\frac{V_{2}^{\prime}}{V_{1}}-1}{1-\exp \left(i Q_{z} d_{2}\right)}, \\
& =\beta_{2} V_{0} V_{1} \nu_{01} \nu_{12} \frac{V_{2}^{\prime \prime}-1}{1-\exp \left(i Q_{z} d_{2}\right)},
\end{aligned}
$$

where $V_{2}^{\prime \prime}=\frac{V_{2}^{\prime}}{V_{1}}, V_{2}^{\prime}=\left\langle\exp \left(-i Q_{z}\left(d_{2} N_{2}+d_{1} N_{1}\right)\right)\right\rangle$ and $\nu_{12}=\left\langle\exp \left(-i Q_{z} \Delta_{12}\right)\right\rangle$.

Rewriting the average amplitude:

$$
\begin{aligned}
\left\langle A^{\prime}\right\rangle & =\beta_{0} V_{0} \frac{1}{1-\exp \left(i Q_{z} d_{0}\right)} \\
& +\beta_{1} V_{0} \nu_{01} \frac{V_{1}-1}{1-\exp \left(i Q_{z} d_{1}\right)}, \\
& +\beta_{2} V_{0} V_{1} \nu_{01} \nu_{12} \frac{V_{2}^{\prime \prime}-1}{1-\exp \left(i Q_{z} d_{2}\right)} .
\end{aligned}
$$




\section{A.2.3 $V_{2}^{\prime \prime}$}

The roughness of Layer 2 looks different than $V_{0}$ and $V_{1}$.

$$
V_{2}^{\prime \prime}=\frac{V_{2}^{\prime}}{V_{1}},
$$

where,

$$
V_{2}^{\prime}=\left\langle\exp \left(-i Q_{z}\left(d_{2} N_{2}+d_{1} N_{1}\right)\right)\right\rangle .
$$

CASE 1: If we assume $d_{2}=d_{1}$, then

$$
\begin{aligned}
V_{2}^{\prime} & =\left\langle\exp \left(-i Q_{z}\left(d_{2} N_{2}+d_{1} N_{1}\right)\right)\right\rangle \\
& =\left\langle\exp \left(-i Q_{z} d_{1}\left(N_{2}+N_{1}\right)\right)\right\rangle \\
& =\left\langle\exp \left(-i Q_{z} d_{1} N^{\prime}\right)\right\rangle \\
& =\left\langle\exp \left(-i Q_{z} d_{1} N^{\prime}\right)\right\rangle
\end{aligned}
$$

where $N^{\prime}=N_{1}+N_{2}$.

CASE 2: If $d_{2}=d_{1}+\Delta d_{12}$, then

$$
\begin{aligned}
V_{2}^{\prime} & =\left\langle\exp \left(-i Q_{z}\left(d_{2} N_{2}+d_{1} N_{1}\right)\right)\right\rangle \\
& =\left\langle\exp \left(-i Q_{z}\left(\left(d_{1}+\Delta d_{12}\right) N_{2}+d_{1} N_{1}\right)\right)\right\rangle \\
& =\left\langle\exp \left(-i Q_{z} d_{1}\left(N_{2}+N_{1}\right)\right) \exp \left(-i Q_{z} \Delta d_{12} N_{2}\right)\right\rangle \\
& =\left\langle\exp \left(-i Q_{z} d_{1} N^{\prime}\right) \exp \left(-i Q_{z} \Delta d_{12} N_{2}\right)\right\rangle
\end{aligned}
$$

where $N^{\prime}=N_{1}+N_{2}$.

We know how to do the averaging for $\exp \left(-i Q_{z} d_{1} N^{\prime}\right)$, but I am not sure how to calculate $V_{2}^{\prime}$. Make assumption that $d_{12} N_{2}$ is uncorrelated to $d_{1} N^{\prime}$ since $d_{12}$ is very small. 
Then,

$$
\begin{aligned}
V_{2}^{\prime} & =\left\langle\exp \left(-i Q_{z} d_{1} N^{\prime}\right) \exp \left(-i Q_{z} \Delta d_{12} N_{2}\right)\right\rangle \\
& =\left\langle\exp \left(-i Q_{z} d_{1} N^{\prime}\right)\right\rangle\left\langle\exp \left(-i Q_{z} \Delta d_{12} N_{2}\right)\right\rangle \\
& =V_{2}^{\prime \prime \prime} \eta_{12}
\end{aligned}
$$

\section{A.2.4 Conclusion}

The average amplitude has the same form as conformal roughness, but the difference is the averaging of the top layer $V_{2}^{\prime \prime}$. $V_{2}^{\prime \prime}$ reduces to $V_{2}$ for conformal roughness.

The average amplitude from equation A.11 is:

$$
\begin{aligned}
\left\langle A^{\prime}\right\rangle & =\beta_{0} V_{0} \frac{1}{1-\exp \left(i Q_{z} d_{0}\right)} \\
& +\beta_{1} V_{0} \nu_{01} \frac{V_{1}-1}{1-\exp \left(i Q_{z} d_{1}\right)} \\
& +\beta_{2} V_{0} V_{1} \nu_{01} \nu_{12} \frac{V_{2}^{\prime \prime}-1}{1-\exp \left(i Q_{z} d_{2}\right)}
\end{aligned}
$$

where $\beta_{i}=\frac{b_{n}}{S_{c}}$ (scattering length density per unit area), $V_{i}=\left\langle\left(\exp \left(-i Q_{z} d_{i} N_{i}\right)\right)\right\rangle$, $d_{i}$ is the $i^{t h}$ layer d-spacing, $\nu_{i-1, i}=\exp \left(-i Q_{z} \Delta_{i-1, i}\right), \Delta_{i-1, i}$ is the additional layer spacing between layers $i-1$ and $i, V_{2}^{\prime \prime}=\frac{V_{2}^{\prime}}{V 1}$, $V_{2}^{\prime}=\left\langle\exp \left(-i Q_{z} d_{1} N^{\prime}\right)\right\rangle\left\langle\exp \left(-i Q_{z} \Delta d_{12} N_{2}\right)\right\rangle$ (eqn A.14), and $\Delta d_{12}=d_{2}-d_{1}$.

For computational reasons $\left(V_{2}^{\prime}-V_{1}\right.$ is much simpler than $\left.V_{1} *\left(\frac{V_{2}^{\prime}}{V_{1}}-1\right)\right)$, the average 
amplitude from equation A.11 will be programmed for Case 1: $d_{1}=d_{2}$ as follows:

$$
\begin{aligned}
\left\langle A^{\prime}\right\rangle & =\beta_{0} V_{0} \frac{1}{1-\exp \left(i Q_{z} d_{0}\right)} \\
& +\beta_{1} V_{0} \nu_{01} \frac{V_{1}-1}{1-\exp \left(i Q_{z} d_{1}\right)}, \\
& +\beta_{2} V_{0} \nu_{01} \nu_{12} \frac{V_{2}^{\prime}-V_{1}}{1-\exp \left(i Q_{z} d_{2}\right)}
\end{aligned}
$$

Averaging using the binomial distribution is known, so it will not shown here.

\section{A.3 Abrupt Transistion Fits}

Below are the fits for the $\Theta_{D}=0^{\circ}$ specular X-ray reflectivity using the krefl3_nc_reswin.5.c code. So far, I have been able for the $0^{\circ}$ data to keep L1 the same for all data sets: 6,12 , and 24ML. However, the data fit for 24ML doesn't look any better than for a single layer. On the other hand, I do pick seem to pick up the lower Q bragg peak intensity and oscillation better, and I also get the coverage correct.

\section{A.3.1 Conclusion}

This model will not work. The abrupt density transition caused too much ringing. It picks up the anti-Bragg at the expense of picking up other salient features of the data.

A model needs to be choosen such that it can obtain the anti-Bragg intensity and at the same time not introduce excessive ringing. 


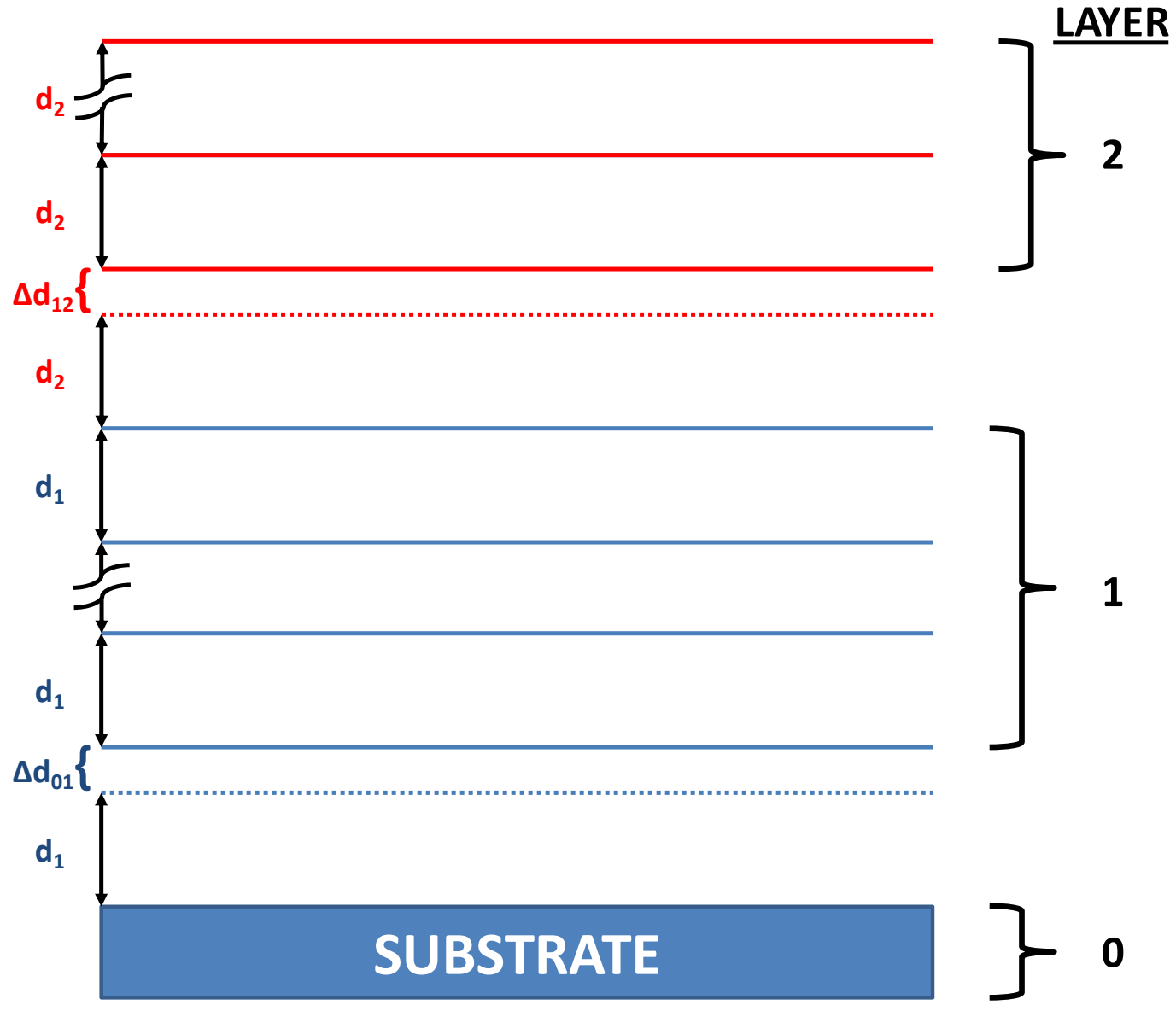

Figure A.1: Schematic showing the 3-Layer Model used to fit the following data sets. 

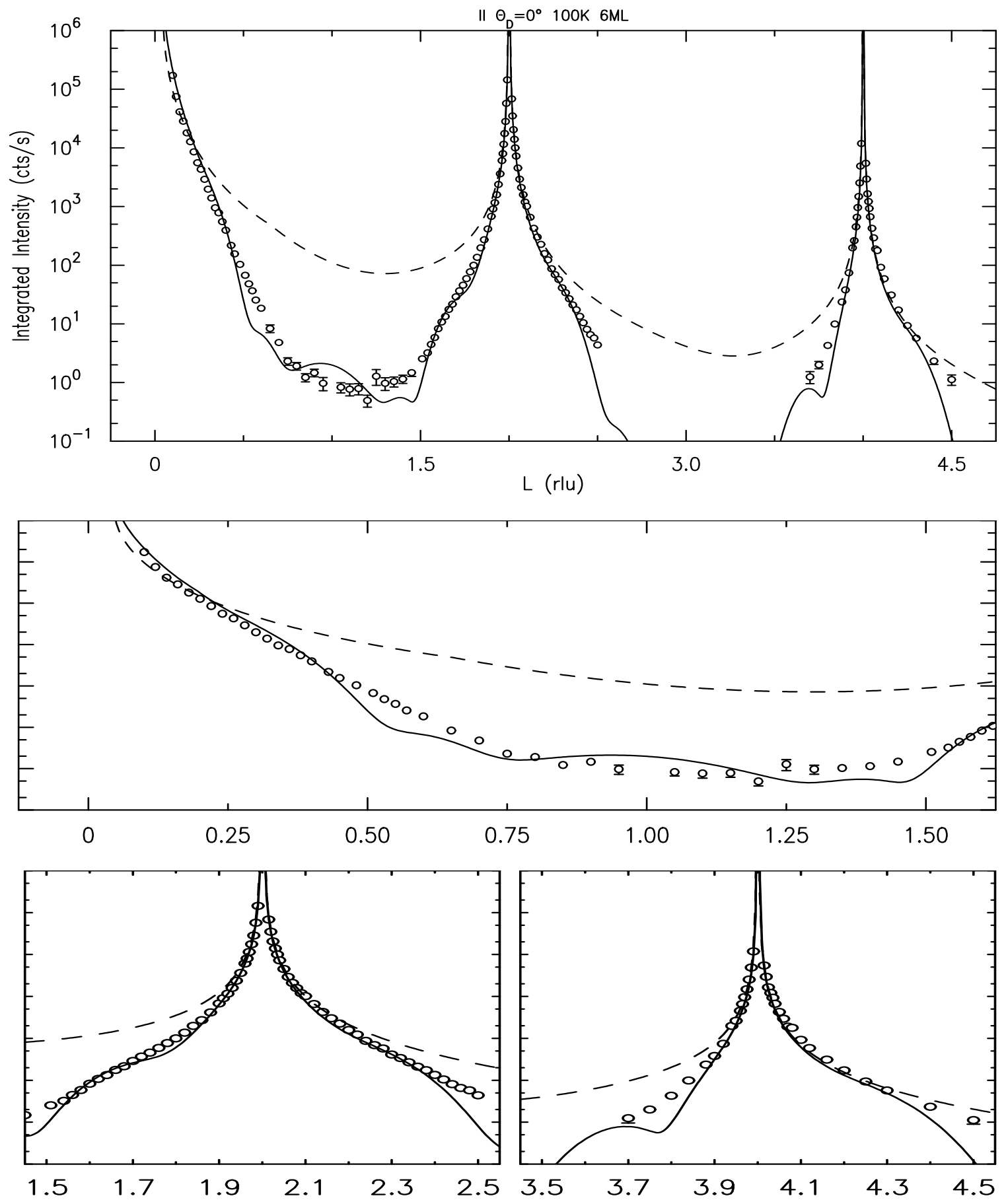

Figure A.2: Top plot is $\Theta_{D}=0^{\circ} 6 \mathrm{ML}$ reflectivity data (open circles) and the 3 Layer Model fit (solid line). The other panels are zoomed in regions. 

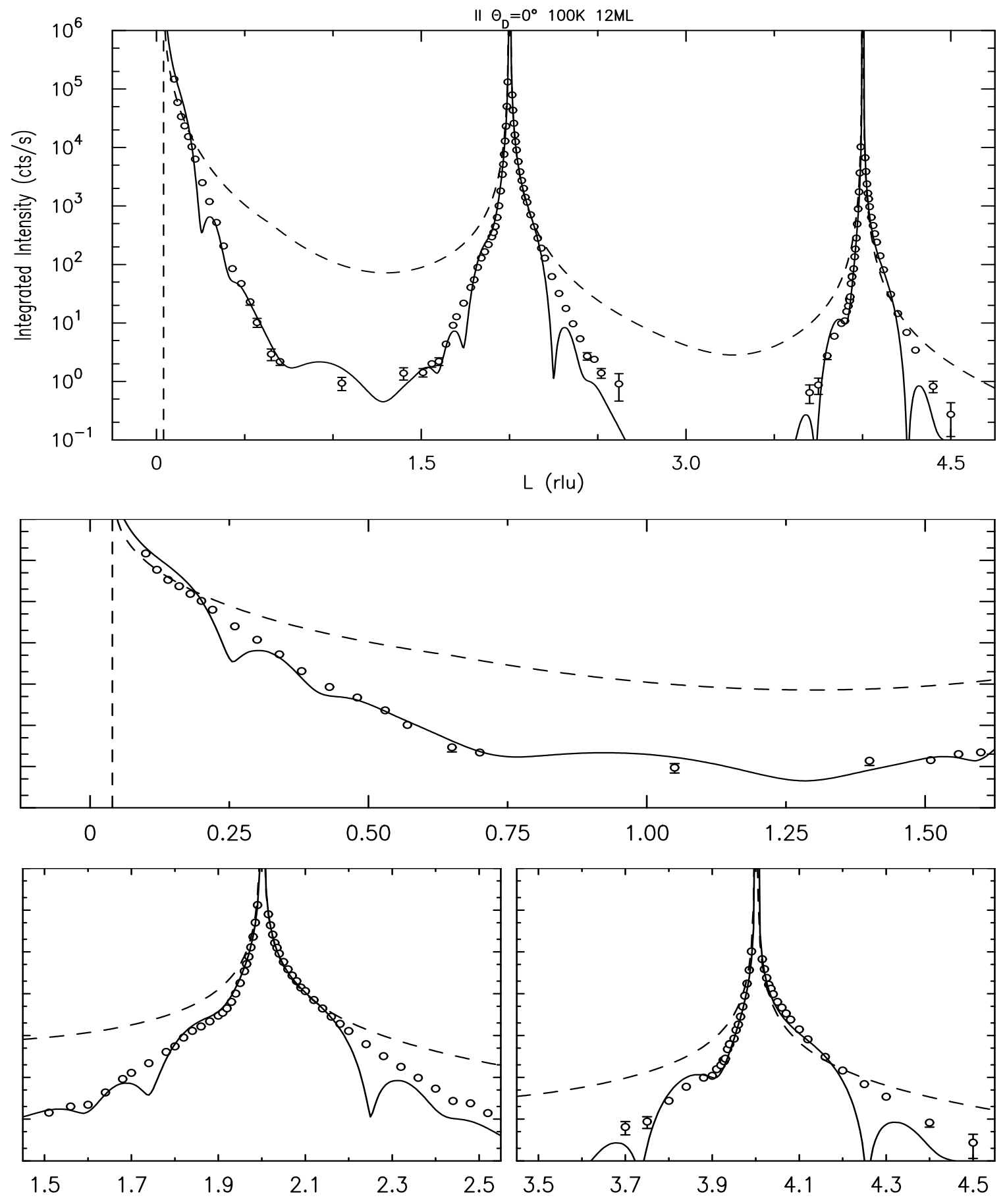

Figure A.3: Top plot is $\Theta_{D}=0^{\circ} 12 \mathrm{ML}$ reflectivity data (open circles) and the 3 Layer Model fit (solid line). The other panels are zoomed in regions. 

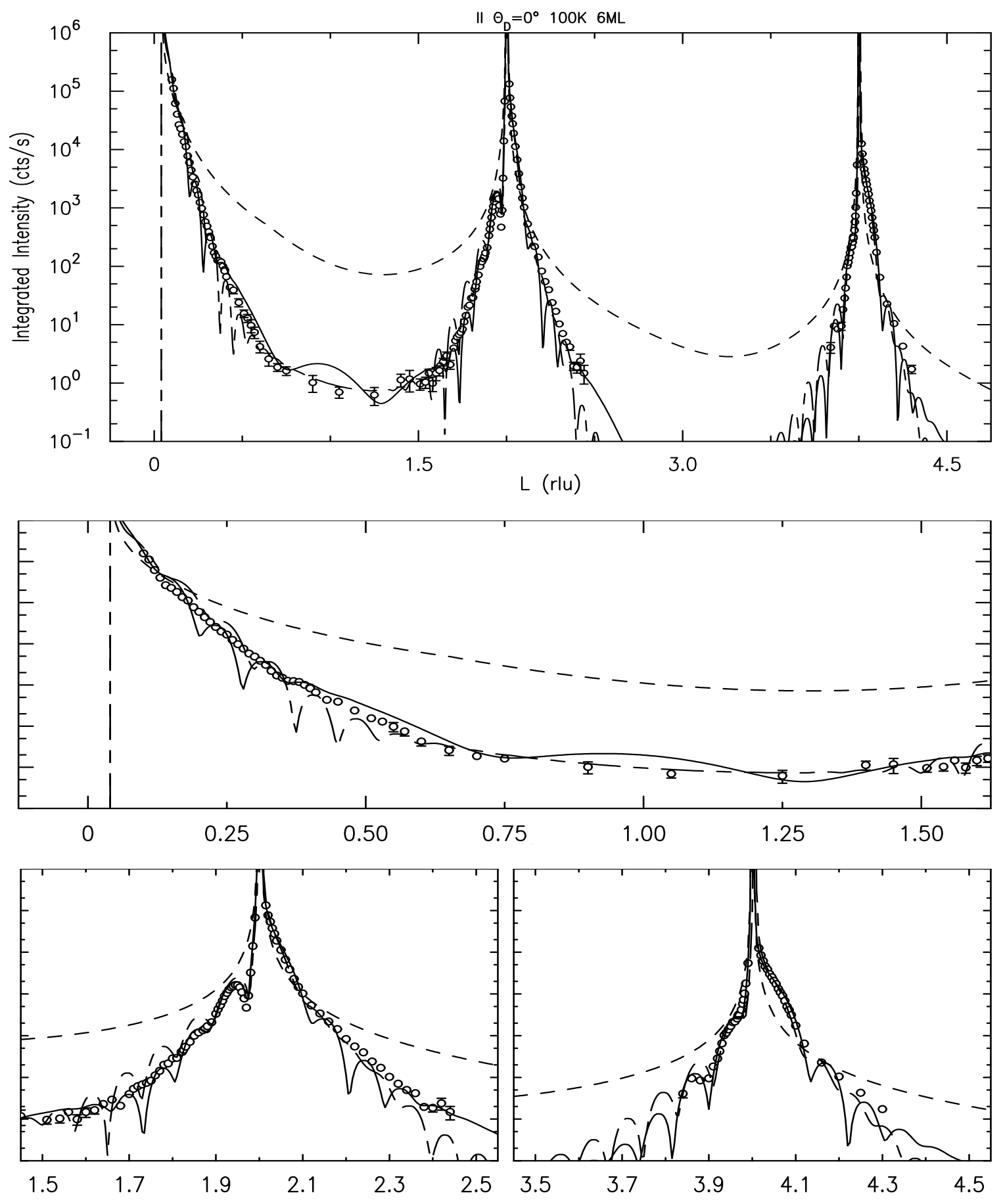

Figure A.4: Top plot is $\Theta_{D}=0^{\circ} 12 \mathrm{ML}$ reflectivity data (open circles), the 3-Layer Model fit (solid line) and 2-Layer model (long dash short dash). Neither fit looks better than the other, however, the 3-layer model picks up the lower Q bragg peak intensity and oscillation better, while ringing too much on the high $\mathrm{Q}$ side of the bragg peak, and the 3-layer model also yields the expected coverage to less than $4 \%$. The other panels are zoomed in regions. 100 


\section{Bibliography}

[1] S. B. Arnason, S. P. Herschfield, and A. F. Hebard. Bad metals made with good-metal components. Physical Review Letters, 81(18):3936-3939, 1998.

[2] C. E. Botez, K. Li, E. D. Lu, W. C. Elliott, P. F. Miceli, E. H. Conrad, and P. W. Stephens. Vacancy trapping and annealing in noble-metal films grown at low temperature. Applied Physics Letters, 81(25):4718-4720, 2002.

[3] Yunsic Shim, Valery Borovikov, Blas P. Uberuaga, Arthur F. Voter, and Jacques G. Amar. Vacancy formation and strain in low-temperature cu/cu(100) growth. Physical Review Letters, 101(11):116101, 2008.

[4] Chinkyo Kim, Rui Feng, Edward H. Conrad, and Paul F. Miceli. Nanoclustering of vacancies in thin metal films revealed by x-ray diffuse scattering. Applied Physics Letters, 91(9):093131, 2007.

[5] J. W. Evans, D. E. Sanders, P. A. Thiel, and Andrew E. DePristo. Lowtemperature epitaxial growth of thin metal films. Physical Review B, 41(8):54105413, 1990. PRB. 
[6] J. W. Evans, P. A. Thiel, and M. C. Bartelt. Morphological evolution during epitaxial thin film growth: Formation of 2d islands and 3d mounds. Surface Science Reports, 61(1-2):1-128, 2006. doi: DOI: 10.1016/j.surfrep.2005.08.004.

[7] Gert Ehrlich and F. G. Hudda. Atomic view of surface self-diffusion: Tungsten on tungsten. The Journal of Chemical Physics, 44(3):1039-1049, 1966.

[8] Richard L. Schwoebel. Step motion on crystal surfaces. ii. Journal of Applied Physics, 40(2):614-618, 1969.

[9] Jacques G. Amar. Effects of long-range interactions in metal epitaxial growth. Physical Review B, 67(16):165425, 2003. PRB.

[10] Matthew M. Hawkeye and Michael J. Brett. Glancing angle deposition: Fabrication, properties, and applications of micro- and nanostructured thin films. $J$. Vac. Sci. Technol. A, 25(5):1317, 2007.

[11] C. E. Botez, W. C. Elliott, P. F. Miceli, and P. W. Stephens. Vacancy formation in homoepitaxially grown ag films and its effect on surface morphology. Physical Review B, 66(7):075418, 2002.

[12] K. J. Caspersen and J. W. Evans. Metal homoepitaxial growth at very low temperatures:lattice-gas models with restricted downward funneling. Physical Review B, 64(7):075401, 2001. PRB.

[13] P. Ehrhart, K. H. Robrock, and H. R. Schober. page 60. Elsevier Science, Amsterdam, 1986.

[14] P. H. Dederichs. Diffuse scattering from defect clusters near bragg reflections. Physical Review B, 4(4):1041-1050, 1971. PRB. 
[15] Matthew M. Hawkeye and Michael J. Brett. Glancing angle deposition: Fabrication, properties, and applications of micro- and nanostructured thin films. $J$. Vac. Sci. Technol. A, 25(5):1317, 2007.

[16] S. Asgharizadeh, M. Sutton, K. Robbie, and T. Brown. X-ray reflectometry characterization of porous silicon films prepared by a glancing-angle deposition method. Physical Review B, 79(12):125405, 2009. PRB.

[17] K. Robbie, J. C. Sit, and M. J. Brett. Advanced techniques for glancing angle deposition. J. Vac. Sci. Technol. A B, 16(3):1115-1122, 1998.

[18] L. G. Parratt. Surface studies of solids by total reflection of x-rays. Physical Review, 95(2):359, 1954. Copyright (C) 2011 The American Physical Society Please report any problems to prola@aps.org PR.

[19] John F. Ankner and Charles F. Majkrzak. Subsurface profile refinement for neutron specular reflectivity (invited paper). S.P.I.E. Conference Proceedings, 1738, 1992.

[20] P. A. Kienzle, K. V. ODonovan, J. F. Ankner, N. F. Berk, and C. F. Majkrzak. Reflpak - mlayer. http://www.ncnr.nist.gov/reflpak, 2000-2006.

[21] unpublished in-situ work.

[22] P. F. Miceli. page 87. IOP, Bristol, 1993.

[23] I. K. Robinson, W. K. Waskiewicz, P. H. Fuoss, J. B. Stark, and P. A. Bennett. X-ray diffraction evidence of adatoms in the si(111)77 reconstructed surface. Physical Review B, 33(10):7013-7016, 1986. 
[24] C. E. Botez, W. C. Elliott, K. Li, E. D. Lu, P. F. Miceli, E. H. Conrad, and P. W. Stephens. Specular reflectivity from pyramidal surface morphologies. Physica B, 336(1):130-135, 2003. 


\section{VITA}

Shawn T. Hayden Sr. was born in Champaign, Illinois. He grew up in Abilene, Texas, where he received his B.S. in Physics at Abilene Christian University. After

completing his Ph.D in Physics at the University of Missouri under his advisor Paul F. Miceli, he will work as a Process Engineer for Intel, Hillsboro, Oregon.

He married his beautiful wife, Dora, in Abilene, Texas, and has 4 wonderful children, Crystal, Katie, Shawn Jr., and James. 